\title{
A Survey of Methods for 3D Histology Reconstruction
}

\author{
Jonas Pichat ${ }^{\mathrm{a}, *}$, Juan Eugenio Iglesias ${ }^{\mathrm{a}}$, Tarek Yousry ${ }^{\mathrm{b}}$, Sébastien Ourselin $^{\mathrm{a}, \mathrm{c}}$, Marc Modat $^{\mathrm{a}}$ \\ ${ }^{a}$ Translational Imaging Group, CMIC, University College London, UK \\ ${ }^{b}$ Department of Brain Repair and Rehabilitation, UCL Institute of Neurology, UK \\ ${ }^{c}$ Wellcome / EPSRC Centre for Interventional and Surgical Sciences, UCL, UK
}

\begin{abstract}
Histology permits the observation of otherwise invisible structures of the internal topography of a specimen. Although it enables the investigation of tissues at a cellular level, it is invasive and breaks topology due to cutting. Three-dimensional (3D) reconstruction was thus introduced to overcome the limitations of single-section studies in a dimensional scope. 3D reconstruction finds its roots in embryology, where it enabled the visualisation of spatial relationships of developing systems and organs, and extended to biomedicine, where the observation of individual, stained sections provided only partial understanding of normal and abnormal tissues. However, despite bringing visual awareness, recovering realistic reconstructions is elusive without prior knowledge about the tissue shape.

3D medical imaging made such structural ground truths available. In addition, combining non-invasive imaging with histology unveiled invaluable opportunities to relate macroscopic information to the underlying microscopic properties of tissues through the establishment of spatial correspondences; image registration is one technique that permits the automation of such a process and we describe reconstruction methods that rely on it. It is thereby possible to recover the original topology of histology and lost relationships, gain insight into what affects the signals used to construct medical images (and characterise them), or build high resolution anatomical atlases.

This paper reviews almost three decades of methods for 3D histology reconstruction from serial sections, used in the study of many different types of tissue. We first summarise the process that produces digitised sections from a tissue specimen in order to understand the peculiarity of the data, the associated artefacts and some possible ways to minimise them. We then describe methods for 3D histology reconstruction with and without the help of 3D medical imaging, along with methods of validation and some applications. We finally attempt to identify the trends and challenges that the field is facing, many of which are derived from the cross-disciplinary nature of the problem as it involves the collaboration between physicists, histopathologists, computer scientists and physicians.
\end{abstract}

Keywords: Histology, 3D Reconstruction, Registration, Medical Imaging

\section{Introduction}

Histology is concerned with the various methods of microscopic examination of a thin tissue section (or slice) (Culling, 2013), most commonly sampled from a specimen post mortem or from a biopsy. Cutting through a specimen reveals its internal topography and staining the sections permits the observation of complex differentiated structures. Then, the digitisation of histological sections (referred to as digital pathology) makes high-resolution microscope sections available for image computing and machine learning algorithms. These allow for disease detection, characterisation and prediction so as to complement the opinion of the pathologist (Madabhushi and Lee, 2016) and constitute the field of histopathological image analysis (Gurcan et al., 2009).

When willing to extend such examinations to the third dimension, one faces the following problem: starting from (a series of) 2D samples, how to regain information of the structure in 3D? Volume slicing breaks the spatial relations between structures and creates discontinuities which hamper mental representations in 3D and thereby, a full

\footnotetext{
${ }^{*}$ Corresponding author:

Email address: jonas.pichat.130ucl.ac.uk (Jonas Pichat)
} 


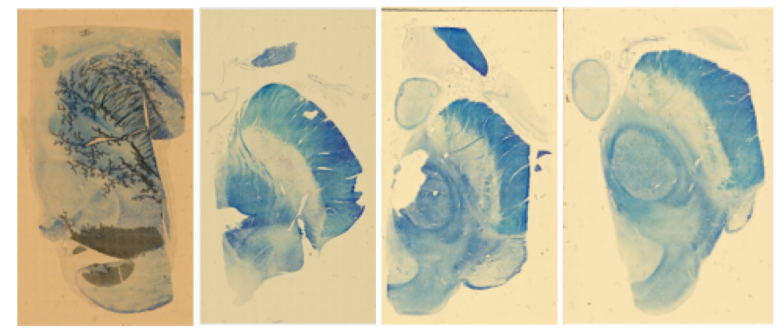

Figure 1: Artefacts related to the preparation of tissue sections (wax-embedded here). Those include (but are not limited to) intensity inhomogeneities, stain diffusion, tears, missing pieces, debris, air bubbles, various orientations and locations on glass slides, and spatial distortions.

understanding of the anatomy. In this respect, Gagnier and Shipley (2013) described the complexity in determining the progression of features into a volume by solely relying on a single face.

In addition, structures are independently altered due to the histological preparation itself (Fig. 1). This may cause anatomically different structures to look similar in microscope slides and conversely, slicing may cause one same structure to have different views if not consistent. Other changes have to do with objects that may disappear or become highly salient from one section to another due to staining variability.

Although humans can represent and mentally transform the shapes of objects very well, this ability worsens when structures are interconnected within a dense and complicated environment, or subject to complex transformations (Atit et al., 2013; Frick et al., 2014). Reconstructing histological volumes from serial 2D sections thus seems natural in order to (re)gain knowledge about spatial environments in 3D, while accessing microscopic information about tissues. In this regard, the Swiss anatomist Wilhelm His Sr. (1831-1904) best explained that "just looking through sections does not enable one to build three-dimensional images in the mind and those who wish to grasp anatomical structures must actively engage in working through a reconstruction, reproducing the relationships they wish to understand" (Hopwood, 1999).

When using histology alone, reconstruction algorithms aim to restore continuity and usually exploit the fact that the biological specimen's shape changes smoothly across sections. In other words, a set of slices is assumed available, with appropriate spacing (i.e., not too sparse) so that one can define a (reconstructed) volume. Such algorithms provide a representation of structures and their environment in three dimensions, although one needs to bear in mind that the original shape is unattainable without prior knowledge. For illustration purposes, Malandain et al. (2004) pointed out that if a banana is sliced, an ellipsoid will be reconstructed through pairwise alignment of adjacent slices, rather than the original fruit. This is called the "banana effect" or " $\mathrm{z}$-shift".

The most direct way of recovering volumes from sets of 2D serial histological sections is by optimising the spatial alignment of every pair of adjacent images using registration techniques. Image registration permits the automation of this transformation process, and allows to redefine "visual closeness" as the optimisation of a certain cost function. It also accounts for the complex transformations that affect hitological sections individually and grants higher reproducibility with less or no human effort. Composing the transformations from every image to a reference image completes the process - the reference section being chosen for its high contrast, its small amount of artefacts, and preferably but not necessarily its location in the middle of the stack. A consequence is that any registration error impacts the final reconstruction by propagation due to the sequential nature of the procedure. Methods have therefore been developed to minimise these effects by looking at neighbourhoods rather than single slices in order to smooth those errors out; attention has also been directed toward preprocessing the images of tissue sections owing to their highly variable quality.

A remedy to the incorrectness of histology reconstruction is the use of 3D medical images, such as magnetic resonance imaging (MRI). By providing structural ground truth, they refine the space of solutions-although registration itself remains an ill-posed problem. Careful use of registration techniques can produce histology reconstructions closer to reality, establish more accurate correspondences across modalities and thereby contribute to more sound data analyses. Two cases occur: (i) only a single (or too few) histological sections are available (like for biopsies), whereby a volume reconstruction is meaningless and one solely aims to identify the corresponding (resampled) MRI plane in order to deform histology correctly. In that case, one cannot rely on the greater supports that volumes offer, and such a 
situation calls for careful initialisation and 2D-3D registration methods (Ferrante and Paragios, 2017); (ii) a sufficient number of histological sections is available (i.e., the set spans several MRI slices) and one can thus manipulate volumes, globally bring them into spatial alignment, and non-linearly register each slice with its corresponding (resampled) MRI plane. In the process of relating in vivo to post mortem, it is not uncommon to use intermediate modalities (Fig. 2), such as blockface photographs (pictures of the tissue face taken prior to cutting), so as to keep track on the deformations that the tissue undergoes during its changes of state; or take advantage of needles, which allow for straightforward extraction and matching of landmarks in both modalities.

Besides providing structural ground truth, 3D medical imaging constitutes an invaluable source for accurate, noninvasive study of biological structures and their functions. Relative to histology, Fischl (2013) listed three advantages: the possibility of (i) imaging the exact same tissue with multiple contrasts (e.g., T1 or T2w MRI, MTR, etc.); (ii) imaging large samples (e.g., whole-brain or whole-hemisphere) with much less effort than e.g., whole-brain or prostate whole-mount histology; (iii) preserving the geometry of the sample and avoiding irreversible damage and distortions induced by processing, cutting, mounting and staining during the histological preparation.

With respect to resolution, MR imaging is outperformed by histology $(<1 \mu \mathrm{m})$. In addition, for many pathological disorders, there is still no no sequence acquisition that allows imaging to be a full substitute for histology. This is due to the poorly understood relationships between histological and magnetic properties of tissues. Directly predicting the imaging appearance of a histological signature is therefore extremely complex. Practically, this results in that different pathologies can share a common imaging phenotype (Gore, 2015). For example, Filippi et al. (2012) noted that in proton density, FLAIR and T2w MRI scans, Multiple Sclerosis (MS) lesions appeared as non-specific focal areas of signal increase and, therefore, resembled many other types of pathology. This makes it difficult to differentiate them with imaging only. Additionally, some cortical MS lesions can still be missed with conventional MRI sequences (Seewann et al., 2012). Direct comparisons with histology helps interpret images better and derive more information. They may also help in correcting or adjusting existing imaging protocols in order to optimally visualise pathological markers (e.g., lesions in the grey matter of patients with MS).

One of the many benefits of combining histology and medical imaging is to confirm non-invasive measures with baseline information on the actual properties of tissues (Annese, 2012). By combining 3D medical imaging with digital pathology, it is possible to simultaneously obtain the rich structural information of the former and the chemical and cellular information of the latter, which may allow for more complete characterisation and understanding of $e$.g., diseases (Mori, 2016). One can also derive more accurate segmentations of architectonic boundaries to be used in the creation of atlases (Ding et al., 2016; Oh et al., 2014; Amunts et al., 2013; Hawrylycz et al., 2012; Yushkevich et al., 2009) as well as brain mapping (Amunts and Zilles, 2015). Such undertakings are intended to eventually bridge the gap between in vivo and post mortem studies.

Currently, direct overall visual comparison is considered acceptable to assess the correlation between histopathological findings and imaging observations. On that matter though, it was recently mentioned in the context of prostate cancer assessment that due to variations in imaging technologies, contouring procedures and data analyses, available volume correlation studies had yielded conflicting results (Priester et al., 2016). Such contradictions were explained by the worrying observation that nearly all prior attempts to define MRI/pathological relationships had relied on imprecise techniques such as manual registration, volume approximation, and 2D measurements. Following the same line of thought - two decades before-correlation was proved to be optimised when the alignment between data had first been carefully taken care of by use of a combination of linear and non-linear transformations (Mazziotta et al., 1995). In other words, ensuring the comparison of like with like is of utmost importance (Madabhushi and Lee, 2016). In this paper, we describe methodologies which relied on (automatic) image registration techniques.

The objective of this paper is to survey the past 30 years of literature on $3 \mathrm{D}$ histology reconstruction. The paper is structured according to the multidisciplinary nature of the problem. $\S 2$ and 3 explain the preparation of histological slices, list artefacts associated with every step of the process and cover preprocessing methods in order to best cope with image deteriorations. $\$ 4$ proposes a classification of methods for 3D histology reconstruction relying solely on $2 \mathrm{D}$ serial sections and $\S 5$ describes pipelines that aim to combine histological and volumetric medical imaging information. $\S 6$ presents approaches used to validate the correctness of reconstructions-with or without the help of external information-and $\S 7$ enumerates the clinical applications of such techniques. Finally, $\S 8$ returns on a few methodological points, discusses some of the remaining challenges in the field and highlights the importance of cross-disciplinary knowledge in solving a biological question. 


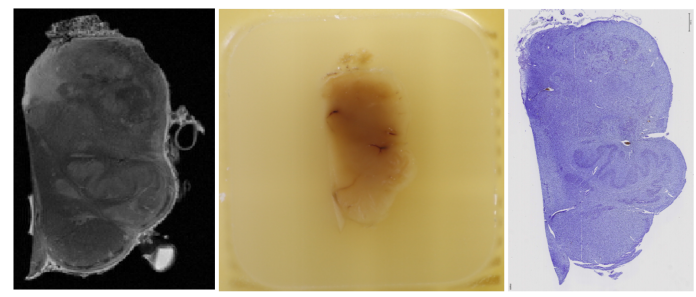

Figure 2: Modalities that may be involved in the registration process. From left to right, a screenshot of a T2w ex vivo slice $\left(0.1 \times 0.1 \times 0.4 \mathrm{~mm}^{3}\right)$, a visually corresponding blockface photograph (tissue surrounded by wax) and a Nissl-stained histological section.

\section{From fresh tissue to digital pathology}

A pathologist receiving fresh tissue has three options: keeping it fresh, stabilising it in a fixative, or freezing it. Biological tissue is too soft for direct sectioning (although a vibrating blade might work), so it is most commonly either embedded in a hardening material and sectioned using a microtome, or frozen and sectioned in a cryostat (a microtome inside a freezer). Sections are then mounted on glass slides and stained before being observed under the microscope by the histopathologist, and/or digitised using flatbed scanners (Dubois et al., 2007) for image processing and analysis.

We first briefly describe the two most common processes to obtain sections, namely formalin-fixed paraffinembedded (FFPE) sections (\$2.1) — henceforth referred to as paraffin sections—and frozen sections (\$2.2). Further details can be found in the thorough presentation of histological techniques by Bancroft and Gamble (2008). Then, we briefly present several types of microscopy examinations and the process of digitisation (\$2.3). Finally, we highlight the most common artefacts for both types of sections (\$2.4).

\subsection{Paraffin sections}

FFPE tissue sections stained with hematoxylin and eosin (H\&E) are the gold standard (Buesa, 2007) as they provide with generic information in very little time and cost (Rosai, 2007). Their widespread use also relates to the familiarity histopathologists have with the method: the artefact it produces at any stage during tissue handling and processing is recognisable and well-documented. In contrast, observing new patterns with other dyes requires time and training (Bancroft and Gamble, 2008). The above-mentioned artefact is to be taken in the sense that it refers to an altered state of the tissue and its structures (relatively to its living state) i.e., the structures it exhibits are not naturally present in the living state of the tissue but are rather the product of a series of preparation steps (Hardy, 1899); throughout the rest of the paper, the definition of artefact is narrowed down to (image) degradations. Knowledge of the steps relative to tissue preparation and diverse staining patterns is not only essential for diagnosis and risk assessment-and this is still an active area of research (Kakar et al., 2015) - but also for all subsequent image analysis steps. In the following, we briefly describe the different stages of FFPE sections preparation.

Fixation. It is the most important step when performing histological specimen preparation (Rolls, 2012). Fixation is critical for several reasons: (i) it prevents the tissue from autolysis; (ii) it keeps the tissue close to its living state, without loss of arrangement; (iii) it minimises changes in shape or volume in subsequent procedures and (iv) it yields clear staining of sections. Formaldehydes, such as formalin — which is the most common of all-are routinely used for chemical fixation, such as in Yang et al. (2013); Chen et al. (2003); Bürgel et al. (1999); Weninger et al. (1998); Schormann and Zilles (1998); Streicher et al. (1997). Among others, glutaraldehydes may be used (Baheerathan et al., 1998).

Tissue processing. Since most fixatives are water-based and thus not miscible with wax, the tissue must be processed to enable impregnation. This process follows three steps: (i) dehydration: it removes water by immersion in gradients of alcohol; (ii) clearing: it replaces the dehydrating fluid with a wax solvent (the wax solvent has the effect of raising the refractive index of the tissue, making it appear clear, hence the name). Note that long-term clearing creates distortions. Xylene is routinely used for short schedules and blocks less than $4 \mathrm{~mm}$ thick. Among others, toluene is also used and has similar properties; (iii) impregnation: it replaces the clearing agent with the embedding medium. 
Embedding. The specimen infiltrated with wax is put in a mould covered with liquefied wax and topped with a cassette. The specimen should lay flat at the bottom of the mould as its orientation conditions the plane of sectioning (an important consideration when flatness is assumed for the comparison with clinical imaging). The ensemble then cools on a cold plate and makes a solid block for microtomy (blocks may also be stored at room temperature for decades, which forms an important archive in retrospective analyses). Paraffin was used for example, in Axer et al. (2011); Alic et al. (2011); Bajcsy et al. (2006); Breen et al. (2005b); Schormann et al. (1995). Celloidin, more difficult to remove, was used in Li et al. (2009); Gefen et al. (2008); Beare et al. (2008).

Cutting (or microtomy). It is performed with a microtome, to which the cassette with the wax-embedded tissue block is clamped. It begins with "trimming", which consists of removing the surplus of wax until a full section of tissue is available. It requires great care since tissue of diagnostic importance may be removed or the block surface damaged. Cutting is then processed at a certain thickness and the quality of the resulting sections depends upon several factors such as the knife angle, blade quality, speed of sectioning etc., as well as all the previous preparation steps. Thin sections (1-20 $\mu \mathrm{m})$ were cut in Samavati et al. (2011); Zhan et al. (2007); Burton et al. (2006). Thick sections (>20 $\mu \mathrm{m})$ were cut in Jiang et al. (2013); Osechinskiy and Kruggel (2010); Mazaheri et al. (2010); Singh et al. (2008).

Floating, drying. The thin sections are picked up from the microtome and put in a flotation bath, filled with warm water in order to flatten. Then, they are collected on a glass slide and dried.

Staining, cover-slipping. It is the process of colouring and differentiating certain structures in the tissue. H\&E stain is the most common stain in histopathology laboratories. It was used for instance in Le Nobin et al. (2015); Nir et al. (2014); Gibson et al. (2012); Ward et al. (2012); Arganda-Carreras et al. (2010); Ou and Davatzikos (2009); Meyer et al. (2006). H\&E method shows a wide range of normal and abnormal cell and tissue components and is easy to perform using either paraffin or frozen sections. Other popular stains include Cresyl violet (Nissl staining), as used in Adler et al. (2014); Yang et al. (2012); Mailly et al. (2010); Johnson et al. (2010); Chakravarty et al. (2006); Ali and Cohen (1998), and methylene blue (Annese et al., 2006) for nervous tissue sections, silver and gold methods to demonstrate e.g., cell processes in neurones, toluidine blue (Handschuh et al., 2010) to stain acidic components, Masson's trichrome (Song et al., 2013) to stain connective tissue and Alcian blue (Magee et al., 2015) to stain certain types of mucin. If immunohistochemical staining is to be performed, it requires antigen retrieval (heat- or enzyme-enduced) due to loss of antigenicity during fixation (Shi et al., 1991). Immunohistochemistry (IHC) was performed in Capek et al. (2009); Groen et al. (2010).

After the slice has been stained, it is cover-slipped: a smaller sheet of glass covers the tissue mounted on the glass slide. This creates even thickness for viewing and prevents the microscope lens from touching the tissue. The slide can then be observed under the microscope and/or digitised.

\subsection{Frozen sections}

Frozen sections are quicker to produce than paraffin sections but it is a very demanding process: good section quality (in terms of preservation of tissue morphology) is achieved through great care and expertise (Peters, 2003). Although there are conflicting reports about how much freezing may degrade cell morphology and reduce the readability of histological specimens, rapid freezing is known for limiting ice crystal formation and minimising morphological damage. Among disadvantages, it is harder to make the tissue lay flat; frozen sections are also more difficult to cut than paraffin sections and inconvenient to store. The main advantages of using them are the shortcuts in the process (e.g., no dehydration is needed), and their better preservation of antigens for immunohistochemistry. They were used in Annese et al. (2014); Stille et al. (2013); Annese (2012); Choe et al. (2011); Palm et al. (2010, 2008); Dauguet et al. (2007c). The different stages of frozen sections preparation consist of:

Cryo-protection/embedding. The limiting factor involved in cryosectioning is the cutting consistency of the block and the freezing damages from ice crystals. Thus, the tissue may require cryoprotection to make it less brittle (Barthel and Raymond, 1990). Cryoprotecting the tissue is not necessary and consists of fixation (formaldehyde), rinsing and infiltration in increasing series of sucrose solutions. The addition of sucrose provides a smoother cutting block and minimises freezing artefacts. It also happens that sections are prepared from fresh, rapid-frozen tissue but cutting can be incredibly hard without any fixation. Then, optimal cutting temperature (OCT) compound is used to embed the 
tissue prior to frozen sectioning. OCT helps conduct heat away from the specimen during freezing, protects the tissue from drying during storage, and supports the tissue during sectioning.

Rapid freezing (or flash/snap freezing). Once embedded in a particular orientation e.g., face-up, the tissue sample needs to be rapidly frozen to minimise freezing artefacts resulting from ice crystal formation as water freezes in the tissue (Peters, 2010). One method is to use dry ice ( $-70^{\circ}$ Celsius) on its own. It is simple and safe but creates freezing artefacts that break cell membranes. An alternative is the immersion of the sample in a freezing medium, like a mixture of dry ice and 2-methyl butane (isopentane), which achieves very rapid freezing. Note that direct freezing would cause the tissues or blocks to crack, which would make them very difficult to cut. Tissues with fat often produce poor quality sections since fat freezes at lower temperatures and thus remains too soft to cut; further decreasing temperature may weaken the sample and cause cracks. Tissues with substantial water content, like the brain, often yield ice crystals during the freezing in the cryostat and result in e.g., non-representative architecture of tumour growth or inflammatory infiltrate (Taxy, 2009). Snap freezing with liquid nitrogen is often employed to mitigate these artefacts. The frozen tissue can then be stored in a $-80^{\circ}$ Celsius freezer for future cutting.

Cutting. This is similar to paraffin-embedded sections except it is performed in a cryostat. It also starts with trimming of the block. Frozen sections are usually cut between $3-10 \mu \mathrm{m}$ thick $(5 \mu \mathrm{m}$ thick sections provide adequate morphology). Ultra-thin sections $(<1 \mu \mathrm{m})$ were cut in Yushkevich et al. (2006) $(0.25 \mu \mathrm{m})$. Thin sections $(1-20 \mu \mathrm{m})$ were cut in Dubois et al. (2007) and Humm et al. (2003). Thick sections (>20 $\mathrm{m}$ ) were cut in Palm et al. (2010) and Malandain et al. (2004).

Retrieving, drying. Retrieving is the process of picking up the cut frozen section and putting it on a glass microscope slide. Tissue sections can be either picked up from the cryostat stage or from the block directly. From the time the tissue section touches a warm slide, it starts to develop a drying artefact. Air drying frozen section slides will however allow the sections to better adhere to the slide as complex staining procedures cause greater tendency for the tissue to come off the slide during staining.

Fixation. Sections of fresh frozen tissue should be fixed immediately unless they are going to be stored for future study. A standard histology fixative: $4 \%$ neutral buffered formalin, is the most suitable fixative for frozen sections. Sections of fresh frozen tissue will rapidly dry if exposed to warm air, and this will result in cellular artefact.

Staining. Slides prepared by frozen section technique can be successfully stained by many of the staining procedures used for routine paraffin embedded tissues. For example Nissl-stained sections were used in Yushkevich et al. (2006); Yelnik et al. (2007); Dubois et al. (2007) and H\&E stained sections were used in Humm et al. (2003). Frozen sections are usually preferred for immunohistochemical staining due preserved antigenicity. This a specific type of stain, in which a primary antibody is used to bind specifically to a particular protein for the purpose of detecting and measuring it. Then, a secondary antibody (which carries a colorimetric or fluorescent detection tag) is used to bind to the primary antibody and reveal its bounding location. IHC was performed in Seeley et al. (2014); Stille et al. (2013); Lockwood-Estrin et al. (2012); Lebenberg et al. (2010).

\subsection{Microscopy and digitisation}

Major types of light (or optical) microscopy include brightfield (Wang et al., 2014), phase contrast, fluorescence (Dauguet et al., 2007c) and confocal (Wang et al., 2015). Electron microscopy encompasses transmission electron microscopy (Dauguet et al., 2007a) and scanning electron microscopy, the latter being mainly used in the context of serial blockface imaging (Mikula and Denk, 2015; Denk and Horstmann, 2004). The preparation of tissue specimens for light microscopy follows the steps from $\$ 2.1$ and 2.2. The preparation of tissues for transmission electron microscopy is described in Graham and Orenstein (2007).

As for immunocytochemistry and immunohistochemistry (Yelnik et al., 2007), the reaction of antibody with antigen in can be examined and photographed with a fluorescence microscope. Histochemical and cytochemical procedures (based on e.g., specific binding of a dye, a fluorescent dye-labeled antibody or enzymatic activity), can be used with both light microscopic and electron microscopic preparations. Light and electron microscopes produce high resolution micrographs (orders of magnitude of $0.1 \mu \mathrm{m}$ and $1 \mathrm{~nm}$ respectively). 
Autoradiography — or to be rigorous, radioautography (Belanger and LeBlond, 1946), can be observed with both light and transmission electron microscopes and reflects the rate of the energy consumption required to support cellular activity. It is quantified using tracers of glucose metabolism incorporated by living cells and tissues. They generate a labelled product allowing for example, to measure circulating glucose in the blood or radioactivity concentrations. The specimen is then killed and a sample is processed for histology and sectioned. Sections are placed against an $\mathrm{X}$-ray film to produce autoradiographs. The exact 3D localisation of the radiation source is however unknown and thus requires the reconstruction of autoradiographic volumes (Schubert et al., 2016). Reconstruction is also a pre-requisite for comparison against other three-dimensional modalities such as functional imaging.

Although in the context of multimodal image registration, computer scientists usually work with histological images at low resolution, similar to that of a clinical image - most high-resolution detail in histology is biological noise for the purpose of registration-digital pathology should allow the histopathologist to scroll through any level of details of a "virtual" microscopic slide for its examination at any time and anywhere (i.e., not under a microscope), should it be on its own, against another histological section or a medical image plane. This process of digitisation is fundamental (Ghaznavi et al., 2013) and brings together the fields of virtual microscopy, digital whole slide imaging and telepathology (Weinstein et al., 2009).

\subsection{Artefacts}

In histology, an artefact is the result of the alteration of a tissue from its living state, caused by the very process of dying and the histological preparation. Artefacts affect different structures from one same tissue section independently, and one same structure in adjacent tissue sections differently. Artefacts may compromise both image analysis for accurate diagnosis and image registration for precise alignment. One challenge is to be able to identify artefacts and not confuse them with normal tissue components or pathological changes. This means understanding the causes of such deteriorations as well as their expression in order to provide relevant corrections.

Whether paraffin or frozen sections are used, some artefacts have similar characteristics despite having different causes. This makes some preprocessing methods applicable to both types of sections. An exhaustive list of artefacts encountered in paraffin sections, along with ways to minimise them is available in Rolls et al. (2008) and the most common ones are:

Loss of detail. In paraffin sections, delayed fixation may cause poorly defined nuclei and imprecise cytoplasmic details. Improper draining of sections before drying may lead to out-of-focus areas, and imperfect dehydration before clearing, which leaves small water droplets in the tissue, may cause opaque areas. Similarly to frozen sections, drying (which starts as soon as the tissue is in contact with a warm glass slide) may blur nuclear details and cytoplasmic borders (due to the leakage of fluids from the cytoplasm), and a loss of contrast. Drying artefacts are described as cells melting and spreading on the slide by Peters (2010).

Changes in morphology. In paraffin sections, the use of an overheated forceps (beyond the melting point of wax) can cause local heat damage and changes in morphology of structures in the area surrounding the contact point. In frozen sections, drying may cause enlargement of cells and nuclei.

Uneven staining. In paraffin sections, it may come from incomplete fixation of the specimen (which leads to zonal fixation), incomplete slide dewaxing (which results in slides containing patches of residual wax and produces unstained, or unevenly stained areas) and excessive heat in the slide drier. Approximate timing as well as different storage conditions also produce inconsistent results across sections. Poor quality formalin results in a "formalin pigment" formation in sections by reaction with haemoglobin, leading to unwanted colouration. As for frozen sections, issues may arise due to over-agitation of loosely adherent tissue in the staining solution.

Folds and wrinkles. In paraffin sections, they may be due to poor fixation and/or processing, too large a clearance angle of the microtome, too thin sections, low temperature of the flotation bath (which may not allow sections to flatten properly) or mechanical damages (when attempting to remove a fold in the section with a brush). As for frozen sections, the tissue can fold, stretch or tear if one is too rough during retrieving. 
Cracks and holes. In paraffin sections, they may happen due to over-processing (which makes the tissue very brittle), under-processing (which makes the tissue poorly supported and therefore fragmented), flotation on a water bath that is too warm, prolonged drying at too high a temperature, too quick sectioning, insufficient clearance angle or a damaged cutting blade during microtomy. As for frozen sections, freezing blocks (instead of cooling them down) can make them crack during cutting. Another challenge is faced with large blocks of tissue, such as whole organs: liquid nitrogen will freeze faster and create a shell around the exterior of the tissue. Then, the organ is likely to crack when the interior expands due to slower freezing.

Contaminants. In paraffin sections, this may happen when the water from the flotation bath is not replaced regularly, which favours contaminants that may end up on the slide under the section. Dust, organisms and other contaminants on the glass slide can also spoil otherwise good sections.

Compression and distortion. In paraffin sections, they may be due to under-processing (which results in the shrinkage of the specimen); inappropriate size of the container compared to the size of the specimen (which means using an insufficient amount of fixative or squashing the specimen inside); rough handling; poor quality embedding wax (which produces blocks that are difficult to cut); suboptimal knife tilt angle during microtomy and wrong blade type; delay before cutting the final sections of a block (which makes the block warmer); and overheated flotation bath and sections left too long in it (which cause over-expansion). It is also important to be aware that paraffin sections are unlikely to be of even thickness as the first couple of sections are the widest (due to the thermal expansion of the block during the first passes across the knife) and the least compressed; however as the block warms the sections get narrower and more compressed. As for frozen sections, compression and distortion will most likely result from ice crystal formation-the more water a tissue contains, the more chances artefacts will occur. As water freezes, the expansion of ice crystals compresses cellular tissues (compression artefacts) and distort histopathological correlations. They usually have the appearance of bubbles (ice crystals "bubbles" artefacts). The knife used in cryosectioning can also create cutting artefacts (shearing of the tissue).

In the end, artefacts are unavoidable but also surmountable as pathologists learn to read around them. However, it is very important to try to minimise their impact on subsequent steps, which heavily rely on the tissue quality: for example, sections with cracks and holes often have to be manually discarded because they cannot be registered. Artefacts hamper image computing methods by reducing comparability between supposedly similar structures within or across modalities. For this reason, preprocessing methods have been developed to account for their presence in images.

\section{Preprocessing of digital pathology}

Among the artefacts resulting from histological preparation, loss of detail and changes in morphology burden image analysis. Not much can be done about them as content is hardly retrievable from lost or corrupted information without any prior knowledge. When due to scanning, though (local poor focusing can cause blurred regions in images), loss of detail is surmountable but at the cost of time-consuming review by the scanner operator. In the context of whole slide imaging, Lopez et al. (2013) automatically identified tiles that required additional focus points. Specifically, they compared the ability of several features in discriminating between blurred and sharp regions of images and showed that the Haralick contrasts and gradient-based features best performed at this task. Compression and distortion are usually taken care of by the process of registration. Regarding other artefacts such as: inhomogeneous intensity distributions within and across slices; folding and crumpling; cracks and holes, dedicated preprocessing methods are presented in the following paragraphs.

Inhomogeneous slices appearance. Ideally, the absolute colour of a slide reveals the biological component that a pathologist wishes to retrieve. For example, in the case of $\mathrm{H} \& \mathrm{E}$, the colour value quantifies the amount of nucleic acids (blue-purple) hematoxylin has bound to, and the amount of proteins (in pink) Eosin has bound to. However, for the reasons listed in \$2.4-and/or because of the microscope and the camera used for imaging (Yagi and Gilbertson, 2005) - slides exhibit different colours. Improved feature classification, segmentation and visualisation require the reduction of these variations as well as some sort of standardisation of the imaging protocols (Badano et al., 2015). This calls for transforming the appearance of a source image into that of a target image preferred by an expert. 
In general, histology reconstruction methods use greyscale images for intensity standardisation (or the channel that provides the best contrast in an RGB image). Most techniques are based on histogram matching (Gonzalez and Woods, 2002). One representative method, used for example in Yelnik et al. (2007) and Alegro et al. (2016), was proposed by Malandain and Bardinet (2003). First, continuous probability density functions from the discrete intensity histograms of two input slices were computed using Parzen windowing - a Gaussian kernel was also used in Ceritoglu et al. (2010) and Casero et al. (2017). Then, Malandain et al. estimated the optimal affine intensity transformation between them (though higher order polynomial fits may be used). This type of method can be applied in different ways and the reference slice is usually picked for its relative smooth intensity variation of staining and high-contrasted structures (Gaffling et al., 2009; Yang et al., 2013). Adler et al. (2014) optimised the parameters of a global affine intensity transformation using white and grey matter masked images jointly. The central slice was taken as a reference. Yang et al. (2012) used histogram equalisation, in which case a flat histogram is implicitly taken as reference for matching. Equalisation is however not robust because it is very sensitive to outliers (the extremal values of the intensity spectrum) (Malandain and Bardinet, 2003).

Attempts at decreasing the bias introduced by selecting a single reference slice have been proposed: Li et al. (2009) applied to each slice a transformation that was a weighted sum of transformations from that image to a set of reference images (experimentally, one every 30 slices). Weights were based on its distance to the corresponding references. Chakravarty et al. (2003) used least trimmed square (LTS) to calculate the coefficients of two polynomials of order three that mapped the intensities of the current slice to the previous and the next one. The coefficients of the two polynomials were then averaged and applied to the single slice. An extension consisted of adding an extra step that accounted for local variations: the same averaging process (though restricted to linear mapping) was applied to patches of every slice (Chakravarty et al., 2006). This approach however depends on where it starts in the stack. Pichat et al. (2015) computed an unbiased average intensity profile to which the intensity distributions of all slices were matched.

Should it be using a single, a set of, or an average reference distribution, normalisation always depends on the set of available histological slices. Hence, the purpose of standardising slices appearance is, in general, more to bring visual consistency and help with subsequent segmentation and classification tasks, than being representative of tissue behaviours relative to staining.

The idea of computing a standard histogram allows for a standardisation that is not "stack-specific". This was proposed by Nyul and Udupa (1999) within the context of medical imaging, where a standard histogram was computed from a training dataset made of images coming from several acquisition protocols. A similar principle was used by Bagci and Bai (2010). Dauguet et al. (2004) followed the same effort although standard values of each class of tissues had to be user-defined.

Within the field of histopathological image analysis, the importance of colour consistency has long been known and is an active research topic: computational methods, referred to as colour normalisation, have been developed to cope with inter-slice colour variations. Two ways of addressing the problem stand out: (i) colour modification methods represent the mathematical transformations applied to the source images to match the characteristics of a target image (Braumann et al., 2005) - they are similar to previously described intensity standardisation methods for grey-scale images; (ii) colour separation (or deconvolution) methods, concerned with first extracting the main components (i.e., the stains) constituting the original image (relying on the manual delineation of regions of interest, non-negative matrix factorizations, plane fitting in the optical density domain or other colour models), then normalising them individually and finally recombining them, such as in Macenko et al. (2009); Magee et al. (2009); Khan et al. (2014); Vicory et al. (2015); Vahadane et al. (2016); Bejnordi et al. (2016). These methods apply to sections stained with more than one dye, mostly H\&E stained images, and are still actively developed. Colour modification was introduced by Shirley (2001), who proposed to match the colour distribution of one image to that of a reference image by use of a linear transform in Lab colour space (a more perceptual colour model than RGB) so as to match the means and standard deviations of each colour channel in the two images in that colour space. This was applied to histological data in Wang et al. (2007). In order to account for scanner-induced variations, Bautista et al. (2014) proposed to use a colour-calibration slide made in-house to derive a colour correction matrix. Bautista and Yagi (2015) showed that it is possible to achieve consistent and accurate segmentations with simple classifiers by accounting for the staining conditions of the slides using dye amount tables.

Folds. They are defined as regions containing multiple layers of stained tissue. This results in regions with higher saturation and lower luminance. As such, Kothari et al. (2013) used the difference between colour saturation and 
luminance to detect them. They developed a model that adaptively finds the difference-value range of tissue folds in order to account for the high variability of colour saturation and intensity in different slides. Bautista et al. (2010) enhanced folds and limited the changes in hue by using the difference value between saturation and luminance as a shifting factor for pixel values. Palokangas et al. (2007) used $k$-means clustering on HSI (Hue, Saturation, Intensity)transformed images, although only saturation and intensity components were said to be discriminative enough. However, such clustering assumes that there are always folds in the images and the method relies on careful initialisation of cluster centres. Simple thresholds are said to be less effective because a tissue fold in a lightly stained image can look similar to e.g., a tumour in a darkly stained image (Kothari et al., 2013; Palokangas et al., 2007). Similarly to intensity/colour normalisation, fold detection and masking were shown to improve subsequent feature extraction and classification tasks.

The correction of folds may be one of the most difficult problem to solve here, mainly because of the interference of constituents caused by the overlap of different parts of the tissue. Although modelling of developable surfaces has been proposed in computer graphics (Solomon et al., 2012), the reconstruction of an unfolded tissue section is difficult as it supposes the separation of structures belonging to different overlapping bits of the tissue-the number of folded layers is also unknown a priori.

Tears (or cracks) and holes. Such artefacts are more frequently addressed than folds but their correction remains sparse. Cracks require, in general, manual delineation of the torn area (Breen et al., 2005b) as it is challenging to automatically tell whether a piece of tissue is missing or if the tissue has effectively opened up without loss of material. Yang et al. (2012) filled up the missing sections and missing parts and corrected folds using a procedure described in Qiu et al. (2009). Choe et al. (2011) proceeded with manual contouring of the torn area and filled it by repeating pixel values of the contour along the columns of that region. Such a process however makes a strong assumption about the horizontality of tears. A similar protocol was followed in Kindle et al. (2011). Breen et al. (2005a) used correspondences between landmarks to stitch the torn piece back: a first thin-plate splines (TPS) warping (Bookstein, 1989) was performed between histology and blockface photograph using manually defined sets of corresponding landmark points. Then, another set of landmarks was found at the borders of the torn piece of tissue in histology and in the intact corresponding piece of the blockface photo (both were overlaid to ease the process). Finally, a separate TPS warping was applied to register the torn piece of tissue back. Correspondences between sets of landmarks were found using the "live-wire" algorithm developed by Falcão et al. (1998) and Mortensen et al. (1992). One could also approach the problem of tear correction as jigsaw puzzle solving (Kong and Kimia, 2001; Paikin and Tal, 2015), although it has failed in Yigitsoy and Navab (2013) because these methods rely on borders and medical images usually have low signal as well as distorted boundaries. The tearing/cracking of thin sheets has been subject to extensive studies within the fields of statistical physics (Holmes and Crosby, 2010) or computer vision (Pfaff et al., 2014).

Masking. In order to discard various contaminants on the glass slide (they could have an influence in subsequent registration steps), the tissue is separated from the background. Thresholding is widely used (Nikou et al., 2003; Malandain et al., 2004; Lee et al., 2005; Dauguet et al., 2007b; Palm et al., 2010; Goubran et al., 2013; Stille et al., 2013) and it is usually complemented by mathematical morphology operations (Malandain and Bardinet, 2003; Dauguet et al., 2007b; Palm et al., 2010). Dubois et al. (2007) used iterative Gaussian smoothing of histograms for the automatic computation of thresholds: following Mangin et al. (1998), they tracked the positions of modes in the scale-space and the two modes that remained across most scales were picked as those representing background and tissue. Region growing was then applied in the histogram using previously computed upper and lower bounds. Masking was preformed with mathematical morphology (successive erosions using a priori knowledge of the tissue surface) and the largest connected component was extracted. Yushkevich et al. (2006) used active contour segmentation with region competition (Zhu and Yuille, 1996) followed by mathematical morphology to refine the masks: opening (which is less destructive than erosion alone but still removes isolated pixels) was performed and the largest connected component was kept as final mask. Level-sets were used with a dynamic speed function in Li et al. (2009), and in Uberti et al. (2009) based on Li et al. (2005). They incorporated higher level constraints obtained from prior knowledge and understanding of mouse brain anatomy. Palm et al. (2010) used $k$-means clustering on the "a" channel, after transformation from RGB to CIELab colour space, to segment tissue in blockface photographs. This was followed by a hole-filling algorithm. Adler et al. (2014) used Atropo (Avants et al., 2011), an $n$-class Markov random field segmentation software package for tissue foreground segmentation. They used three labels: grey matter, white matter and background-grey matter 
Table 1: Company/academic softwares and plugins for histology reconstruction from serial sections and their use in the literature.

\begin{tabular}{rlcll}
\hline softwares/plugins & institution & non-rigid warping & references & used in \\
\hline \hline TrakEM2 (ImageJ) & Uni of Zürich (CHE) & no & (Cardona et al., 2012) & (Chklovskii et al., 2010) \\
StackReg (ImageJ) & EPFL (CHE) & no & (Thevenaz et al., 1998) & (Micheva and Smith, 2007) \\
AutoAligner & Bitplane (CHE) & no & - & (Friedrich and Beutel, 2010) \\
Voloom & TU München (DEU) & yes & (Feuerstein et al., 2011) & (Fónyad et al., 2015) \\
BrainView & LIN (DEU) & yes & (Lohmann et al., 1998) & (Dubois et al., 2007) \\
Free-D & INRA (FRA) & yes & (Andrey and Maurin, 2005) & (Bonnet et al., 2013) \\
BrainVISA & CEA I BM (FRA) & yes & (Cointepas et al., 2001) & (Dubois et al., 2007) \\
AlignSlices (Amira) & FEI VSG (FRA) & no & - & (Andersson et al., 2008; Cornillie et al., 2008) \\
3DHISTECH & (HUN) & - & - & (Onozato et al., 2013) \\
poSSum & Nencki Institute (POL) & yes & (Majka and Wójcik, 2015) & (Majka and Wójcik, 2015) \\
ImageRegistration (ImageJ) & NTUST (TWN) & yes & (Wang et al., 2014) & (Wang et al., 2014) \\
BioVis3D & (URY) & - & - & (Dezsó et al., 2012) \\
HistoloZee & UPenn (USA) & yes & (Adler et al., 2014) & (Yushkevich et al., 2016) \\
Protomo & Florida SU (USA) & no & (Winkler, 2007) & (Singh et al., 2016) \\
Reconstruct & Boston Uni (USA) & yes & (Fiala, 2005) & (Mathiisen et al., 2010) \\
IMOD & Uni of Colorado (USA) & yes & (Kremer et al., 1996) & (Mishchenko, 2009) \\
ImageJ & NIH (USA) & no & (Schneider et al., 2012) & (Le Nobin et al., 2015) \\
NIH Image & NIH (USA) & no & (Rasband and Bright, 1995) & (Laissue et al., 1999)
\end{tabular}

and white matter labels were grouped into foreground tissue mask, from which the largest connected component was retained.

Vignetting (or shading). A common problem irrespective of the type of camera or microscopy, is uneven illumination of the scene being imaged (Leong et al., 2003). It occurs in most imaging sensors and results in images that are lighter near the optical centre and darker at the borders (i.e., a shading artefact). This effect is particularly evident when stitching images into a mosaic in order to increase the field of view of the microscope to obtain $e . g$., whole-slide images. Correction of uneven illumination in histological slices has borrowed ideas from intensity inhomogeneity correction in MRI (Sled et al., 1998; Vovk et al., 2007). The correction of vignetting was addressed by Peng et al. (2014) and Piccinini et al. (2013b), and the interested reader may also refer to Reyes-Aldasoro (2009); Yu (2004). In the histology reconstruction literature, shading correction was performed as preprocessing in Bürgel et al. (1999) using methods from Gonzalez (1987). Arganda-Carreras et al. (2010) developed a background correction algorithm based on a phantom (Fernandez-Gonzalez et al., 2004) that was used to correct the mosaic-like effect of the images caused by uneven illumination of the field of view of the microscope. Methods for compensation of such a light variability were also applied in Capek et al. (2009), further described in Čapek et al. (2006). Colour difference and optical degradation were accounted for in Hsu et al. (2008), by means of a Gaussian-like model and a wavelet-based image blending.

Stitching (or mosaicing). It is needed when the field of view of a classical microscope is too narrow to allow for the visualisation of the entire tissue. The section can either be physically cut into several pieces that are isolated in the image (see Chappelow et al. (2011b), or Ou and Davatzikos (2009), who simulated it), or spatial tiles can be obtained by moving the microscope stage (Capek et al., 2009). The latter protocol however introduces overlapping between adjacent fields of view. Overlap is recommended to account for field curvature-induced artefacts in the image and avoid loss of detail at the edges between images; Gareau et al. (2008) included 10\% overlap in the translation step distance. Spatial rearrangement of the pieces relatively to each other is required in both situations to recover an image of the full tissue section for subsequent volume reconstruction or registration with other modalities. This is usually performed through image registration. Capek et al. (2009) performed stitching by first positioning the tiles using landmark points, and then optimising a similarity measure in the parameter space of translations using $n$-step search (Tekalp, 1995). This method was implemented in GlueMRC (Karen et al., 2003). Hsu et al. (2008) mosaicing by matching features detected in adjacent histological tiles. Those were extracted using wavelet-based edge correlation and pairs of corresponding features were then identified by maximisation of the normalised correlation coefficient. Saalfeld et al. (2012) addressed mosaicing of ssTEM images using previous work (Saalfeld et al., 2010) and SIFT features. 
Table 2: List of open-source registration toolkits/softwares used for histology reconstruction ( $\mathrm{L}$ and NL stand for linear and non-linear registrations respectively).

\begin{tabular}{rlcll}
\hline softwares/packages/plugins & institution & type of transformation(s) & references & used in \\
\hline \hline ANIMAL & McGill (CAN) & $\mathrm{NL}$ & (Collins et al., 1994, 1995) & (Chakravarty et al., 2006) \\
TurboReg (ImageJ) & EPFL (CHE) & $\mathrm{L}$ & (Thevenaz et al., 1998) & (Riddle et al., 2011) \\
UnwarpJ (ImageJ) & EPFL (CHE) & $\mathrm{NL}$ & (Sorzano et al., 2005) & (Wang et al., 2015) \\
MERIT (MeVisLab) & Fraunhofer MEVIS (DEU) & $\mathrm{L} / \mathrm{NL}$ & (Boehler et al., 2011) & (Schwier et al., 2013) \\
bUnwarpJ (ImageJ) & UAM (ESP) & $\mathrm{NL}$ & (Arganda-Carreras et al., 2008) & (Kindle et al., 2011) \\
Elastix & UMC Utrecht (NDL) & $\mathrm{NL}$ & (Klein et al., 2010) & (Alic et al., 2011; Stille et al., 2013) \\
NiftyReg & UCL (UK) & $\mathrm{L} / \mathrm{NL}$ & (Modat et al., 2010, 2014) & (Pichat et al., 2015) \\
VTK CISG & KCL (UK) & $\mathrm{L} / \mathrm{NL}$ & (Hartkens et al., 2002) & (Benetazzo et al., 2011) \\
AIR & USC (USA) & L/NL & (Woods et al., 1998a,b) & (Brey et al., 2002; Beare et al., 2008) \\
ITK & NLM (USA) & L/NL & (Yoo et al., 2002) & (Mosaliganti et al., 2006; Gijtenbeek et al., 2006) \\
ANTs & UPenn (USA) & $\mathrm{NL}$ & (Avants et al., 2009) & (Adler et al., 2014) \\
DRAMMS & UPenn (USA) & $\mathrm{NL}$ & (Ou and Davatzikos, 2009) & (Ou and Davatzikos, 2009)
\end{tabular}

There exist several softwares that automatically perform the task (Piccinini et al., 2013a): in ImageJ, the Stitching ${ }^{1}$ plug-in (Preibisch et al., 2009); Autostitch ${ }^{2}$ (Brown and Lowe, 2007); Mosaic ${ }^{3}$ (Thévenaz and Unser, 2007); XuvTools ${ }^{4}$ (Emmenlauer et al., 2009); HistoStitcher and AutoStitcher ${ }^{5}$ (Chappelow et al., 2011b; Penzias et al., 2016). Only a few studies, such as Ma et al. (2007) using Autostitch, have been accounting for vignetting (Piccinini et al., 2013a). Piccinini et al. (2013a) developed MicroMos ${ }^{6}$ and ensured their tiles had all been flat-field corrected prior to stitching them back together.

An automatic mosaic acquisition and processing system for multiphoton microscopy was described in Chow et al. (2006), along with the importance of normalisation to avoid shading artefacts at the border of tiles. Methods that extend the tiles beyond their boundaries by propagating available structures were also developed in Jia and Tang (2008) and Yigitsoy and Navab (2013). Stitching is extensively studied in the general computer vision literature (Brown and Lowe, 2007).

\section{3D histology reconstruction}

Histology reconstruction methods aim to restore the loss of continuity due to volume slicing. They are based on the assumption that the shape of a biological specimen changes smoothly across sections, but suffers from the various artefacts that affect every section independently during preparation.

When using histology alone, reconstruction algorithms provide representations of structures and their environment in 3D—which helps with subsequent segmentation and classification tasks (McCann et al., 2015)—but one needs to bear in mind that the original shape is unattainable without prior or external knowledge.

Reconstruction algorithms from serial histological slices rely on image registration and consist of optimising the spatial alignment of variously oriented 2D slices relative to each other, while being robust to artefacts following histological preparation. The most straightforward way is to register every slice with its direct neighbour and repeat the process with the following pairs, but this is not robust to errors. First efforts towards the reconstruction and visualisation of volumes from 2D sections relied on this technique and were initiated in the early 1970s (Levinthal and Ware, 1972; Lopresti et al., 1973). A list of company/academic softwares and plugins for histology reconstruction from serial sections is available in Table 1.

Registration is the process of bringing two images (one usually referred to as "reference, fixed or target" and the other as "floating, moving or source") into spatial alignment and deforming the floating image such that it looks like

\footnotetext{
${ }_{1}$ imagej.net/Image_Stitching

2 autostitch.net/

${ }^{3}$ bigwww.epfl.ch/thevenaz/mosaicj/

4 xuvtools.org/doku.php

5 engineering. case. edu/centers/ccipd/content/software

${ }^{6}$ sourceforge.net/projects/micromos/
} 
the reference image (for transformations others than rigid-body). The objective is to estimate the transformation that optimises an energy function. It is usually made of two terms, one referred to as the matching criterion (a distance measure, in a broad sense) and a regulariser, either implicit (by restricting the type of transformation) or explicit (e.g., deformation field filtering, penalty terms, etc.), which controls the transformation and prevents excessive or unrealistic deformations. This definition holds for the rest of the paper. Further details can be found in reviews about (medical) image registration (Maintz and Viergever, 1998; Hill et al., 2001; Zitova and Flusser, 2003; Sotiras et al., 2013) and a report was recently presented in Viergever et al. (2016) to assess whether the goals of the field were met. A list of open-source toolkits for medical image registration is available in Table 2.

Histology reconstruction is obtained by the composition of transformations between all pairs of adjacent sections, starting from a certain reference slice. The quality of the resulting volume depends on both the success of all pairwise registrations and the choice of that reference; it is usually an arbitrary choice made by an expert, who selects a slice that exhibits little deformations, few artefacts and high contrast. Although the first slice (Lee et al., 2005; Colchester et al., 2000) is sometimes chosen as reference (Chen et al., 2003; Krinidis et al., 2003a), it may be preferable to select it around the centre of the stack (Ourselin et al., 2001b; Pitiot et al., 2006; Cifor et al., 2011). This minimises the propagation of errors due to slight misalignments (let aside registration failures), which may produce skewed or helicoidal stacks. To the best of our knowledge, only Bagci and Bai (2010) proposed to automate the process of selecting the best reference slice by considering the information content in feature space.

Without any information about the original shape, volume reconstruction remains an ill-posed problem i.e., although there exists a solution, it is not unique (and the true one is unknown); for example, changing the initial arrangement of slices relative to one another will lead to a different reconstruction. Whichever way it is addressed, the process tends to straighten up structures: a banana-like original volume, cut and reconstructed, will end up looking like an ellipsoid-hence its name, the "banana effect" or "z-shift" effect (Malandain et al., 2004).

Some works tried to bypass registration failures through graph theoretic approaches (Yushkevich et al., 2006; Adler et al., 2014; Pichat et al., 2015), which formulate reconstruction as a shortest path problem in order to identify the best sequence of transformations. Alternatively, most recent works proceed by aligning every slice with a set of neighbouring slices (as opposed to considering only one neighbour) in order to smooth out potential errors and improve continuity (Mertzanidou et al., 2016; Rusu et al., 2015; Saalfeld et al., 2012; Feuerstein et al., 2011; Nikou et al., 2003).

We classify works aiming to reconstruct volumes based on the type of registration they used. This yields two categories: registration using geometric features (\$4.1) and registration using voxel comparison (\$4.2). While the former may be fast (because it uses a subset rather than the whole image domain), the latter is more accurate but slower and requires careful initialisation as methods tend to settle in local optima.

\subsection{Geometric methods (landmark-based)}

Geometric methods aim to register two images by minimising a criterion that takes into account landmark information. The first step in geometric registration is to obtain points of interest (\$4.1.1). Those are usually noticeable locations in the image, under the assumption that saliency at the image level is equivalent to relevant anatomical regions. After finding correspondences between landmarks, a smooth transformation is sought so that their alignment is respected (\$4.1.2). Further details can be found in Sotiras et al. (2013).

Note that although geometric methods can theoretically be used for more complex transformations than rigid and affine (if the sets of points are large enough and well-distributed), intensity-based methods ( 4 4.2) would likely perform better at such task for they use the entire image domain (contrary to features, which would likely be concentrated along contrasted boundaries).

\subsubsection{Detecting points of interest}

Processing histological images is complex when it comes to using points of interest: the appearance of slices vary greatly and even adjacent sections expose similar rather than the same constituents. Consequently, their description should be flexible enough to grant matching, while peculiar enough to disambiguate between close potential candidates. Besides, the very task of locating reliable landmarks remains an open problem, and it is still an active area of research (Sotiras et al., 2013). In this section, we use interchangeably the terms landmark, keypoint and point of interest. Points of interest fall into three categories: manually extracted landmarks, needle tracks, and automatically extracted landmarks. 
Manual landmark selection. It is usually carried out by experienced histopathologists and benefits from the rich details that high resolution histological images provide. The main advantage of manual selection is that it allows for accurate, relatively consistent selection of anatomically relevant landmarks. The task is however very time-consuming and subject to inter- and intra-user variability, and was for example performed in Gaffling et al. (2011). Zhao et al. (1993) manually segmented the contours of the autoradiographs; boundaries may be sampled for point matching, or used as such for curve matching.

Needle tracks as landmarks. Needles can either be inserted in the fresh tissue, or in the embedding medium by placing ink marks (Simonetti et al., 2006). The marks can then be manually or automatically isolated, such as in Colchester et al. (2000) who identified centres of labelled needles tracks using Hough transform. Although the technique is known to be invasive, recent advances allow to minimise damages to the tissue (Hughes et al., 2013).

Automatic feature extraction. Within the context of histology, we identified three main types of features associated with automated methods to extract and describe them, namely Fourier-based, blob-like and object-level features.

Fourier-based features Such features relate to edges extracted via harmonic analysis. Hsu (2011) adapted a method introduced earlier in the context of mosaicing (Hsu et al., 2008) to histology reconstruction, also based on Hsieh et al. (1997). The detection of edges was handled by wavelet transforms. The robustness to noise was achieved using edge correlation, as introduced by Xu et al. (1994). Reliability of feature points was increased by means of multiscale edge confirmation, which filtered out the noise since mostly features remain across multiple scales (unlike noise). The reader may also refer to Mallat's works for a thorough study of multiscale edge detection through wavelet theory (Mallat and Zhong, 1992). The orientation of the feature point was determined through a line-fitting method rather than estimated using the result of the wavelet transform (which is sensitive to noise): it essentially considered a neighbourhood of a detected feature and estimated the orientation of the edge line passing through it and neighbouring edge points. Braumann et al. (2005) used Fourier-Mellin invariant (FMI) descriptors of images (Casasent and Psaltis, 1976). They were obtained by Fourier-Mellin transform of the image in a polar coordinate system, which decoupled translation, rotation and scaling (respectively for rotation and scale invariance). Note that Ghorbel (1994) later showed that using instead the analytical Fourier-Mellin transform allows getting a complete set of similarity-invariant features.

Blob-like features The most popular blob detector in the computer vision literature is surely the Scale-invariant feature transform (SIFT). It is based on local extrema (or blob) detection (Lowe, 1999). The detector relies on difference of Gaussians (DoG), which is an approximation of the scale-normalised Laplacian of Gaussian (related to each other through the heat equation) and thus contains no directional information. Keypoints are local optima in the DoG scale space of the image. Candidate keypoints that are unstable i.e., low contrasted extrema or those lying on edges (since they are invariant to translations along their direction) are discarded. Location, scale and orientation (estimated as the main gradient orientation over a keypoint neighbourhood) are encoded in the descriptor of every keypoint. The interested reader may refer to Toews and Wells (2009) for an efficient encoding of that vector. An in-depth analysis of the SIFT method is available in Rey Otero and Delbracio (2014).

SIFT was used in Koshevoy et al. (2006), and Saalfeld et al. (2012) based on their previous work for the registration of tiled serial TEM sections $^{7}$ (Saalfeld et al., 2010). Wang and Chen (2013) used colour deconvolution (see $\$ 3$ ) to separate hematoxylin and eosin stain contributions from individual histopathological images. Eosinophilic structures were used as object-level features for image registration, from which points of interest were detected using DoG detector. This was reused in Wang et al. (2014, 2015).

Lobachev et al. (2017) used another popular feature detector and descriptor, SURF (Bay et al., 2006). It is based on the determinant of the Hessian matrix operator and relies on integral images for fast computation. As far as SIFT is concerned, DoG is basically a Laplacian-based detector and the Laplacian operator is defined as the trace of the Hessian matrix. Using its determinant (instead of the trace) as it is the case with SURF, discourages the detection of elongated, ill-localised structures.

\footnotetext{
${ }^{7}$ Saalfeld and Tomancák (2008) developed plug-ins for ImageJ to extract SIFT and Multi-Scale Oriented Patches, MOPS Brown et al. (2005) correspondences in two images: http://imagej.net/Feature_Extraction
} 
Ulrich et al. (2014) used Binary Robust Invariant Scalable Keypoints, BRISK (Leutenegger et al., 2011), based on the AGAST corner detector (Mair et al., 2010). Note that an evaluation of binary feature descriptors performance can be found in Heinly et al. (2012).

Nagara et al. (2017) used Accelerated KAZE feature points (Alcantarilla et al., 2012, 2013).

Object-level (or high-level) features We identified two subtypes of such features: (i) anatomical structures and (ii) tissue boundaries (curves and points). The same organisation is followed in the corresponding paragraph of §4.1.2.

(i) One school of thought recommends the use of structures such as vessels, nuclei etc., (Ruiz et al., 2009). The rationale is that traditional feature detection schemes generate a great amount that are regular in appearance, thereby making matching unrealistic. Such features are also described in Gurcan et al. (2009). For example, Schwier et al. (2013) extracted vessel-like structures in greyscale images using thresholding and mathematical morphology in every slice. The sets of structures were then refined using eccentricity, ellipticity and size criteria. Prescott et al. (2006) extracted specific regions in cochlear images using Otsu's thresholding, mathematical morphology and only kept the largest connected components. For each stain type, colour segmentation followed by mathematical morphology allowed Cooper et al. (2009) to segment significant structures such as blood vessels, other ductal structures or small voids within the tissue area.

Other methods, although relying on that same subtype of features (Xu et al., 2015; Ruiz et al., 2009; ArgandaCarreras et al., 2010), address the matching step by comparing informative patches (also referred to as windows, blocks, boxes or tiles) centred around those keypoints. In other words, features are described by the intensities of pixels around them, which comes down to a block-matching strategy to infer correspondences. We thus detail the matching step for such approaches (referred to as tile-based methods) in $\S 4.2$. For the sake of completeness, Xu et al. (2015) extracted relevant structures from the images based on colour and size. Arganda-Carreras et al. (2010) extracted structures of interest by combining fast marching algorithm and level-sets. Ruiz et al. (2009) selected tiles that have rich content i.e., which variance is above a certain threshold.

One successful application of those high-level features has been the registration of differently stained histological sections, although the literature on that problem is relatively sparse (Braumann et al., 2006; Song et al., 2013, 2014). It is a multimodal problem in that every section varies in appearance: images exhibit different colour distributions and different structures due to different staining. This is solved by identifying common structures and grouping them into comparable clusters. The problem thus becomes monomodal using labeled images or probability maps. Braumann et al. (2006) assigned every pixel a "segmentation vector" containing, for successive Gaussian smoothed versions of the image, its RGB value and the colour mean of a neighbourhood around the pixel. The clustering of the image into different numbered classes was based on Pernkopf and Bouchaffra (2005), who selected the number of components that best modeled the distributions in order to represent the characteristics of the images adequately. A similar idea was proposed by Song et al. (2014) except that the segmentation vectors for each pixel, called "appearance feature vectors", also included information about texture. The clustering of the appearance feature vectors was carried out using a principal eigenvector binary tree clustering algorithm.

(ii) One last type of approaches consists of using solely the tissue boundary (probably the highest-level feature), whereby the images simplify to a single curve. After extraction, those are used for contour matching. Extracted curves may also be sampled to perform point matching (those points are also referred to as nodes). Tissue edge points sampled along boundaries have the advantage of being less vulnerable to e.g., tearing-when sampled appropriately-from which intensity-based methods would suffer. However, their detection relies on accurate segmentations, which in turn may be affected by intensity inhomogeneities if, for example, a simple global threshold is to be used for all the slices. Contours of tissues were obtained automatically by thresholding in Shojaii et al. (2014); Shojaii and Martel (2009); Cohen et al. (1998). They were manually edited (if necesssary) in Cohen et al. (1998) and modeled using B-splines; the inverse chord length method (Cohen Fernand et al., 1995), which regulates the number of knot points based on the amount of shape variation a region is subjected to, was used to select the set that best described a given curve (fewer knots when the variation is small). Rangarajan et al. (1997) extracted the locations of high confidence edges (including tissue boundaries) by thresholding Canny edge images (Canny, 1986). Tan et al. (2007) extracted the sharpest curvatures along the contours of tissues, which yielded three feature points at consistent locations in every section. The tractability across slices allowed for the computation of "trajectories". Krinidis et al. (2003b) obtained contours using a 2D elastic physics-based deformable model. The model consisted of a set of nodes, initially 
distributed over a circle. In its fully deformable configuration, the model allowed each node to move independently, without affecting adjacent ones. Guest and Baldock (1995) extracted two types of nodes with the aim of creating a mesh: the object nodes were automatically selected, ideally along the boundary of the structure i.e., with large gradient, and a minimum distance criterion to prevent them from being too close. The background nodes were sought in the background region with larger minimum distance. Delaunay triangulation from the obtained nodes provided a mesh with higher density over the domain of the tissue.

\subsubsection{Correspondences and spatial transformations}

Correspondences between landmarks may be straightforward, as it is the case when extracted manually (although labour intensive and time-consuming) or using segmented needle track holes. For example, Gaffling et al. (2011) computed the trajectories of landmarks (placed at identifiable locations along the tissue outer and inner boundaries) tracked across the slices through a fourth order polynomial fit. Note that polynomial transformations are usually advised to be computed using a low-degree polynomial due to noise and numerical instabilities (Ali and Cohen, 1998).

Using needle tracks. Holes from the tracks allow for the computation of a (linear) transformation by least-squares minimisation. Colchester et al. (2000) used a set of photographs of both anterior and posterior faces of every tissue sections. "Within-slice co-registration" was achieved by minimising the sum of squared distances between centres of labelled needles tracks for every pair of faces. Then, "between-slice co-registration" consisted of registering the posterior face of one section with the anterior face of the next using block-matching (Ourselin et al., 2001b). Other examples of use of fiducial markers include works from Goldszal et al. (1995) and Humm et al. (1995). Although they may increase the reliability of the registration process because their locations are easy to track in the images, needles also damage the tissues and introduce bias if the cutting plane is not orthogonal to the needles axes. This protocol was however improved in Gibson et al. (2012) and Hughes et al. (2013).

Using automatically extracted features. In contrast, automatically extracted features require a dedicated step that seeks for correct matching pairs in order to derive the correct transformation. Automatically discarding false matches is critical; otherwise the latter methods would suffer from the same problems that hamper intensity correlation (Rangarajan et al., 1997).

Using Fourier-based features Hsu (2011) used an analytic robust point matching method for global registration. The alignment was refined using a feature-based modified Levenberg-Marquardt algorithm (Moré, 1978). Braumann et al. (2005) matched FMI descriptors between a reference and a target image using a symmetric phase-only matched filtering (Chen et al., 1994). The parameters of rigid transformations were derived from it.

Using blob-like features Matching pairs are usually found by minimising the Frobenius norm in the descriptor space. Random sample consensus, RANSAC (Fischler and Bolles, 1981) is then used to discard wrong correspondences and to solve for the transformation. Koshevoy et al. (2006) assumed that serial section transmission electron microscopy (ssTEM) images were taken at the same scale, and suffered from minor deformation on the global scale, which made the scale-invariance requirement unnecessary. Only SIFT descriptors belonging to the same octave and scale of the DoG scale space thus needed to be compared against each other. To this end, Koshevoy et al. used an optimised $k \mathrm{~d}$-tree with a best-bin-first nearest neighbour search algorithm (Beis and Lowe, 1997). Wrong correspondences were filtered out using a criterion based on Euclidean distances, similar to that introduced in Hsieh et al. (1997). Lobachev et al. (2017) matched SURF descriptors using a bi-directional brute-force matcher.

Solving for the transformation parameters was achieved using RANSAC in Koshevoy et al. (2006); Wang and Chen (2013); Wang et al. (2014, 2015); Lobachev et al. (2017); Ulrich et al. (2014); Nagara et al. (2017). It essentially estimates the set of feature points that behave consistently with respect to a linear transformation. Saalfeld et al. (2010) estimated simultaneously the rigid arrangement of tiles within and across sections using SIFT features and RANSAC registration. The methods are available online ${ }^{8}$. It was extended in Saalfeld et al. (2012) by refining the alignment

\footnotetext{
${ }^{8}$ Two stand-alone plugins were implemented: Elastic Montage, for mosaicing, and Elastic Stack Alignment, for the alignment of images from serially sectioned volumes. They are incorporated in the TrakEM2 software and available at http://imagej.net/Elastic_Alignment_and_ Montage
} 
using intensity-based registration (a block-matching strategy detailed in $§ 4.2$ ). The combination of both strategies was used to initialise an elastic registration, for which each image was tessellated into a mesh of regular triangles. Like in Guest and Baldock (1995), the system of equations representing the whole stack of slices was an elastic spring finite element model. The entire system stabilised when the sum of the forces of all springs was close to zero.

Lobachev et al. (2017) computed an additional non-rigid transformation at multiple resolutions using feature pairs in adjacent sections, in order to constrain the control points of B-splines. At every iteration, the feature size decreased while the resolution of the grid of control points increased. No reference section was taken and all the images were deformed towards a minimum energy function. Ulrich et al. (2014) aligned all BRISK feature pairs by least-squares deformations. This process was repeated if the pairs after transformation were not stable. The methodology was reused in Lobachev et al. (2017) and incorporated into a multi-resolution framework.

Using high-level features (i) The Euclidean distance in combination with other criteria, like the size of structures, is used to assess the similarity between pairs of such features. Schwier et al. (2013) tried all possible combinations of pairs in adjacent sections. The transformation that gave the best similarity was kept. The matching cost took into account a distance range, within which matching pairs should lie, as well as the closeness in terms of object size (area). This was robust to cases where no valid correspondence was found. Pairwise non-rigid registrations (Modersitzki, 2004) were performed and implemented as part of a software (Boehler et al., 2011). Prescott et al. (2006) paired features using the area only. Mismatches were identified using a distance-based criterion, similar to that presented in Koshevoy et al. (2006). The linear transformation was derived from a matching graph, in which every node is a matching pair and is associated with a transformation. An edge exists between two nodes if the two transformations are sufficiently similar. The global transformation is the average of those constituting the maximal cyclic structure from the graph. It served as an initialisation for subsequent registration based on maximisation of mutual information and gradient information (Pluim et al., 2000). Cooper et al. (2009) paired features based on both size (area) and eccentricity. Mismatches were filtered out in a way similar to Prescott et al. (2006) and rigid transformation parameters were associated with every correct pair. The correct rigid transformation was then estimated through a voting scheme. Non-rigid registration was performed using second-order polynomials, which coefficients were calculated using correct feature pairs.

When registering differently stained histological sections, labelled classes must be matched to corresponding common structures in the images. Braumann et al. (2006) applied a methodology partly based on Braumann et al. (2005) and reused in Wentzensen et al. (2007): after clustering the images into different numbered classes, class label adjustment was performed in cases where the assignment was not consistent. The problem of having more and/or different classes - because sections may exhibit different structures-was tackled by merging classes so as to have the same regions segmented in both images. Every labelled image pair was finally aligned by computing the displacement vector field using non-linear, non-parametric curvature-based registration. Song et al. (2014) solved the correspondence problem by grouping clusters into three superclusters (called "content classes") in each image according to various partition schemes. The pair of partitions that maximised mutual information provided the optimal content classes in each image. These classes were then refined using spatial features. Then, each image was transformed into a multichanel probability map, where each channel corresponded to one content class. Block-matching registration was performed between pairs of probability maps for each channel independently. This provided a displacement field, from which a non-rigid transformation was estimated using a regularised least squares difference minimising method.

(ii) Cohen et al. (1998) matched tissue boundaries from adjacent sections, modeled as B-splines, by comparing their knot points. The major drawback associated with B-spline representations is the non-uniqueness of the set of control points, which hampers the comparison of curves. This was solved in Ali and Cohen (1998) (see §5.1.2) using the intrinsic features of curves, which properties derived from the Frenet frames (Millman and Parker, 1977). Zhao et al. (1993) affinely registered slices of autoradiographs by minimising displacement of manually segmented contours (using sum of squared differences, SSD) by analysis of point-to-point disparities in two images: a boundary point in one section differs from its corresponding point in the adjacent section by a disparity vector. Trahearn et al. (2014) used Curvature Scale Space (Mokhtarian and Mackworth, 1986) to represent shape (the tissue boundary) at various scales and register whole-slide images of histological sections.

When sets of points sampled along the extracted boundaries of tissues are to be matched, one popular method is the Iterative Closest Point (ICP) method (Besl and McKay, 1992). Shojaii and Martel (2009) used ICP to register every histological slice with its corresponding blockface photograph. Points were uniformly distributed along every 
smoothed boundary of the tissue by excluding high curvatures using a rolling-ball filter. Shojaii et al. argued that "high-curvature boundaries might lead ICP to converge to local minima and deteriorate its robustness". Deformable registration was then performed using thin-plate spline (TPS). Rangarajan et al. (1997) optimised simultaneously the affine transformation parameters and the one-to-one correspondences between two sets of edge points in adjacent sections. This method was referred to as robust point matching. Krinidis et al. (2003b) found correspondences between contour nodes of adjacent slices using an affinity matrix. Corresponding nodes between adjacent slices were couples which relative distance was lower than a certain threshold. False matching were filtered out by global affinities, which ensured that correct correspondences also exist in slices further away. Translation and rotation parameters were computed by minimising the mean square error between pairs of matching nodes. Gaffling et al. (2011) used the offsets of every landmark to a smooth curve representing the trajectory of that landmark across slices to compute a sparse displacement field for every slice; the vector fields were then densified (Fischer and Modersitzki, 2003). Every image was finally deformed such that its landmarks lied on the trajectories, and every following slice was then registered to it. A similar strategy was followed earlier by Tan et al. (2007), where three edge points in every slice were used as control points of three non-uniform rational B-spline curves (trajectories).

\subsection{Iconic methods (intensity-based)}

Histology reconstruction can also be achieved by means of intensity-based registration. The main difference with the geometric methods of $\S 4.1$, is that - as their name suggests-iconic methods are based on voxel intensities instead of features. This means the distance-optimisation framework (where the distance can be a similarity measure) is applied to the entire image domain. In that sense, they can potentially be better at estimating a dense deformation field (feature-based methods require interpolation, which makes them less accurate when the set of landmarks is sparse). However, their efficacy comes at a computational cost.

In the following, we present reconstruction methods that relied solely on linear registration, and others that were complemented with non-linear registration so as to improve the continuity of anatomical structures in 3D.

Linear transformations. Andreasen et al. (1992) optimised the parameters of every rigid transformation by minimising a weighted SSD between the intensities of two adjacent slices. Weights were defined as the ratios of intensities of both images. More recently, methods more robust to intensity variations across slices have been proposed. Blockmatching (Ourselin et al., 2000) was used in Dubois et al. (2007) and Ourselin et al. (2001b); it consisted of (i) finding corresponding blocks from two images by maximisation of a certain similarity criterion within an exploration neighbourhood and (ii), using the resulting displacement field, filtering out mismatches via least trimmed squares (LTS) (Rousseeuw, 1984) in order to compute a robust, global rigid transformation between the two images. Nikou et al. (2003) defined a local energy function that was optimised sequentially in order to bring into rigid alignment every unvisited slice with a group of neighbouring slices. They used M-estimators as cost functions, which aimed to reduce the effect of outliers in the regression process by replacing the square function of the residual in the standard least square minimisation by the German-McClure $\rho$-function. Dubois et al. (2007) jointly reconstructed histological and autoradiographic volumes by first stacking sections using their centres of mass, and registering pairs of adjacent sections for each stack using block-matching. The histological volume was then used as a reference for the refinement of the reconstructed autoradiographic volume (2D-2D registration between autoradiographs and histology), due to the fact that inner anatomical structures of the brain are more visible in histology.

Non-linear transformations. Other authors employed non-linear transformations (Gefen et al., 2003) to reach smoother reconstructions. Such methods must be initialised with a linear registration (or with manual alignment, or by aligning image centres). One should note that initialisation is a non-trivial and non-negligible step, and this statement holds for $\$ 5$ too. The additional use of non-linear registration is often a source of discussions in the literature (Lee et al., 2005; Dubois et al., 2007). On the one hand, these transformations can provide a better overlay and grant richer quantitative analyses. On the other hand, since no external information about the shape of the tissue is available, bias may be introduced by choosing one slice as the reference shape.

Cifor et al. (2011) guaranteed the smoothness of the reconstructed volume in three steps: the volume was initially reconstructed using pairwise rigid registrations. Next, boundaries of interesting structures were extracted (by thresholding) in every 2D section and smoothed using a min-max curvature flow constrained to 2D (out-of-plane)using a mean curvature flow in Cifor et al. (2009). This provided a sparse displacement field computed over pixels 
along the initial boundary of the extracted surface, then extrapolated to the entire set of slices. Smoothness was also used as a criterion for histology reconstruction in Casero et al. (2017), where the refined alignment of the stack was a solution of the heat diffusion equation. The algorithm alternated between the updates of slices' transformations and their neighbours' transformations until convergence. Finally, accumulated transformations updates were applied to each slice. The same purpose was followed in Gaffling et al. (2015), where the reconstruction of a stack of histological slices was formulated as an iterative Gauss-Seidel update scheme applied to images, using by definition two adjacent slices (above and below) and modified to also include information from the image itself (Gaffling et al., 2009) - thereby, they also showed that a small neighbourhood is sufficient to restore smoothness. That scheme allowed for smoothing high-frequency perturbations more than lower frequencies associated with the progression of anatomical structures along the stack (as it is assumed to vary slowly enough by nature of the histology process). A similar strategy was followed earlier in Krinidis et al. (2003a), using iterated conditional modes (Besag, 1986) for the optimisation of a global energy function that quantified similarity between slices.

Ju et al. (2006) represented deformations by independent single valued functions in horizontal and vertical directions: they considered that every 2D warp can be decomposed into 1D piecewise linear deformations with elastic constraint in $x$ and $y$. The minimisation of the error function for registration was achieved by means of an extension of the dynamic time warping in 1D (Sakoe and Chiba, 1978) to 2D problems.

Wirtz et al. (2004) first rigidly registered slices using principal axis transformations, and then performed multi-scale non-linear registration with a regularisation based on elastic potentials. The system of Navier-Lamé equations was linearised by means of a non-linear Gauss-Seidel iteration method and approximated by finite differences. This was extended in Wirtz et al. (2005) by replacing the SSD similarity measure in the variational formulation with a weighted combination of two derivative-based (respectively gradient and Laplacian of the image) SSD measures. The error function was thereby less sensitive to intensity inhomogeneities.

Braumann et al. (2005) performed non-rigid registration on rigidly pre-aligned slices in two steps. First, they used polynomial warping on luminance-transformed images: correspondences between control points of adjacent slices were used to estimate the polynomial coefficients through the minimisation of a least-square error. This provided a sparse displacement field. Then, a curvature-based registration (Fischer and Modersitzki, 2003) was performed on staining-based tumour probability maps. Such maps reduced artefacts around the tumour and thus eased the registration. Braumann et al. also suggested to skip the intermediate polynomial registration as improved performance is expected using a multi-grid scheme for the curvature-based registration.

Pitiot et al. (2006) proposed a method that computed a global non-linear transformation by elastically interpolating between linear transforms defined on pairs of sub-images (hence the name of "piecewise affine registration"). These sub-images represented geometrically, and often anatomically, coherent components. They were automatically extracted through clustering of an initial displacement field (Ourselin et al., 2000) computed between the images to be registered.

Feuerstein et al. (2011) formulated the problem of optimising transformation parameters for every slice relative to, simultaneously, a reference image and the two neighbouring sections, as Markov random fields. The Markov random fields energy is composed of unary potentials, which account for the registration to the reference images and pairwise potentials, which encode the registration to neighbouring slices and the regularisation of the displacement field. This formulation served as basis for the deformation field model in Müller et al. (2014).

Brandt et al. (2005) an initial affine registration followed by non-rigid registration, were performed both by maximisation of the NMI. The latter transformation was modeled as a cubic B-spline free-form deformation (Sederberg and Parry, 1986).

Schmitt et al. (2007) initialised the reconstruction by registering slices affinely using a variant of principal axes transformation-PAT (Alpert et al., 1990). They adopted the stochastic interpretation of PAT presented in Modersitzki (2004) (p.45), in which the images are represented as Gaussian density functions. The problem was formulated as the estimation of a density that best fits a set of reference densities in the sense that the Kullback-Leibler distance is minimised. Due to the lack of robustness of Gaussian distributions to perturbations, such as tears, wrinkles, torn out pieces, artefacts etc., Schmitt et al. used Cauchy density functions instead, on which robust PAT relies. Standard PAT was used in Cooper et al. (2006). This was followed by two partial optimisations of the SSD with respect to the shear first, and then to rotation, shearing and translation. Finally, elastic deformation was performed, similar to that used in Saalfeld et al. (2012).

The only work, to the best of our knowledge, explicitely addressing the problem of histology reconstruction with missing slices was found in Gaffling et al. (2009) and dealt with by interpolating them. Images adjacent to a missing 
slice were non-rigidly registered using the variational approach of the problem defined in Modersitzki (2003). The interpolated deformation field, which is a fraction of the resulting deformation field depending on the gap between the two registered images, is applied to the template image to approximate the missing intermediate slice.

Tile-based approaches. These methods, introduced in $\S 4.1 .1$, use patches of the image as features and are therefore similar to block-matching when establishing correspondences: Ruiz et al. (2009) computed the translation and rotation parameters that related corresponding blocks in two images; those were identified within a sufficiently large area of the reference image by maximisation of normalised cross-correlation. This step provided a sparse displacement field and a second-order polynomial transformation was computed, which coefficients were obtained using least squares. Xu et al. (2015) explicitely based their method on Ourselin et al. (2000). Arganda-Carreras et al. (2010) proposed a shape-based rigid registration method. After an initial rigid-body registration between adjacent sections, correspondences between structures of interest were sought for by means of bounding boxes around them. Overlapping boxes in adjacent sections were assigned the same group label. Two bounding boxes overlapping in the same section were grouped into a supergroup. Remaining ungrouped structures were assigned the number of the closest group/supergroup in the adjacent section. Registration of every grouped structures was performed using the phase correlation method. Correction of remaining misalignments was achieved by elastic registration using B-splines in a multiresolution framework. Auer et al. (2005) kept meaningful tiles (called "subimages") that satisfied a variance criterion. Their centres were used as control points for TPS registration. TPS was preferred for its physical properties but Auer and colleagues outline that it highly depends on the control points, as opposed to e.g., B-spline interpolation.

\section{Histology reconstruction using 3D medical images}

This section presents pipelines that aim to improve histology reconstructions with the help of 3D medical images. As mentioned earlier, this supposes the access to a suitable set of histological slices. By suitable we mean that a sufficient number of sections with an appropriate spacing between them (relative to the MRI slices thickness) is available. Hence the slight abuse of language made in the section (and the paper) title in cases where only a single or too few histological slices are available: the purpose is no longer histology reconstruction but the correspondence problem remains the same and calls for 2D-2D or 2D-3D registration techniques. Since those techniques are not limited to single-section studies - and could well be applied to a group of sections (Nir et al., 2014), we also describe them in the following.

Combining histology and medical imaging dates back to the late 1980s. Early attempts include works by Sze et al. (1986), who aimed to provide histological explanation for high intensities detected routinely in MR, and Nesbit et al. (1991), who studied the pathogenesis of multiple sclerosis using MR, computed tomography (CT) and a biopsy.

The process of combination benefits from the heterogeneity and multiple resolutions of the images. In the end, it serves to increase the specificity of medical imaging analysis with baseline information about the actual properties of the underlying biological tissues (Annese, 2012): since medical imaging provides only indirect information, it is essential to show that resultant findings correlate with pathological findings.

Multimodal works treat their different images as separate entities: the terms (co-)registration, (co-)alignment, matching, mapping or warping are used interchangeably in the covered literature. They all provide additional, combined information in the form of overlays for diagnostics, treatments, quantification etc. The term "fusion" is also commonly used but it should be distinguished from "data fusion" in the sense of creating a single composite image from different sources via numerical fusion operators, extensively reviewed by Bloch (1996) and more recently by James and Dasarathy (2014) for medical images.

The section is structured according to the modalities involved in the registration process: (i) registration of histology with ex and/or in vivo 3D medical imaging (\$5.1): this includes cases where ex vivo is used as an intermediate modality when relating in vivo imaging with post mortem data; (ii) registration of histology with ex and/or in vivo 3D medical imaging using blockface photographs as an intermediate modality (§5.2): this also includes cases where both blockface photographs and ex vivo are used as intermediate modalities.

\subsection{Histology $\leftrightarrow 3 D$ medical imaging}

Multimodal registration between histological slices and 3D medical imaging can be addressed in three ways, summarised in Fig. 3: 


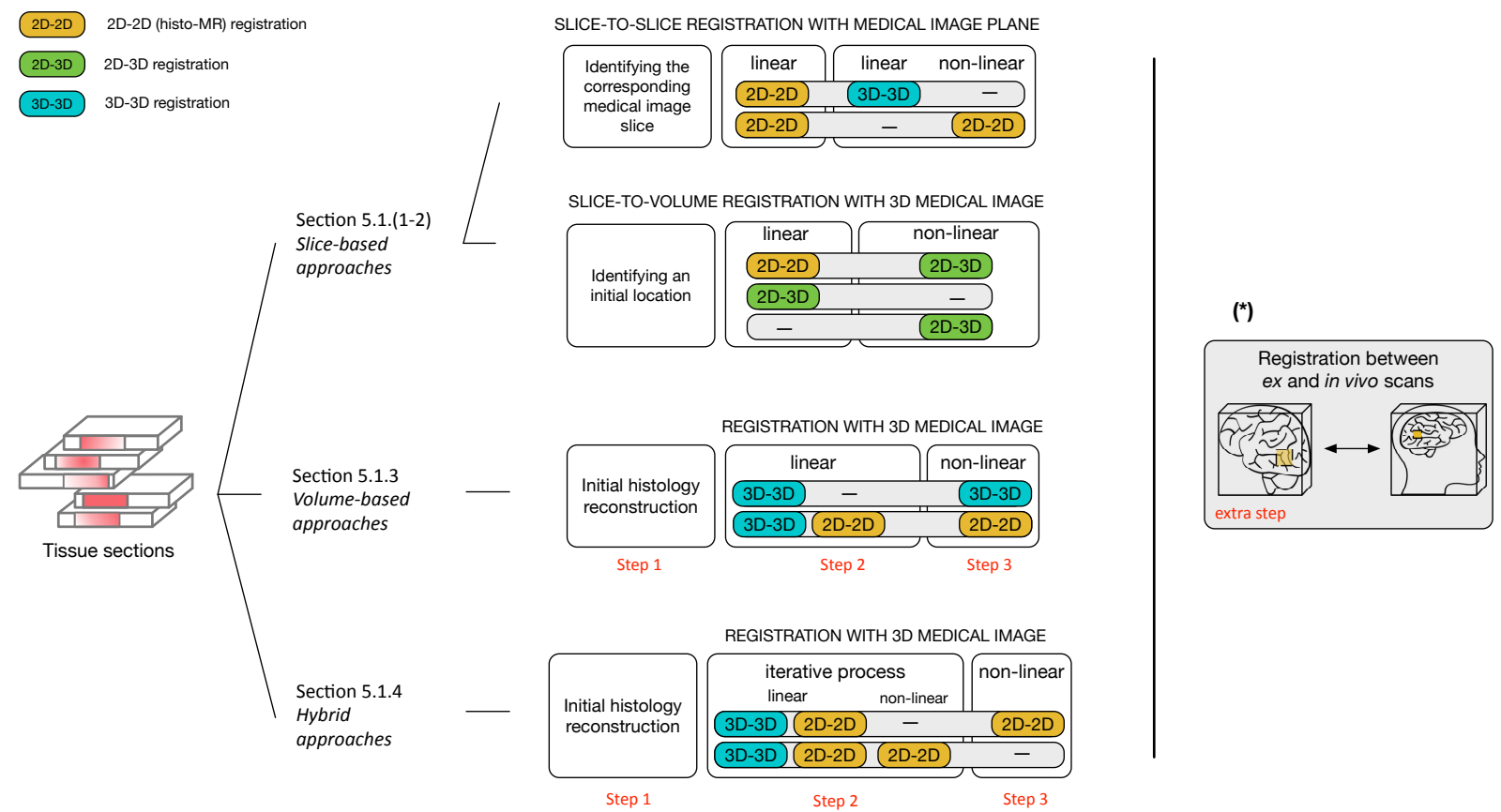

Figure 3: Strategies to register histology with volumetric medical imaging (ex or in vivo alone). The three main approaches (slice-based, volume-based and iterative) are presented. (*) In cases where ex vivo imaging is used as an intermediate modality, correspondences between ex vivo and histology are achieved through steps 2 and 3, and the mapping between histology and in vivo is completed via registration between ex and in vivo scans (extra step).

- Slice-based approaches (\$5.1.1 and 5.1.2) They consider every histological slice as an individual object. Those may be preferred over volume-based approaches in cases where e.g., the histological dataset is too sparse or has too few slices. The alignment between histology and medical imaging is then carried out using either (i) slice-to-volume (2D-3D) registration or (ii) slice-to-slice (2D-2D) registration, which is a simplification of (i); both require careful initialisation i.e., the identification of a "corresponding" plane in the medical image volume.

- Volume-based approaches (\$5.1.3) They consider the set of histological slices as a whole and therefore rely on an initial histological volume. The main goal of initial reconstruction is to correct for the various orientations that the tissues may have across slices (when mounted on glass slides) in order to facilitate subsequent registration with 3D medical imaging. It provides better support and aids the optimisation of the cost function. More complex initialisations have also been developed, which intend to be more robust to registration failures. Both the alignment and the reconstruction are then refined in various ways.

- Hybrid approaches (\$5.1.4) They also rely on initial histology reconstructions and alternate between volume- and slice-based approaches so as to get the best out of the two worlds: a more accurate histology reconstruction for a refined alignment with clinical imaging and vice versa. They repeat until the reconstruction has converged.

\subsubsection{Slice-to-slice approaches $(2 D-2 D)$}

These methods assume that the cutting planes of histological slices and the acquisition planes of the 3D medical image are parallel and that there always exists a histological section that has a counterpart in the set of MR slices. The problem therefore simplifies to a $2 \mathrm{D}-2 \mathrm{D}$ registration between every histological slice and its corresponding slice in the 3D medical image.

Visual selection. Slice correspondences can be achieved visually (Gangolli et al., 2017; Chappelow et al., 2011a; Mazaheri et al., 2010), in which case an expert radiologist is most commonly asked to identify the MRI slice corresponding to a histological slice on the basis of anatomical landmarks. 
Chappelow et al. (2011a) took advantage of all the data to drive image registration using a multivariate formulation of mutual information, while Mazaheri et al. (2010) performed rigid alignment of the images' centres of mass, followed by $2 \mathrm{D}$ affine registration and finally 2D non-rigid registration using free-form deformations (FFD) (Rueckert et al., 1999).

Li et al. (2006) used TPS transformation to register the in vivo MR plane with its visually corresponding histological slice. It was a smooth registration based on specified corresponding landmarks. The optimal number of landmarks was evaluated as a minimiser of the non-rigid registration error.

Gangolli et al. (2017) manually extracted landmarks at visually matching locations along the tissue edges, within and at the boundary between grey and white matter. Then, a forward non-linear moving least squares transformation (Goshtasby, 1988) was applied to register the histological section with the MRI slice.

Nagara et al. (2017) identified the cutting plane of the specimen thanks to a mark made by a clinician on the $\mu \mathrm{CT}$ prior to histological preparation. Both modalities were first linearly aligned using AKAZE feature points in combination with RANSAC; 2D-2D non-linear registration between every pair of slices from histology and $\mu \mathrm{CT}$ was then performed and formulated as discrete Markov random fields.

Automatic selection. Automated selection of corresponding slices can be achieved via the optimisation of a similarity measure between each histological slice and every slice of the 3D medical image. This assumes that the maximum similarity is obtained when actual corresponding slices are compared. However, Xiao et al. (2011) showed that both visual and automated approaches failed to reliably determine slice correspondences mostly due to the alteration of the tissue during the histology preparation i.e., direct comparison of images from different modalities is a non-trivial task which is prone to errors. Rather, they proposed to compare the set of histological slices with all possible subsets of equal number of in vivo MR slices using mutual information. These subsets were ranked based on cumulated similarity. A group of top-ranked MRI subsets was retained and their lists of correspondences were averaged. The final list was used for 2D affine registrations between slices from both modalities followed by 3D affine registration.

In the clinical literature. Slice-to-slice approaches seem to be favoured for the visual control they allow. However, in most of the works the problem is only partly stated and adressed (Harkins et al., 2015; Kilsdonk et al., 2016; Hammelrath et al., 2016): Lopez Gonzalez et al. (2016) performed linear registrations between ex vivo MR slices and histological images, and these were then visually matched to the closest (in vivo) 3T MR slice.

Harteveld et al. (2016) and Van Der Kolk et al. (2015) manually matched histological sections to their corresponding MR planes using the marked locations from fiducials in the MR images, ink markings in the histologic sections, and gross morphologic features.

Nakagawa et al. (2016) visually identified the histological sections that were morphologically close to the $T_{1 \rho}$ mapping image, and only those were stained.

Koh et al. (2016) compared the tissue sections of the largest cut surface of the tumour side-by-side with MR imaging.

State of the art data analyses therefore rely on two rather loose assumptions:

(i) The sampling during histology is consistent and can coincide with that of the MRI. However, it is for example well-known that thermal expansion of the tissue when a new block face is exposed during microtomy causes uneven thickness of histological sections. Otherwise, one counts on interpolation whereby, depending on resolution, new and uncertain content is created.

(ii) The histological cutting planes are parallel to each other and to the MRI acquisition plane. One counter-argument directly follows from (i); another, from the fact that it is very difficult to ensure that the specimen lies prefectly flat at the bottom of the cassette during histology processing (this is especially true when tissue is the frozen).

As a result, apparatus have been developed to help cutting the specimen at the same interval and orientation as the MR images, as proposed by Drew et al. (2010); Trivedi et al. (2012) in the context of prostatectomy, or by means of 3D-printed brain holders (Absinta et al., 2014; Guy et al., 2016) but their use is not so common. The error made when selecting the closest MR slice was considered in Steenbergen et al. (2015), and the consequences of differences in sampling were noted in Martel et al. (2016) in the specific case of vascular trees from the femoral trochlea. By disregarding such approximations, one needs to be aware that wrong correspondences are very likely to be established and this directly affects, for example, statistical analyses. Similarly, these challenges were described by Meyer et al. 
(2013) and numerous erroneous assumptions made during the process of alignment were listed in the case of prostate cancer.

In some cases where the medical image has low resolution (thick slices) and the histological dataset is sparse or limited to a thin slab of a few consecutive slices, or when the contrast/quality of histological slices is poor, one should however acknowledge that visual selection of corresponding slices, manual alignment of images, manual selection of landmarks or manual initialisation remains the safest and quickest way to address multimodal registration.

\subsubsection{Slice-to-volume approaches $(2 D-3 D)$}

Slice-to-volume approaches extend methods presented in $\$ 5.1 .1$ by acknowledging that nothing ensures that the cutting plane of histological slices is parallel to the 3D medical image acquisition plane. Likewise, there is no guarantee that the histological slices are parallel to each other. This means that structures belonging to a tissue slice may extend over several 3D medical image slices, or in other words, a histological slice may lie obliquely in the medical image volume. This in turn suggests that the corresponding 3D medical image slice can only be found through a slice-to-volume (2D-3D) registration. Reviews on slice-to-volume registration can be found in Markelj et al. (2012) and Ferrante and Paragios (2017).

Landmark-based. Khimchenko et al. (2016) rigidly registered a histological section with a $\mu \mathrm{CT}$ volume using a density-driven RANSAC for plane fitting (Chicherova et al., 2014) and relied on SURF keypoints detected in both histological and each $\mu \mathrm{CT}$ slices. The resulting 3D point cloud had an increased density of matches at the correct location of the histology section, and this was used as a criterion to filter out incorrect pairs. The random sampling of RANSAC plane fitting was thereby biased towards those points that were close to the $\mu$ CT plane of interest. The alignment between the interpolated plane and histology was further refined using 2D Demon registration tool ${ }^{9}\left(\mathrm{Kroon}^{\circ}\right.$ and Slump, 2009).

Gibson et al. (2012) utilised well-arranged strand-shaped fiducial markers, which allowed for the determination of the location and orientation of each section. First, a 2D-3D affine transformation that mapped a fiducial histological slice to its corresponding points on the MR image was found by minimisation of the residuals. Then, a 2D-2D affine transformation mapping each histology slice to its counterpart in the MR was computed using spatial information from all fiducial markers. Finally, the fiducial correspondences were refined using a local optimisation and one last affine transformation was computed using the affine transformation from the previous step as initialisation. Using non-anatomical fiducials was argued to provide robustness to variations in the appearance of the prostate on MR and histology images.

Ali and Cohen (1998) approached registration as a contour matching problem and the multimodal registration problem thereby becomes monomodal. The contour curves were described by means of sets of affine invariants constructed from the sequence of area patches bounded by the contour and the line connecting two consecutive inflections. The affine transformation was estimated from matching vertices using the least square error estimation method.

Intensity-based. Nir et al. (2014) aimed to find the poses of all the histological slices such that the transformed segmented histology slices optimally matched the corresponding re-sliced images of the 3D medical image. They made use of particle filtering to model pose uncertainty, where each particle represents a combination of histological slices in various 3D poses, and derived optimal affine registration parameters in a Bayesian approach. The admissible space of 3D poses was constrained such that the transformed slices do not intersect.

Osechinskiy and Kruggel (2010) proposed and compared geometric transformations that combined a rigid alignment with a 3D deformation field parametrised by various classes of spline functions, various similarity measures, different optimisation algorithms and different optimisation strategies.

Kim et al. (2000) used polynomial transformations to warp an initial in vivo MRI slice that produced minimum error (when compared with all the histological slices). The parameters of a low-order polynomial transformation between the MRI slice and the histological section were optimised by minimisation of the SSD and the correlation coefficient. This method was reused in Zarow et al. (2004) and Singh et al. (2008).

\footnotetext{
${ }^{9}$ Code is available on MathWorks File Exchange.
} 
Gefen et al. (2008) proposed a non-linear registration method to align histological brain sections with a volumetric brain atlas. They started with an image to planar surface matching, during which sections were linearly matched with an oblique slice automatically extracted from the atlas. An image-to-curved surface matching was then performed, during which each section was matched with its corresponding image overlaid on a curved-surface within the atlas. Specifically, a PDE-based registration technique was developed that was driven by a local NMI similarity.

\subsubsection{Volume-based approaches $(3 D-3 D)$}

The main drawback of slice-based approaches is their sensitivity to initialisation, as the landscape of cost functions is very complex and this conditions the convergence behaviour. Other challenges involve the cost function selection and the optimisation strategy (Osechinskiy and Kruggel, 2010). In addition, the information from a single histological section is used alone and its content may be, if not corrupted (see $\$ 2$ and 3), at least difficult to manipulate on its own in a multimodal registration problem. As a result, the performance of slice-to-volume registration shows greater dependence on the input images than 3D-3D registration (Osechinskiy and Kruggel, 2010). Considering histological sections all together i.e., the histological dataset as a whole, allows overcoming such an issue. Volume-based approaches follow Procedure 1.

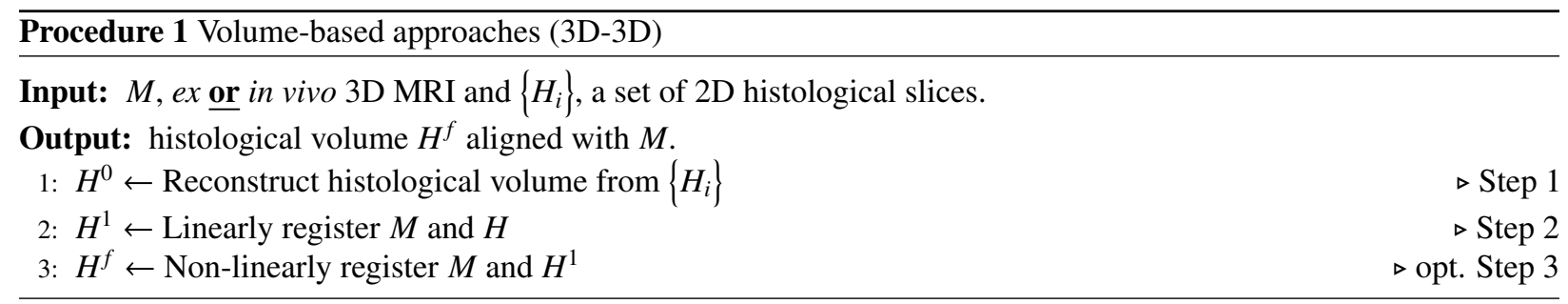

Initial histology reconstruction. (Step 1) can be achieved in several ways and the reader is referred to $\S 4$ for a more complete list of methods dedicated to that purpose. Simple stacking by alignment of centres of mass was used by Goubran et al. (2013). The most common way however consists of serial pairwise linear registrations and provides roughly aligned though satisfactory enough initial volumes (Delzescaux et al., 2003; Malandain et al., 2004; Li et al., 2009; Ou and Davatzikos, 2009; Ceritoglu et al., 2010; Alic et al., 2011; Yang et al., 2012; Stille et al., 2013). In particular, Ceritoglu et al. (2010) registered every slice with its successor starting from the bottom of the stack and repeated the process starting from the top of the resultant stack. In contrast, Stille et al. (2013) started the process from the middle of the stack (the most central slice with minimum artefacts was manually picked) and registrations between pairs of adjacent slices after aligning the centres of mass of masked images were performed. Maximisation of mutual information was used in Ou and Davatzikos (2009), and block-matching in Malandain et al. (2004) and Yang et al. (2012). Initial reconstruction may also be achieved by use of fiduciary rods, such as in Humm et al. (2003), for which the Euclidean distance between corresponding segmented holes from pairs of adjacent slices was minimised.

Other reconstruction methods consider neighbourhoods around slices (as opposed to a single adjacent slice) in order to improve the consistency of the resulting volume. Chakravarty et al. (2006), every slice was registered with both its successor and its predecessor and applied the average transformation to the original slice so that the transformed slices match both their neighbours simultaneously. A similar strategy was used by Rusu et al. (2015). Yushkevich et al. (2006), the size of the neighbouring was extended up to five slices away. A weighted graph was built, with slices as vertices, edges symbolised registrations, and weights were given by an information- and distance-based measure. The shortest path from every vertex in the graph to a specific reference slice was found using Dijkstra's algorithm, and incidentally favoured slices that registered well (hence bypassing those that registered poorly). The concatenation of rigid transformations yielded a reconstructed histological volume. Such reconstruction method was reused in Adler et al. (2014) with different edge weights. Later, however, manual histology reconstructions using HistoloZee were used in Yushkevich et al. (2016) and Adler et al. (2016). While much more labor-intensive, it was found that manual reconstructions led to better histology reconstructions especially when slices were torn or poorly stained. 
Multimodal alignment. Once an initial histological volume is available, a coarse, linear alignment of the geometries of both medical image and histological volumes is performed (Step 2), which may then be refined by non-linear registration (Step 3). This can be achieved using standard inter-modality registration techniques implemented in packages such as AIR (Woods et al., 1998a) followed by Diffeomap (LDDMM) (Li and Mori, 2001) in Ceritoglu et al. (2010), landmark-based registration followed by ANIMAL (Collins and Evans, 1997) in Chakravarty et al. (2006), or maximisation of mutual information (Wells et al., 1996) followed by free-form deformations (Rueckert et al., 1999) and a pyramidal approach with gradually increasing number of control points in Delzescaux et al. (2003). Only global linear registration was performed in Stille et al. (2013).

Variations. A variation of Procedure 1 consists of complementing Step 2 with the registration of every slice of the globally aligned histological volume with its 2D counterpart in the medical image volume. Such variation is the cornerstone of hybrid approaches described in \$5.1.4. Li et al. (2009) aligned the centres of mass of each histological slice with their corresponding in vivo MR plane (although this is not a registration per se) after 3D rigid registration between both volumes. This was refined by $3 \mathrm{D}$ non-rigid registration using the adaptive bases algorithm (ABA) (Rohde et al., 2003). Yushkevich et al. (2006) deformed each histological slice of the 3D aligned histological volume towards the average of its immediate neighbours (predecessor and successor) and the corresponding MR slice. Some of their methods have been included in the framework developed by Majka and Wójcik (2015) ${ }^{10}$. Humm et al. (2003) only performed linear registration between every histological slice and its 2D counterpart in the MR.

Matching in vivo via ex vivo imaging. Ex vivo correspondences can be further carried to in vivo space by an extra registration between both image volumes. Given the registration between histology and ex vivo, concatenating transformations relates histology and in vivo medical imaging. As such, Alic et al. (2011) performed 3D linear (rigid, affine) and elastic registration between histological and ex vivo MR volumes, followed by 3D rigid, affine and elastic registration between ex vivo and in vivo MR volumes. All registrations were performed using Elastix (Klein et al., 2010). Affine registration between the reconstructed histological and ex vivo volumes was also performed using Elastix in Rusu et al. (2015). Ex vivo and in vivo medical images were affinely registered, yielding linear alignment between histology and in vivo. Finally, non-rigid registration between histological and in vivo volumes refined the alignment of both modalities using free-form deformations in a multiscale setting.

\subsubsection{Hybrid methods}

We call hybrid methods those similar to Procedure 1 except that both the serial arrangement of the histology stack (its reconstruction) and its alignment relative to the medical image volume are jointly refined (Procedure 2). Both processes hence benefit from each other as changing one affects the other. Initial histology reconstructions (Step 1) follow methods presented in $§ 5.1 .3$.

Mono/Multimodal registrations. Step 2 consists of iterating over two registrations: (i) a 3D-3D registration, which updates the global alignment between the current estimate of the histological volume and the 3D medical image, and (ii) 2D-2D registrations, which affect the serial arrangement of slices relative to each other by aligning them with their (current) corresponding slice in the medical image volume used. This in turn provides a new histological volume which is used at the next iteration. This process is repeated until convergence. In general, linear registrations are used in the iterative process to avoid creating wrong correspondences through non-rigid registration. In particular, block-matching was used in Malandain et al. (2004), the ANTs toolkit with NMI was used in Adler et al. (2014) and maximisation of MI was used in Yang et al. (2012). In the latter, the specific case of separate pieces of tissue was addressed using 2D piecewise local registration. They also tackled the challenging task of automatically initialising the location of a tissue block that is a sub-volume of the tissue MR. Yang et al. identified the locations of the first and last slices of the histological block in the MR as those maximising NMI after 2D rigid registrations.

Once the iterative process has converged, a final step may consist of a non-rigid registration to refine the matching (Step 3). The 2D diffeomorphic registration in Adler et al. (2014) was similar to that introduced by Yushkevich et al. (2006), while cubic B-spline parametrisation for 3D non-rigid registration and the normalised correlation coefficient was used as a similarity measure in Yang et al. (2012).

\footnotetext{
${ }^{10}$ http://www.3dbar.org/wiki/barPosSupp. The source code is available for download at https://github.com/pmajka/poSSum.
} 


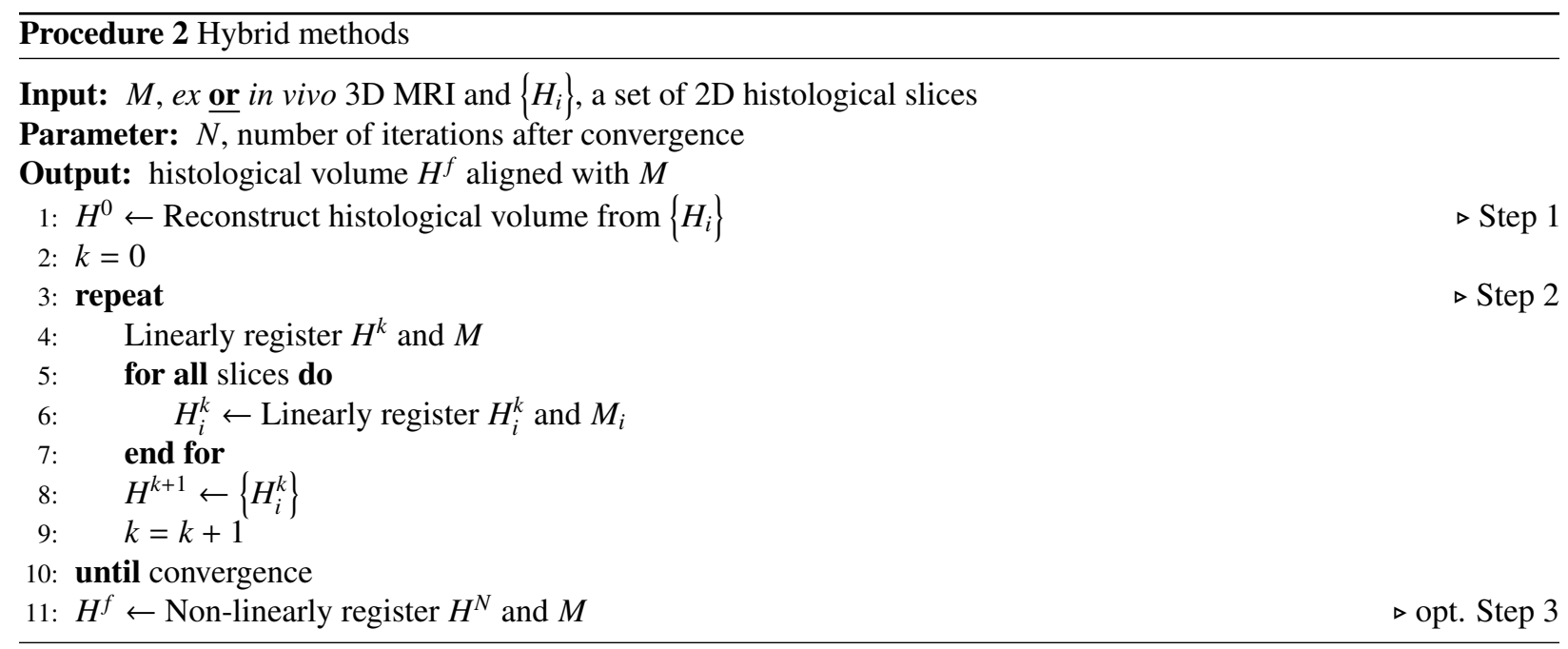

Variations. A variation of Step 2 was proposed by Ou and Davatzikos (2009). They iterated over: (i) a 3D affine registration between the current histological and MR volumes, by maximisation of the correlation coefficient and (ii) a 2D rigid registration between every histological slice and the central histological slice. Subsequent steps jointly addressed the segmentation and the refinement of the registration of prostate cancer images and also consisted of an iterative process.

Another variation was proposed by Goubran et al. (2013), who embedded the non-rigid registration of Step 3 in the iterative process of Step 2. The pipeline thus consisted of iterating over: (i) a 3D rigid registration of the current estimate between the current histological volume with the MR, (ii) 2D rigid registrations between every histological slice and its currently corresponding MR plane, and (iii) 2D non-rigid registrations between every histological slice and its currently corresponding MR plane using free-form deformations.

Matching in vivo via ex vivo imaging. Correspondences between histology and ex vivo can be further carried to in vivo space similarly to \$5.1.3. Goubran et al. (2015) first translated the ex vivo MR to match the in vivo MR space in order to facilitate the placement of landmarks in subsequent steps. Then, 3D linear (rigid+scaling) landmark-based registration was performed between in vivo and ex vivo MRs using manually picked landmarks. This was refined by 3D non-rigid registration between both 3D MRIs using landmark-based registration and a symmetric implementation of FFD, respectively for hippocampal and neocortical specimen.

\subsection{Histology $\leftrightarrow 3 D$ medical imaging using blockface photographs}

Blockface photographs provide structural information of the tissue face prior to cutting and therefore allow correcting for subsequent tissue deformations (mainly induced by cutting, floating and mounting). In theory, they should be inherently aligned by virtue of the set-up: it consists of a camera on a tripod—or mounted on the microtome itself-oriented towards the face of the tissue block secured on the microtome, and which imaging plane is parallel to the block face. Though, it is common to affinely register them with each other in order to account for small displacements (Annese et al., 2006; Yelnik et al., 2007; Groen et al., 2010) but this not robust to perspective distortions (the camera imaging plane is never and can not remain truly parallel to the block face). Eiben et al. (2010) considered that each time the microtome cuts a section out of the tissue block, its face lies in a slightly different plane (due to small mechanical imprecisions or due to the expansion of the tissue when a new face is exposed). This leads to a perspective error, which may hinder the consistency of the reconstructed volume. Their method provided a way to correct for scaling variations and displacements of the sample that may occur from one acquisition to the next. However, they did not take into account the camera motion (as small as it may be). Breen et al. (2005b) assessed the camera lens for image distortion by ensuring that lines from manually selected points (including edges of the image) remained straight. They also ensured those lines were not blurred in any region of the image. Casero et al. (2017) corrected the perspective error of the blockface photograph aquisition by computing a projective transformation using manually 


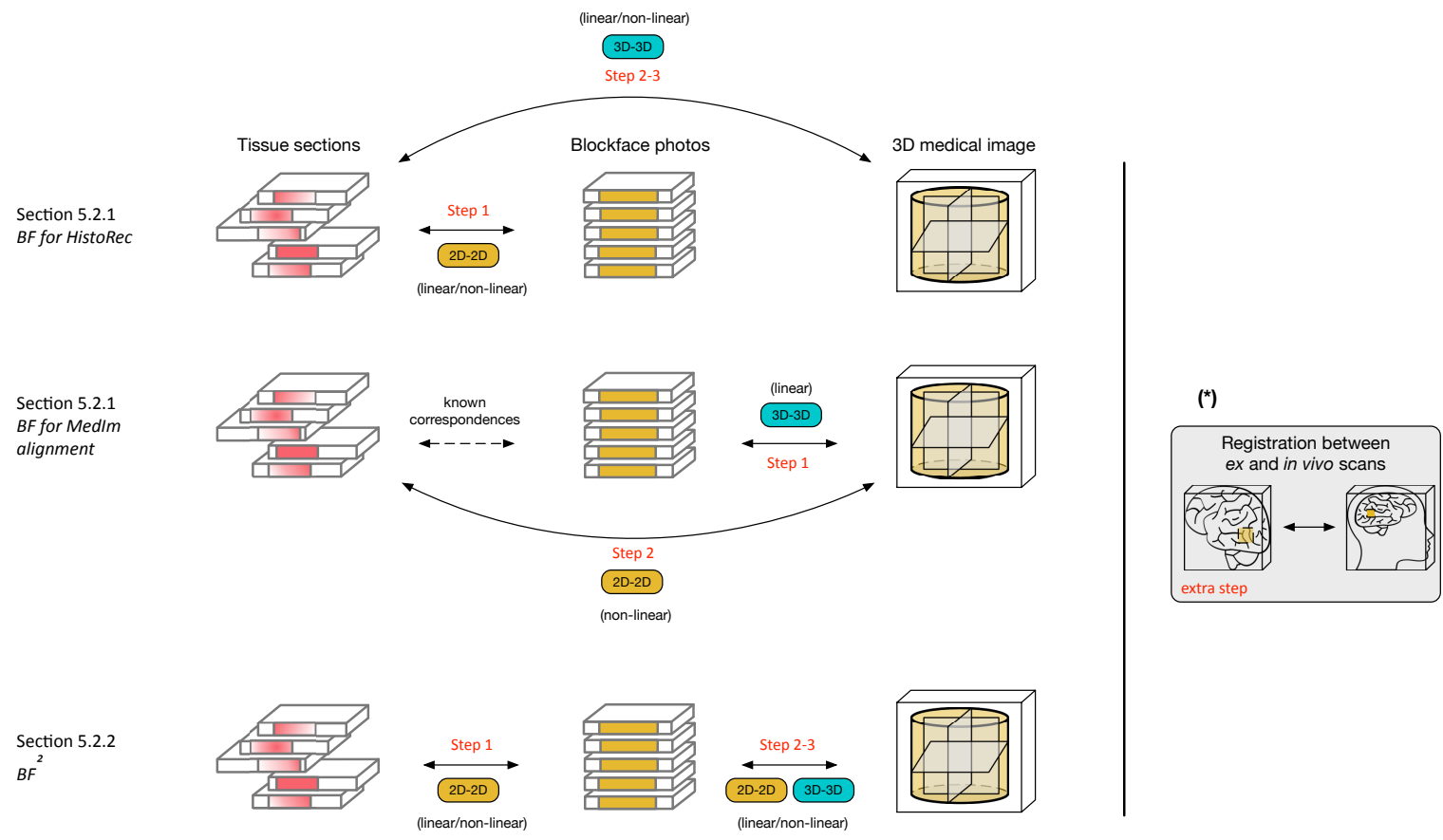

Figure 4: Strategies to register histology with volumetric medical images using blockface photographs as intermediate modality; (*) see Fig. 3.

extracted landmarks. "Scratched" photographs (which occur when using a poor quality knife blade during microtomy) were also taken care of: images were first rotated to make the scratches horizontal/vertical using the wax block sides; then, the image rows/columns intensities were scaled so that their median values equaled the wax median value.

We identified three main types of pipelines that relate histology to volumetric medical imaging according to how they exploit blockface (BF) photographs as an intermediate modality (Fig. 4), namely for histology reconstruction or for alignment with medical imaging (§5.2.1: resp. BF for HistoRec, and BF for MedIm alignment) and for both (§5.2.2: $\left.\mathrm{BF}^{2}\right)$.

\subsubsection{Single use of blockface photographs}

These pipelines use the known correspondences between each histological slice and a blockface photograph for reconstruction, and follow Procedure 3.

\section{Procedure 3 BF for HistoRec}

Input: $M$, ex or in vivo 3D MRI, $\left\{H_{i}\right\}$, a set of $2 \mathrm{D}$ histological slices, $B=\left\{B_{i}\right\}$, a set of $2 \mathrm{D}$ blockface photographs. Output: histological volume $H$ aligned with $M$

1: for all slices do $\triangleright$ Step 1

2: $\quad H_{i}^{0} \leftarrow$ Register $H_{i}$ and $B_{i}$

3: end for

4: $H^{0} \leftarrow\left\{H_{i}^{0}\right\}$

5: $H^{1} \leftarrow$ Register $H^{0}$ and $M$

$\triangleright$ Step 2

6: $H^{f} \leftarrow$ Non-linearly register $H^{1}$ and $M$ $\triangleright$ opt. Step 3

In Step 1, Alegro et al. (2016) affinely registered each histological image with its corresponding blockface photograph by optimisation of mutual information, as defined by Mattes et al. (2003). Schormann and Zilles (1998) first reconstructed the blockface volume by least square minimisation between corresponding pairs of landmarks in adjacent 
photographs (Schormann et al., 1995) and then registered each histological section with its corresponding blockface photographs using an extension of principal axes theory generalised to affine transformations (in order to be able to account for shearing artefacts introduced during the tissue preparation). Johnson et al. (2010) used 2D moments-based rigid alignment for some brains, then refined using AIR software. For other brains, the method by Thevenaz et al. (1998) was used between corresponding images with manual refinement. This protocol was reused in Johnson et al. (2012).

In Steps 2 and 3, Alegro et al. (2016) used symmetric diffeomorphic 3D registration, SyN (Avants et al., 2008) to align the reconstructed histological and the MRI volumes. Johnson et al. (2010) first linearly aligned the reconstructed histological with MR volumes using a quaternion transform followed by an affine transform. Then, the alignment was refined using a multi-resolution diffeomorphic registration algorithm (Avants et al., 2008). Schormann and Zilles (1998) first performed a 3D affine registration between the histological and the MR volume, followed by a 2D non-linear registration between every histological sections and its corresponding MR plane using a 3D elastic full-multigrid technique (Stüben and Trottenberg, 1982) restricted to 2D (Rohr et al., 1996). It was initialised with a 2D linear registration driven by the analysis of Rayleigh-Bessel statistics, which describe the probability density of local non-linear deformations in histological sections (Schormann et al., 1995).

Variations. A variation of Step 2 consists of considering slices instead of volumes: in the method developed by Bürgel et al. (1999), after reconstructing the histological volume, every histological slice was warped to its corresponding ex vivo MR plane, based on the methodology developed in Schormann et al. (1993, 1995).

A variation of Procedure 3, BF for MedIm alignment, was proposed in Amunts et al. (2013) and relied on the registration between the blockface volume and the $3 \mathrm{D}$ medical image. Then, using the direct, known $2 \mathrm{D}$ correspondences between histology and blockface, every histological slice was registered with its corresponding MR plane.

\subsubsection{Dual use of blockface photographs}

These pipelines use blockface photographs for both histology reconstruction and matching with 3D medical images. They are to be the most frequent way to address the problem and follow Procedure 4 .

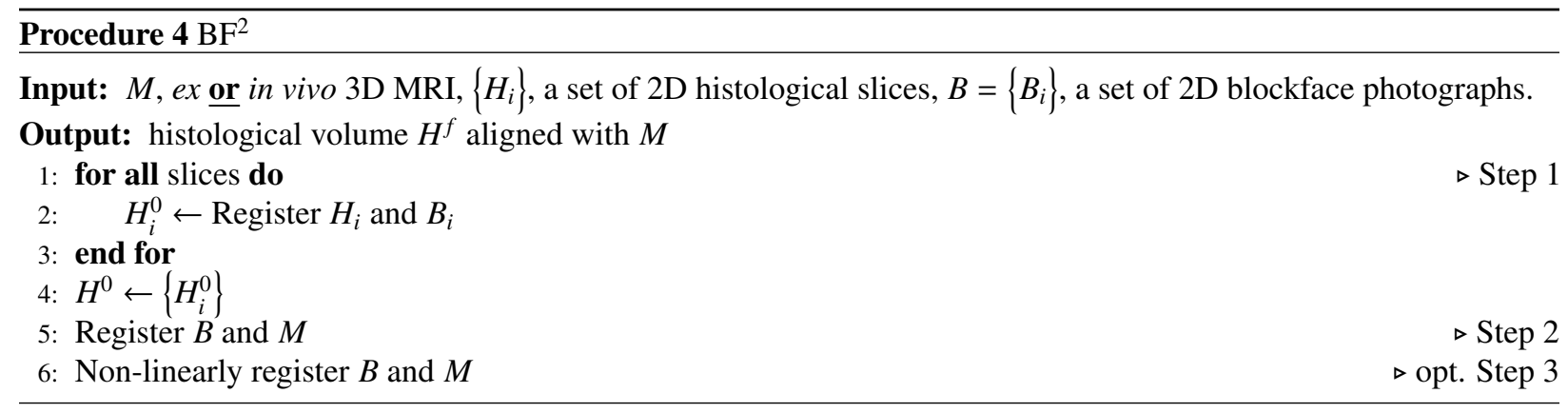

Linear transformations. The choice of aligning every histological slice to its corresponding blockface photograph (Step 1) using linear registration (as opposed to non-linear) may stem from the poor content that the unstained tissue face exhibits. Blockface photographs provide little structural information apart from the tissue borders (higher contrast with the surrounding embedding medium), which could lead to erroneous deformations of the inside of the tissue. Linearly registering every pair of corresponding images may suffice to restore a globally consistent arrangement of histological slices, which will be refined locally when matched with the medical image volume. Lebenberg et al. (2010) rigidly aligned every histological section with its corresponding blockface photograph; every autoradiograph (see "Cerebral function" in $\S 6$ for a brief definition of autoradiography) was then rigidly registered with its histological slice counterpart, both using block-matching (Ourselin et al., 2000). This was reused in Vandenberghe et al. (2016). Yelnik et al. (2007) first reconstructed the blockface volumes using ICP i.e., by registering every photograph to its immediate neighbour (binary images of segmented rivets) and then performed 2D rigid registration between every histological section and its cryo-blockface counterpart. In order to refine the histological volume, 3D regions of interest 
centred around the basal ganglia were extracted using Yav++ software (Delingette et al., 2001) in both histological and blockface volumes and 2D hierarchical registration (rigid, homothetic and affine) was performed between corresponding 2D images. Dauguet et al. (2007b) aligned every histological section with its corresponding blockface photograph using a "hemi-rigid" method, which accounted for the independent spreading of the hemispheres on the glass slides.

Non-linear transformations. Other authors have chosen to refine Step 1 with non-rigid registration. For example, Choe et al. (2011) performed 2D linear followed by 2D non-linear registrations between light micrographs and corresponding blockface photographs using respectively maximisation of MI and ABA (Rohde et al., 2003). Breen et al. (2005b) performed 2D non-rigid registration between a histological section and its corresponding blockface photograph using TPS and corresponding landmarks: interior ink fiducials and anatomical landmarks, such as blood vessels, and corresponding points along the external tissue boundary using "live-wire" semi-automated algorithm (Falcão et al., 1998; Mortensen et al., 1992). TPS was also used in Meyer et al. (2006) with 7 control points; six control points were used in Piert et al. (2009) and Park et al. (2008). Groen et al. (2010) performed 2D rigid, followed by 2D non-rigid FFD-based registrations (Rueckert et al., 1999).

In Steps 2 and 3-consisting of registering blockface and medical imaging-pipelines start with 3D linear registration (Groen et al., 2010). Dauguet et al. (2007b) performed 3D rigid registration between the blockface volume and the in vivo T1 MRI by maximisation of mutual information, as defined by Viola and Wells III (1997). 3D non-rigid registration between the blockface volume and the MRI was performed using free form deformations, similarly to what Groen et al. (2010) proposed, and the composition of the two previous transformations was applied to the "hemi-rigid" transformed histological volume. The same sequence was applied by Lebenberg et al. (2010). Choe et al. (2011) also described a similar strategy apart from the last step of the non-rigid registration between blockface and ex vivo T2w MR volumes, for which they made use of ABA. The composition of the transformations between blockface and the medical image volume, and between histology and blockface volume was applied to the T2w image in order to resample it in the histological space - this strategy was reused by Schilling et al. (2018) to study the correspondence between diffusion MRI measures and realistic biological fiber architectures. Meyer et al. (2006) performed 3D non-rigid registration between blockface and ex vivo MR volumes using TPS with 6 control points; 18 control points were used in Piert et al. (2009) and Park et al. (2008).

Other applications of Procedure 4 without the non-rigid refinement of Step 3 include Yelnik et al. (2007), who performed 3D rigid registration between blockface and ex vivo T1w MR volumes one hemisphere at a time (Prima et al., 2002). The alignment was refined by a 3D hierarchical registration between $3 \mathrm{D}$ regions of interest centred around the basal ganglia and propagated to the full volumes. Breen et al. (2005b) performed 3D linear (rigid+scaling) registration between the blockface volume (stack of 3mm-thick slice faces) and the MR volume, for which the centres of the needle paths were manually segmented. The global transformation was optimised using the ICP algorithm. Non-rigid refinement was not performed and the method was validated in Lazebnik et al. (2003).

Variations. A variation of Procedure 4 was proposed by Uberti et al. (2009) and consists, as in §5.2.1, of considering slices instead of volumes. After reconstructing the histological volume (Step 1), 2D non-registration was performed between every blockface photograph and the corresponding in vivo MR planes. Registrations were based on moving landmarks sampled on curves generated from the contours of corresponding anatomical features. Once landmarks locations were optimised for matching through minimisation of a cost function based on the local curvature of the curves and limited to small displacements, TPS interpolation for point-based registration was performed.

Matching in vivo via ex vivo imaging. Ex vivo correspondences can be further carried to in vivo space through an extra registration between $e x$ and in vivo medical image volumes in order to relate histology to in vivo imaging. As such, Groen et al. (2010) rigidly (point-based) registered the ex vivo $\mu \mathrm{CT}$ and the in vivo CTA using manually selected landmarks (e.g., calcium spots, lumen, bifurcation position are clearly visible in both medical imaging modalities). Meyer et al. (2006) performed 3D non-rigid registration between the ex vivo and in vivo MRs using 3D TPS with 14 control points, and it was used as an initialisation for a last non-rigid registration between in vivo volume and the blockface image by optimising the position of 7 control points and using mutual information as the objective function. Piert et al. (2009) reused the methodology presented in Park et al. (2008): the ex vivo MR and in vivo T2 MR volumes were non-rigidly registered using TPS with 7 control points. The T2 MRI was chosen as the reference space. 
Additionally, in vivo T2 and CT volumes were non-rigidly registered using TPS with 7 control points and PET and CT volumes were rigidly registered.

\section{Validation methods}

We hereafter detail the ways authors have validated the accuracy and the precision of image registration, as defined by Maintz and Viergever (1998), in the context of histology reconstruction (with or without the help of medical imaging).

Visual assessment. It may be the most intuitive way of validating the registration accuracy but must be carried out by experts and does not provide with any quantitative measure. In the case of histology reconstruction without the help of 3D medical images, criteria used to tell whether registrations are successful include visually improved representations of small structures (subcortical nuclei, cortical areas) and smooth inner and outer borders (Wirtz et al., 2004). Wirtz et al. used three classes of neuroanatomical structures that are recognisable after registration when examining whole rat brains: subcortical nuclei, ventricles, certain cerebral and cerebellar cytoarchitectonic layers. Smoothness was explicitly used a criterion for reconstruction in Cifor et al. (2011). Ju et al. (2006) compared the reconstructed volume to real histology sections from the Paxino's Atlas (Paxinos et al., 2000) at similar sagittal and horizontal locations.

Visual assessment can also be used when comparing one method against others (assuming that the same data have been used). Gaffling et al. (2011) compared the reconstruction against that obtained through standard non-registration scheme (without landmarks) using histological data from Ju et al. (2006) and artificial data. Ju et al. (2006) compared the reconstruction against that obtained from the method described in Guest and Baldock (1995).

When medial imaging is available, visual assessment can be performed by (i) cross-section comparison. Malandain et al. (2004) used two synchronised 3D viewers to display the two volumes in the same geometry. This allowed showing same cross-sections (axial, sagittal and coronal) as well as a cursor at corresponding positions. Alic et al. (2011) qualitatively evaluated the alignment between 3D in vivo $\mathrm{T} 2$ and histological volumes with two observers using visual inspection with a moving quadrant view; or by (ii) superposition of adjacent sections (Li et al., 2009; Choe et al., 2011). Dauguet et al. (2007b) superimposed the external and internal borders of the MRI brain-extracted using Deriche filter (Deriche, 1987) - registered onto the blockface volume. A similar visulisation was used to assess the quality of the histology reconstruction in Lebenberg et al. (2010); Malandain et al. (2004); Colchester et al. (2000).

Landmark-based validation. It is the most widespread method, used for example in Nir et al. (2014); Gibson et al. (2012); Liu et al. (2012); Ward et al. (2012); Yang et al. (2012); Ou and Davatzikos (2009). It consists of computing the Euclidean norm between corresponding tie points extracted in two images (also referred to as target registration error, TRE). This measure might not be not appropriate for the validation of histology reconstruction from 2D sections only (Ju et al., 2006): a minimum distance does not mean the true shape has been recovered. It is however very relevant in the case of multimodal registration. Those landmarks can be:

- needle tracks, such as in Colchester et al. (2000); Lazebnik et al. (2003); Breen et al. (2003).

- manually identified anatomical landmarks, that are visually tractable across modalities. The anterior commissure, the pillars of fornix, perivascular spaces and optic chiasm were used by Kim et al. (2000). Automatically extracted sulcal lines of maximal depth (sulcal fundi or sulcal bottom lines) were used by Osechinskiy and Kruggel (2010); the extraction procedure is described by Lohmann (1998). The urethra, nodules, scars (from previous biopsies), calcifications, and "other general distinguished anatomical features" were considered by Nir et al. (2014) with the help of a radiologist. Landmark points were extracted manually by Goubran et al. $(2013,2015)$ to compute the TRE. Boundary curves were manually drawn by Adler et al. (2014) to compute the boundary displacement error. When such landmarks are used for registration, reliability of their locations is usually assessed by looking at intra- and inter-user variability. For example, Gangolli et al. (2017) evaluated the former by asking a user to perform landmark selection twice, five days apart, and comparing registered voxels shifts. The latter was assessed by asking two different users to perform the previous procedure. In addition, artificial perturbation of an established set of landmarks in histology was performed in order to test the robustness of the registration method to such changes.

- anatomical artefacts. Singh et al. (2008) used the centroids of manually segmented lesions to evaluate and validate the registration accuracy. Alic et al. (2011) identified characteristic features in the tumour and its contour. 
- Ink marks made on the fresh tissue, such as in Breen et al. (2005b).

Measures of overlap. They rely on regions of interest (RoIs) manually delineated by an expert in two images. The Dice score or the Jaccard index are two measures that can be computed to quantify the amount of overlap between the two regions (Li et al., 2009; Alegro et al., 2016; Beare et al., 2008; Hess et al., 1998; Baheerathan et al., 1998; Nagara et al., 2017). Specifically, Lebenberg et al. (2010) manually delineated the hippocampus, cortex, and striatum, as well as the corpus callosum and substantia nigra, to compare different reconstructed histology volumes. The hippocampus was also manually delineated in every histological atlas in Palm et al. (2010). In Mazaheri et al. (2010), the whole prostate, the peripheral zone, and the transition zone were outlined by an experienced radiologist in MR and histology images. Nir et al. (2014) compared the segmentations in the registered histological sections with the corresponding manual segmentations in the re-sliced images of the MR volume of the prostate. The Dice score was shown to be a reliable indicator of registration accuracy only for small and localised RoIs (which approximate point landmarks) in several locations in the image space (Rohlfing, 2012).

Texture-based methods. Grey-level co-occurrence matrices (GLCM) were presented in Haralick (1979) and were used to assess the quality of the histology reconstruction in Baheerathan et al. (1998); Cifor et al. (2011). Such matrices were computed by calculating how often the pair made of a pixel of interest with a certain intensity and its immediate neighbour in the direction going across slices (orthogonal to the cutting plane) occurs. Cifor et al. (2011) computed GLCMs in the neighbourhood around the boundaries of the tissue rather than the whole volume in order to quantify the smoothness of the reconstruction.

Artificial perturbation of a ground truth. Artificially perturbing a ground truth allows having access to the original alignment, against which the resulting alignment is compared. It is done by taking a volumetric image which original alignment is known (e.g., a 3D medical image) and applying random, smooth transformations to each of its slice independently (as well as e.g., including artefacts that simulate holes/tears, ignoring some slices etc.). The error made after reconstruction is then computed (Cifor et al., 2011; Nikou et al., 2003; Ju et al., 2006; Braumann et al., 2005; Majka and Wójcik, 2015; Bagci and Bai, 2010). Synthetic datasets (e.g., phantom models) were used in Schwier et al. (2013); Ou and Davatzikos (2009); Arganda-Carreras et al. (2010). Comparison against manually realigned stack (by an expert physician researcher) can also be performed (Krinidis et al., 2003a; Groen et al., 2010). Robustness to holes and tears was tested by Cifor et al. (2011), and the effect of missing sections was assessed by Arganda-Carreras et al. (2010). Both were addressed by Nikou et al. (2003).

\section{Applications}

We underline three main areas of applications within which the covered literature falls into: (i) examining structures with respect to their environment in $3 \mathrm{D}(\$ 7.1)$ with or without the help of 3D medical imaging; (ii) the correlation of data (§7.2), which benefits from the access to the underlying microbiology to improve the characterisation/discrimination of signals in non-invasive imaging; and (iii) the creation of digital atlases (\$7.3), which allows for easy 2D and 3D visualisations as well as quantitative measures of anatomy when independent data from different subjects are included.

\subsection{Examining functions and relationships in $3 D$}

Organs. Mice brains were reconstructed in Gaffling et al. (2011, 2015) and Müller et al. (2014). Nissl-stained cryosections of an adult mouse brain, available from Ju et al. (2006), were used in Gaffling et al. (2011). Rat liver tissues were studied by Schwier et al. (2013), who proposed a registration method for the reconstruction of histological whole slide images that exhibit vessel structures. Human liver tissues were studied by Song et al. (2013) and Roberts et al. (2012). Chen et al. (2003) described the 3D configuration of extravascular matrix patterns in archival human uveal melanoma tissue. Mice lungs (Rusu et al., 2015) and heart (Magee et al., 2015; Mansoori et al., 2007) were also studied.

Tumours. The 3D structure of tumoural invasion fronts of carcinoma of the uterine cervix was investigated by Braumann et al. (2005) in order to understand their architectural-functional relationship, while Wentzensen et al. (2007) analysed the spatial organisation of a cervical cancer. 
Vasculature. Xu et al. (2015) studied the microvascular structure of the mouse hind-limb. Due to the 3D rearrangement of the microvessel networks during pathology, a reconstruction was critical in understanding the dysfunction of organs during disease. The 3D vascular network from immunostained sections of the human spleen was reconstructed by Ulrich et al. (2014).

Cerebral function. It is dependant on neurological organisation and metabolic activity (Hibbard et al., 1987); autoradiography allows looking in great details at, among others, the cerebral metabolic rate of glucose utilisation in response to physiologic activation of the visual, auditory, somatosensory, and motor systems, and in pathologic conditions. Rat brains are often studied (Hibbard and Hawkins, 1984, 1988; Zhao et al., 1993; Kim et al., 1997, 1995; Bronchti et al., 2002; Nikou et al., 2003; Lee et al., 2005; Dubois et al., 2007). Bronchti et al. (2002) studied the auditory activation of visual cortical areas in the blind mole rat. Lee et al. (2005) studied the cerebral glucose metabolism in the rat cortical deafness model using 3D voxel-based statistical analysis of autoradiographic data. They observed a significant decrease in the glucose metabolism in the bilateral auditory cortices. Dubois et al. (2007) combined histology and autoradiography to study interhemespheric differences through voxel-wise statistical analyses. Hess et al. (1998) studied the metabolism and function of gerbil brains. Autoradiographic volumes from 2-DG autoradiographs of primates were reconstructed using 3D MRI (Rangarajan et al., 1997; Malandain et al., 2004).

\subsection{Characterising $3 D$ medical imaging signals}

Neurological diseases. A 3D mapping of pathological changes throughout the brain for Creutzfeld-Jacob disease was developed by Colchester et al. (2000). Goubran et al. (2013, 2015) identified and delineated lesions in MRI in order to improve the surgical treatment of epilepsy. Lockwood-Estrin et al. (2012) investigated specific semi-quantitative 3T MRI parameters in order to understand whether they are associated with particular histological features. The study was performed on temporal lobe specimens in epilepsy surgery patients whose conventional MRI scan appeared normal.

Stroke. Li et al. (2006) correlated signal changes observed in T1-weighted images acquired during brain ischemia in small animal models to molecular features obtained from histology. A similar effort was followed by Stille et al. (2013), who registered "abnormal" images from a rat model of stroke with 3D in vivo T2w MR images to study neurobiological correlates of the variations in MRI signal intensities.

Cancer. Histopathological examination can be related to in vivo-or ex vivo (Gibson et al., 2012), MR imaging with the aim of improving prostate cancer detection rate (Nir et al., 2014; Ward et al., 2012; Alic et al., 2011; Chappelow et al., 2011a; Samavati et al., 2011; Xiao et al., 2011; Mazaheri et al., 2010; Ou and Davatzikos, 2009; Zhan et al., 2007). Le Nobin et al. (2015) compared prostate tumour boundaries on MRI to those in histology in order to define an optimal treatment margin for achieving complete tumour destruction during image guided focal ablation. Edwards et al. (2005) used histology to identify the tumour boundaries in oral cancer patients with better accuracy in order to enable precise PET-guided resection. Jiang et al. (2013) combined in vivo MRI/MRSI, ex vivo brightfield/fluorescence microscopic imaging, and histology to study human breast cancer. Seeley et al. (2014) studied secondary breast cancer in the bone using diffusion weighted MRI, Matrix-Assisted Laser Desorption/Ionisation Imaging Mass Spectrometry and histology in order to observe changes caused by tumour cells in the bone at the protein level. Studies from Mertzanidou et al. $(2017,2016)$ investigated the mapping between histology and 3D whole specimen imaging along with whole mastectomy volume reconstruction from radiographs. Breen et al. (2005b) correlated in vivo MR thermal lesion images with histological tissue damage in rabbit thighs. Humm et al. (2003) developed a stereotactic fiduciary marker system for hypopharyngeal tumour xenografts in rodents to co-register MRI, PET, histology, autoradiography, and measurements from physiologic probes.

Vascular lesions. can be seen in human MRI but are only detected reliably in histology. Singh et al. (2008) registered lesions microscopical features with their corresponding locations in the in vivo MR images in order to understand better their MRI signatures. Coombs et al. (2001) correlated MR signal characteristics with carotid atherosclerosis plaque components in order to optimise protocols for future clinical carotid MRI. Groen et al. (2010) studied the relationship between biomechanical parameters and atherosclerotic tissue components in the carotid using histology, in vivo CT angiography and ex vivo MRI and CT imaging. 


\subsection{Combined MRI-histology atlases}

Atlases provide detailed segmentations and classifications of certain regions and sub-regions in a common anatomical reference framework. They stem from the need for accurate maps of architectonic areas with reference to MRI images. The main rationale is to help understanding the localisation of functional activity in different regions (Brett et al., 2002) but they are also of great importance in segmentation (Aljabar et al., 2009) or can be used to improve preoperative planning (Goerres et al., 2017) and post-operative follow-up.

There exist three types of atlases:

- MRI-based atlases (Kovačević et al., 2005; Dorr et al., 2008). They are useful for measuring volumes and analysing large morphological features but suffer from imprecise delineations due to low resolutions.

- Histology-based atlases (Ju et al., 2006). Most of them derive from rodent brains but are limited: it was reported in Ma et al. (2005) that distortions during tissue preparation and the lack of structural ground-truth in 3D make it hard to extract spatial cues or to derive quantitative group variations. Annese et al. (2006) proposed a high-resolution 3D reconstruction of blockface-imaged Methylene blue perfused primate brain tissue as the basis for detailed stereotaxic anatomical atlases. The use of blockface images bypasses the tedious correction and alignment of histological sections without external information.

- Combined MRI and histology atlases (Saleem and Logothetis, 2012). They combine accurate anatomical delineations in histology for propagation in the 3D medical image, with ground truth 3D shape for improved histology reconstructions. We emphasize this last type of atlases as it involves multimodal image registration.

Human studies. The creation of a brain atlas of the human basal ganglia based on histological images and MR images was investigated by Ourselin et al. (2001a). Other brain atlases of the basal ganglia and the thalamus were then proposed by Chakravarty et al. (2006), Yelnik et al. (2007), and Bardinet et al. (2009) based on immunohistochemical and MRI data.

Animal studies. Johnson et al. $(2007,2010)$ presented an atlas of the C57BL/6 mouse brain based on MRI and conventional Nissl histology. Lebenberg et al. (2010) derived an MRI-based 3D digital atlas from C57Bi/6J mouse brain, which was matched with $3 \mathrm{D}$ reconstructed post mortem data in order to evaluate morphology and functions of mouse brain structures in the context of Alzheimer's disease. Mailly et al. (2010) reported a procedure for the construction of a 3D digital model of the primate and rodent basal ganglia. Yushkevich et al. (2006) used an average MRI of 30 in vivo scans of 10 mice in order to build a 3D reference histological atlas of the mouse brain from Nissl-stained sections. Ali and Cohen (1998) combined histological sections of rat brain with a 3D brain atlas in order to contribute to brain mapping efforts. Gefen et al. (2008) followed the same path by aligning Nissl-stained histological sections with a volumetric mouse brain atlas for the segmentation of hippocampal complex. Dauguet et al. (2007b) proposed a pipeline for the reconstruction of a histological volume from whole baboon brain Nissl-stained sections using in vivo MRI. Amunts et al. (2013) created an ultrahigh-resolution 3D model of a human brain at nearly cellular resolution of $20 \mu \mathrm{m}$. Burton et al. (2006) combined MRI and Trichrome-stained histological sections in order to construct histo-anatomically detailed models of cardiac 3D structure and function at a high resolution.

\section{Discussion and perspectives}

This section covers three topics: (\$8.1) some methodological comments on pipelines, their differences, advantages and drawbacks; (§8.2) some of the remaining challenges; and (\$8.3) concluding remarks on the importance of crossdisciplinary knowledge in solving the biological question associated with histology-MRI registration. Note that the discussion is directed towards the multimodal correspondence problem that underpins $3 \mathrm{D}$ histology reconstruction with the help of 3D medical imaging.

\subsection{Methodological comments}

On hybrid pipelines. We comment on three points: (i) the methodology per se, (ii) the usefulness of complex initial 3D histology reconstructions when medical images are available and (iii) the types of datasets and what to do with them.

(i) Volume-based approaches $(\$ 5.1 .3)$ rely on a single round of registration between histology and medical image volumes. In that respect, changing the input (another reconstructed histology volume) results in a different global 
alignment and thereby establishes different correspondences between both modalities. On the contrary, hybrid pipelines (§5.1.4) rely on the principle that global alignment between both volumes is optimal only when the arrangement of histological sections relative to each other (the 3D histology reconstruction) is itself optimal, and vice versa. In other words, one conditions the performance of the other. This strategy offers a robust way to achieve accurate reconstructions and we highlight two representative works from Adler et al. (2014) and Goubran et al. (2013):

- Goubran et al. relied on an iterative process that consisted of both (3D and 2D) linear and (2D) non-linear registrations between histology and medical imaging. However, there is no preliminary guarantee that a histological section and its "corresponding" (resampled) MRI plane at some iteration are actual counterparts to each other. In fact, unless the histology stack has first been well initialised (with a linear transformation that brings it to the MRI space), there is a risk of biasing both its final reconstruction and the global alignment with medical imaging by non-linearly transforming histology when registering it with the wrong (resampled) MRI plane.

- On the contrary, Adler et al. performed 2D non-rigid registrations between histology and medical imaging only after the same type of iterative process (though consisting exclusively of linear registrations) had converged.

(ii) In view of Figure 9 from the work by Adler et al. (2014), the gain from complex methods (compared to pairwise registrations or simple stacking) for initial histology volumes when medical images are available is also unclear in general. Furthermore, whereas consistency and accuracy of the initial reconstruction would matter in the case of volume-based approaches, hybrid pipelines allow to relax that constraint as they account for it by design. Manual reconstructions using open-source softwares represent another attractive solution (Yushkevich et al., 2016; Adler et al., 2016).

(iii) It goes without saying that volume-based and hybrid approaches ( $\$ 5.1 .3$ and 5.1.4) as well as pipelines described in $§ 5.2$ all manipulate volumes i.e., assume that a suitable set of histological sections is available. Yet, it happens that one only has access to a single or a few (either sparse, or thin and consecutive) sections, which naturally points towards slice-based approaches ( $\$ 5.1 .1$ and 5.1.2) and no longer aims for histology reconstruction-the correspondence problem solved by multimodal registration remains the same. Now it should be highlighted that every approach that has been described here is driven by the data, which is to be generated in relation to the study and its goals; among the parameters that matter for registration, one should consider the number of and spacing between histological sections relative to the medical image resolution. For example:

- Thick MRI slices relative to a thin slab of several consecutive histology sections (spanning less than one MRI voxel in $z$ ) could either call for 2D-3D or 2D-2D registration techniques in order to establish correspondences; sources of error could come from the identification of the (visually) corresponding MRI plane (the initialisation in the former technique) and from the fact that they rely on interpolation (resampling the medical image volume is more or less effective/meaningful depending upon its resolution).

- A sparse histological dataset, in the sense that its sections exhibit large morphological changes and therefore break the smoothness assumption, may also require to consider them individually (and echoes the previous point).

On the use of intermediate modalities. Mapping histology with medical imaging is challenging due to the alteration of the tissue between the starting (in or ex vivo) and the end (histological images) points of its handling. Not using any proxy may complicate the path to a plausible solution.

Similarly to longitudinal image analysis, the more snapshots during tissue preparation, the easier it is to track and correct deformations between in vivo imaging and sectioned histology (of the same specimen). This is where blockface photographs are of great value (Fig. 5). Using in addition fixed ex vivo medical images allows accounting for the non-uniform shrinkage that happens when extracting the sample. Besides, the main rationale for also using fresh $e x$ vivo scans may be the study of the influence of fixation on tissues magnetic properties (see $\S 8.3$ ). The right balance should however be found when using intermediate modalities as this not only calls for more resources (time, space, study goals etc.), but also impacts the accuracy of the histology reconstruction: (i) none (Adler et al., 2014); (ii) ex vivo medical images (Goubran et al., 2013); (iii) fresh + fixed ex vivo and blockface photographs (Samavati et al., 2011).

Another advantage of intermediate modalities lies in that they offer additional levels of resolutions between that of in vivo imaging and histology. This naturally results in pyramidal schemes, in which the optimisation of the transformation parameters is less likely to get trapped in local optima. 


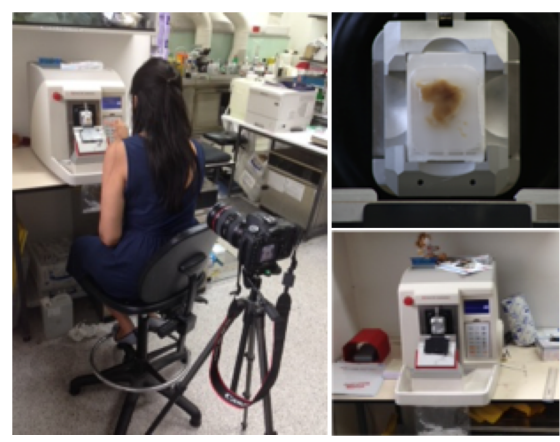

Figure 5: Working with blockface photographs. Left: camera fixed on a tripod, standing behind the histopathologist collecting tissue sections from an automated microtome (bottom right). Top right: one blockface photograph is shown.

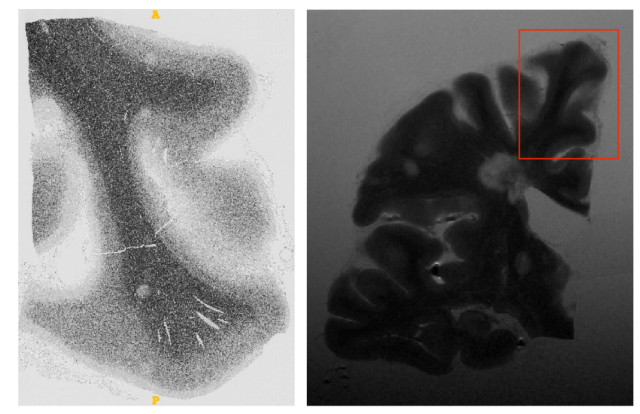

Figure 6: Part-to-whole registration. One T2 slice from a slab of a whole brain is shown in the image on the right. It was then cut into blocks, each of which was put in separate cassettes and processed for histology. A GFAP-stained section from one of these blocks (delineated in red) is shown in the image on the left.

\subsection{Remaining challenges in histology reconstruction}

Preprocessing. Tears and folds may be the most challenging artefacts to account for and frequently result in discarding the damaged section. The correction of tears requires to ascertain that no tissue material has been lost. Assuming this is possible to tell automatically, the problem is to recover in-plane continuity between separated structures. The problem of tears can be extended to purposeful cuts when for example, whole mount histology is to be studied and the tissue slice needs to be cut into several pieces. Mosaicing/stitching is extensively studied in the general computer vision literature (Brown and Lowe, 2007) and may also be approached as Jigsaw puzzle solving (Gallagher, 2012; Noroozi and Favaro, 2016). So far, detection of folds has relied on rather simplistic assumptions about the colour brightness properties of an image and could benefit from the addition of geometrical considerations. It is challenging to know how many layers a single fold is made of. Assuming it is possible to know that number, the correction of a fold still requires the separation of structures belonging to each of the layers that compose it. It also necessitates the rearrangement of the entire piece of tissue according to its configuration prior to folding (i.e., unfolding), which may be approximated from adjacent sections.

Other fields are involved in similar problems and give potential to exciting parallels. They include computational geometry (Demaine and O'Rourke, 2005) and computer vision with e.g., the simulation and visualisation of realistic tearing and cracking of thin sheets (Pfaff et al., 2014); material science with e.g., the study of mechanical instabilities of certain materials during compression (Kim et al., 2011; Pocivavsek et al., 2008); statistical physics with $e . g$., the modeling of folding of thin sheets (Deboeuf et al., 2013; Adda-Bedia et al., 2010); or even geology with $e . g$., the study of orogeny (Ramsay, 1962). Incidentally, extensive information about the nature of tissue distortions during microtomy was already given in Dempster (1942).

Image analysis. The extraction and manipulation of meaningful information from histology and medical images is a very complicated task. Thus, attention has been directed towards simplifying them, that is classically, using the shape 


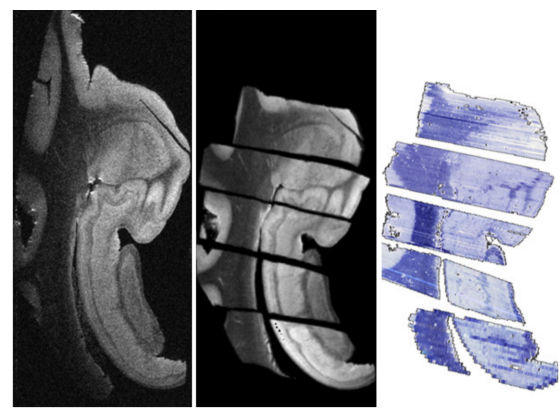

Figure 7: The block of tissue (left) being too thick for histology processing, was cut into sub-blocks, re-scanned (middle) and individually processed. Nissl-stained reconstructed and rearranged histological sub-blocks are shown (right). Image reproduced with permission from Adler et al. (2014).

and the edges.

Such a simplification is not trivial (Marr, 1982) and it cannot be achieved by only looking at intensity changes (Guichard et al., 2004): many unwanted edges are produced in the process, and not only texture and noise are responsible for it (e.g., tears and cuts in histological images). The reduction of images to their main features thus relies on smoothing, which has become a commonplace to separate "true edges" from noise. However, the type of smoothing in multi-scale approaches - how to actually build a scale space? (Morel and Yu, 2011); see also the introduction of Alcantarilla et al. (2012) — remains an important matter to guarantee reliable and tractable detections.

In the monomodal case, feature-based based methods were successfully applied to the registration of histological sections but investigations such as the study of factors that influence keypoint stability in scale space (Rey-Otero et al., 2015), or the derivation of criteria for detectors comparison (Rey-Otero and Delbracio, 2015) may be of great benefit to the field: in general, little details are given about the consistency and reliability of detections. Besides, a common drawback in their use for histopathological image analysis is the large amount of features that may be generated; this is due to the rich content of images, which can quickly turn into "biological noise".

In the multimodal case, histology and medical imaging have, by nature, their own contrast and there does not always exist a mapping between their constituents; incidentally, the latter is one reason why intensity-based methods tend to get trapped in local optima. Geometric approaches rely on a meaningful subset of an image pixels by presupposing that there exists a group of characteristic landmarks which is (relatively) consistent across modalities i.e., which serves the purpose of correspondence; and this is an equally complex task: not only extracting reliable points is difficult (Sotiras et al., 2013), but their description is also very challenging (the purpose of multimodal imaging being to combine images of different nature). As such, classical feature description methods, such as SIFT, will also fail to match features (Toews et al., 2013) especially due to non-linear local intensity mappings (letting aside the lack of counterpart, which could be handled by suitable descriptions and filtering out false matches). Alternative methods are necessary and rethinking descriptions may be required (Heinrich et al., 2012). Note that manual extraction of anatomical landmarks in histology and medical imaging still remains a safe way to establish correspondences (Gangolli et al., 2017). Lastly, shape - as defined by Attneave (1954), can become a valuable asset (see Fig. 6), and multimodal registration may be obtained as a result of shape recognition.

Gutierrez-Becker et al. (2017) tackled the aforementioned limitations about description in a multimodal setting by learning correspondences between context-aware Haar-like features from intravascular ultrasound and histology, and inferring displacements by means of a regression forest. Such approach naturally brings the promising tracks related to convolutional neural networks (Greenspan et al., 2016) in the medical domain. The alignment problem could directly benefit from the computational power of such methods for learning correspondences between two different modalities. For example, a method for real-time 2D-3D registration of tools images with 3D CT was developed by Miao et al. (2016). A survey on deep learning in medical image analysis was recently proposed by Litjens et al. (2017).

Part-to-whole. It is not uncommon for histopathology laboratories to receive tissue samples that are (p1) too wide (Fig. 6) or (p2) too thick (Fig. 7) to be processed as they are. The sample is therefore cut into separate sub-blocks, each of which is processed individually. If no scan of each sub-block is available, one has to keep track on which part of 
the sample each sub-block corresponds to and use that knowledge to initialise the registration of histological sections with the clinical image, or manually align them. As for problem (p2), attempts at using similarity measures have been made to initialise registrations, but those are ambiguous and rely on absolute measures rather than relative ones (Yang et al., 2012). On that matter, it was shown that direct comparison of images from different modalities is non-trivial, and fails to reliably determine slice correspondences (Xiao et al., 2011). A solution to (p1) was proposed by Pichat et al. (2017) via a representation of images using their level lines in order to come down to a monomodal problem, and the identification of common elements of shape.

Fusion. It seems that the first true attempt at drawing mutual enrichment from separate modalities (mass spectrometry and microscopy) has been made by Van de Plas et al. (2015). This seems reasonable to think that one goal of combining information could also be to actually do so through fusion (as opposed to only overlaying images), and thereby for example, increase the spatial resolution as well as augment the informative power of 3D medical imaging.

\subsection{Concluding remarks}

The problem of histology reconstruction using 3D medical images — or by association, that of establishing correspondences between histology and 3D medical images - requires at least four experts: a histopathologist, a physicist, a computer scientist and a physician in order to answer a single biological question. Interdisciplinary collaborations are essential and communication on the object to be delivered/handled at every stage, time constraints and resources is critical (what? how? when?). This allows avoiding compromises, thinking backward and instead appropriately (re)defining a problem (Cristancho et al., 2017). For the sake of illustration, such a timeline is presented in Figure 2 of the Supplementary Methods 1 from the work by Hawrylycz et al. (2012). Cross-field awareness is crucial as data analyses rely on the assumption that the object remains similar enough through time and stages to be reliably compared across modalities. Improvements are consistently being made to minimise alterations of tissue and towards understanding the causes and effects of such variations.

Ex vivo MRI is commonly applied in neuroscience for a better understanding of the contrast mechanisms of disease-induced tissue changes. Its use supposes that the tissue has been preserved (fixed/frozen). As a matter of fact, the effect of time and storage conditions on the magnetic properties of post mortem tissue is important for correct interpretation of in vivo clinical results based upon ex vivo measurements.

According to Fischer et al. (1989) and more recently to Kaye et al. (2010), quick deep freezing is a satisfactory method of storage for tissue samples (e.g., brain, heart) which does not affect T1 or T2 significantly. However, this method is not applicable to liver and muscle tissues (Duck, 2013).

In contrast, chemical fixation (aldehydes, and commonly formalin) causes reduction in tissue $\mathrm{T} 1$ and increase in T2 relaxation times for human tissue (Duck, 2013), as well as a significant decrease of mean water diffusivity in e.g., nervous tissue. Little is known about how fixative solutions alter the tissue microstructures responsible for its MRI properties: while some effects may be reversible, others may be irreversible (Purea and Webb, 2006; Shepherd et al., 2009). Conducting such investigations may require the imaging of the fresh specimen (fixative-free) as well as examinations of the effects of different fixation protocols (Shepherd et al., 2009).

Lastly, detailing data acquisition protocols goes beyond the scope of this review but it still seems relevant to stress out the importance of generating standard data. As far as image registration is concerned, it is simply a tool designed to achieve accurate and reproducible correspondences between separate images. However, improving it becomes vain if similar attention is not also directed towards ensuring consistent, quality input data within and across institutions (Lin and Shi, 2015; Milidonis et al., 2015; Traboulsee et al., 2016). Standardising protocols is not easy and immediate, and although the importance of such undertaking is acknowledged by many, so is the amount of work that remains to be done. Furthermore, since quantitative measurements are to be extracted from those images and interpreted by clinicians/physicians, a comparable amount awaits computational imaging scientists dealing with such variations (Madabhushi and Lee, 2016).

\section{Acknowledgements}

The authors would like to thank Dr. Smriti Patodia, from UCL Institute of Neurology (Department of Neuropathology), for her comments on Section 2 and the images used in Figures 2 and 5, as well as Dr. Steven Van de Pavert, Mr. 
Marcello Moccia, and Prof. Olga Ciccarelli from UCL Institute of Neurology (NMR Research Unit), for the images used in Figure 6.

This research was supported by the European Research Council (Starting Grant 677697, project BUNGEE-TOOLS), the EPSRC (EP/H046410/1, EP/J020990/1, EP/K005278), the MRC (MR/J01107X/1), the EU-FP7 project VPHDARE@IT (FP7-ICT-2011-9-601055), the UCL Leonard Wolfson Experimental Neurology Centre (PR/ylr/18575), the Alzheimers society (AS-PG-15-025), the NIHR Biomedical Research Unit (Dementia) at UCL, the National Institute for Health Research University College London Hospitals Biomedical Research Centre (NIHR BRC UCLH/UCL High Impact Initiative).

\section{References}

Absinta, M., Nair, G., Filippi, M., Ray-Chaudhury, A., Reyes-Mantilla, M. I., Pardo, C. A., Reich, D. S., 2014. Postmortem magnetic resonance imaging to guide the pathologic cut. Journal of Neuropathology \& Experimental Neurology 73 (8), 780-788. 22

Adda-Bedia, M., Boudaoud, A., Boué, L., Deboeuf, S., 2010. Statistical distributions in the folding of elastic structures. Journal of Statistical Mechanics: Theory and Experiment 2010 (11), P11027. 35

Adler, D. H., Ittyerah, R., Pluta, J., Pickup, S., Liu, W., Wolk, D. A., Yushkevich, P. A., 2016. Probabilistic atlas of the human hippocampus combining ex vivo mri and histology. In: International Conference on Medical Image Computing and Computer-Assisted Intervention. Springer, pp. 63-71. 24, 34

Adler, D. H., Pluta, J., Kadivar, S., Craige, C., Gee, J. C., Avants, B. B., Yushkevich, P. A., 2014. Histology-derived volumetric annotation of the human hippocampal subfields in postmortem mri. Neuroimage 84, 505-523. 5, 9, 10, 11, 12, 13, 24, 25, 30, 34, 36

Alcantarilla, P. F., Bartoli, A., Davison, A. J., 2012. Kaze features. In: European Conference on Computer Vision. Springer, pp. 214-227. 15, 36

Alcantarilla, P. F., Nuevo, J., Bartoli, A. a., 2013. Fast explicit diffusion for accelerated features in nonlinear scale spaces. British Machine Vision Conference (BMVC). 15

Alegro, M., Amaro-Jr, E., Loring, B., Heinsen, H., Alho, E., Zollei, L., Ushizima, D., Grinberg, L. T., 2016. Multimodal whole brain registration: Mri and high resolution histology. In: Proceedings of the IEEE Conference on Computer Vision and Pattern Recognition Workshops. pp. $194-202$. $9,27,28,31$

Ali, W. S. I., Cohen, F. S., 1998. Registering coronal histological 2-d sections of a rat brain with coronal sections of a 3-d brain atlas using geometric curve invariants and b-spline representation. IEEE Transactions on Medical Imaging 17 (6), 957-966. 5, 16, 17, 23, 33

Alic, L., Haeck, J. C., Bol, K., Klein, S., van Tiel, S. T., Wielepolski, P. A., de Jong, M., Niessen, W. J., Bernsen, M., Veenland, J. F., 2011. Facilitating tumor functional assessment by spatially relating $3 \mathrm{~d}$ tumor histology and in vivo mri: image registration approach. PLoS One 6 (8), e22835. 5, 12, 24, 25, 30, 32

Aljabar, P., Heckemann, R. A., Hammers, A., Hajnal, J. V., Rueckert, D., 2009. Multi-atlas based segmentation of brain images: atlas selection and its effect on accuracy. Neuroimage 46 (3), 726-738. 33

Alpert, N., Bradshaw, J., Kennedy, D., Correia, J., 1990. The principal axes transformation-a method for image registration. J Nucl Med 31, 1717-1723. 19

Amunts, K., Lepage, C., Borgeat, L., Mohlberg, H., Dickscheid, T., Rousseau, M.-É., Bludau, S., Bazin, P.-L., Lewis, L. B., Oros-Peusquens, A.-M., et al., 2013. Bigbrain: an ultrahigh-resolution 3d human brain model. Science 340 (6139), 1472-1475. 3, 28, 33

Amunts, K., Zilles, K., 2015. Architectonic mapping of the human brain beyond brodmann. Neuron 88 (6), 1086-1107. 3

Andersson, M., Groseclose, M. R., Deutch, A. Y., Caprioli, R. M., 2008. Imaging mass spectrometry of proteins and peptides: 3d volume reconstruction. Nature Methods 5 (1), 101-108. 11

Andreasen, A., Drewes, A., Assentoft, J., Larsen, N., 1992. Computer-assisted alignment of standard serial sections without use of artificial landmarks. a practical approach to the utilization of incomplete information in 3-d reconstruction of the hippocampal region. Journal of neuroscience methods 45 (3), 199-207. 18

Andrey, P., Maurin, Y., 2005. Free-d: an integrated environment for three-dimensional reconstruction from serial sections. Journal of neuroscience methods 145 (1), 233-244. 11

Annese, J., 2012. The importance of combining mri and large-scale digital histology in neuroimaging studies of brain connectivity. Mapping the connectome: Multi-level analysis of brain connectivity. 3, 5, 20

Annese, J., Schenker-Ahmed, N. M., Bartsch, H., Maechler, P., Sheh, C., Thomas, N., Kayano, J., Ghatan, A., Bresler, N., Frosch, M. P., et al., 2014 Postmortem examination of patient hms brain based on histological sectioning and digital $3 \mathrm{~d}$ reconstruction. Nature communications 5 . 5

Annese, J., Sforza, D., Dubach, M., Bowden, D., Toga, A., 2006. Postmortem high-resolution 3-dimensional imaging of the primate brain: blockface imaging of perfusion stained tissue. Neuroimage 30 (1), 61-69. 5, 26, 33

Arganda-Carreras, I., Fernández-González, R., Muñoz-Barrutia, A., Ortiz-De-Solorzano, C., 2010. 3d reconstruction of histological sections: Application to mammary gland tissue. Microscopy research and technique 73 (11), 1019-1029. 5, 11, 15, 20, 31

Arganda-Carreras, I., Sorzano, C. O., Kybic, J., Ortiz-de Solorzano, C., 2008. bunwarpj: Consistent and elastic registration in imagej, methods and applications. In: Second ImageJ User \& Developer Conference. 12

Atit, K., Shipley, T. F., Tikoff, B., 2013. Twisting space: are rigid and non-rigid mental transformations separate spatial skills? Cognitive processing $14(2), 163-173.2$

Attneave, F., 1954. Some informational aspects of visual perception. Psychological review 61 (3), 183. 36

Auer, M., Regitnig, P., Holzapfel, G. A., 2005. An automatic nonrigid registration for stained histological sections. IEEE Transactions on Image Processing 14 (4), 475-486. 20

Avants, B. B., Epstein, C. L., Grossman, M., Gee, J. C., 2008. Symmetric diffeomorphic image registration with cross-correlation: evaluating automated labeling of elderly and neurodegenerative brain. Medical image analysis 12 (1), 26-41. 28 
Avants, B. B., Tustison, N., Song, G., 2009. Advanced normalization tools (ants). Insight J 2, 1-35. 12

Avants, B. B., Tustison, N. J., Wu, J., Cook, P. A., Gee, J. C., 2011. An open source multivariate framework for n-tissue segmentation with evaluation on public data. Neuroinformatics 9 (4), 381-400. 10

Axer, M., Amunts, K., Grässel, D., Palm, C., Dammers, J., Axer, H., Pietrzyk, U., Zilles, K., 2011. A novel approach to the human connectome: ultra-high resolution mapping of fiber tracts in the brain. Neuroimage 54 (2), 1091-1101. 5

Badano, A., Revie, C., Casertano, A., Cheng, W.-C., Green, P., Kimpe, T., Krupinski, E., Sisson, C., Skrøvseth, S., Treanor, D., et al., 2015. Consistency and standardization of color in medical imaging: a consensus report. Journal of digital imaging 28 (1), 41-52. 8

Bagci, U., Bai, L., 2010. Automatic best reference slice selection for smooth volume reconstruction of a mouse brain from histological images. IEEE Transactions on Medical imaging 29 (9), 1688-1696. 9, 13, 31

Baheerathan, S., Albregtsen, F., Danielsen, H., 1998. Registration of serial sections of mouse liver cell nuclei. Journal of microscopy 192 (1), $37-53$. 4, 31

Bajcsy, P., LEE, S.-C., Lin, A., Folberg, R., 2006. Three-dimensional volume reconstruction of extracellular matrix proteins in uveal melanoma from fluorescent confocal laser scanning microscope images. Journal of microscopy 221 (1), 30-45. 5

Bancroft, J. D., Gamble, M., 2008. Theory and practice of histological techniques. Elsevier Health Sciences. 4

Bardinet, E., Bhattacharjee, M., Dormont, D., Pidoux, B., Malandain, G., Schüpbach, M., Ayache, N., Cornu, P., Agid, Y., Yelnik, J., 2009. A three-dimensional histological atlas of the human basal ganglia. ii. atlas deformation strategy and evaluation in deep brain stimulation for parkinson disease: Clinical article. Journal of neurosurgery 110 (2), 208-219. 33

Barthel, L. K., Raymond, P. A., 1990. Improved method for obtaining 3-microns cryosections for immunocytochemistry. Journal of Histochemistry \& Cytochemistry 38 (9), 1383-1388. 5

Bautista, P. A., Hashimoto, N., Yagi, Y., et al., 2014. Color standardization in whole slide imaging using a color calibration slide. Journal of pathology informatics $5(1), 4.9$

Bautista, P. A., Yagi, Y., 2015. Staining correction in digital pathology by utilizing a dye amount table. Journal of digital imaging 28 (3), $283-294.9$

Bautista, P. A., Yagi, Y., et al., 2010. Improving the visualization and detection of tissue folds in whole slide images through color enhancement Journal of pathology informatics $1(1), 25.10$

Bay, H., Tuytelaars, T., Van Gool, L., 2006. Surf: Speeded up robust features. In: European conference on computer vision. Springer, pp. $404-417$. 14

Beare, R., Richards, K., Murphy, S., Petrou, S., Reutens, D., 2008. An assessment of methods for aligning two-dimensional microscope sections to create image volumes. Journal of neuroscience methods 170 (2), 332-344. 5, 12, 31

Beis, J. S., Lowe, D. G., 1997. Shape indexing using approximate nearest-neighbour search in high-dimensional spaces. In: Computer Vision and Pattern Recognition, 1997. Proceedings., 1997 IEEE Computer Society Conference on. IEEE, pp. 1000-1006. 16

Bejnordi, B. E., Litjens, G., Timofeeva, N., Otte-Höller, I., Homeyer, A., Karssemeijer, N., van der Laak, J. A., 2016. Stain specific standardization of whole-slide histopathological images. IEEE transactions on medical imaging 35 (2), 404-415. 9

Belanger, L. F., LeBlond, C. P., 1946. A method for locating radioactive elements in tissues by covering histological sections with a photographic emulsion. Endocrinology 39 (1), 8-13. 7

Benetazzo, L., Bizzego, A., De Caro, R., Frigo, G., Guidolin, D., Stecco, C., 2011. 3d reconstruction of the crural and thoracolumbar fasciae. Surgical and radiologic anatomy $33(10), 855-862.12$

Besag, J., 1986. On the statistical analysis of dirty pictures. Journal of the Royal Statistical Society. Series B (Methodological), 259-302. 19

Besl, P. J., McKay, N. D., 1992. Method for registration of 3-d shapes. In: Robotics-DL tentative. International Society for Optics and Photonics, pp. 586-606. 17

Bloch, I., 1996. Information combination operators for data fusion: a comparative review with classification. Systems, Man and Cybernetics, Part A: Systems and Humans, IEEE Transactions on 26 (1), 52-67. 20

Boehler, T., van Straaten, D., Wirtz, S., Peitgen, H.-O., 2011. A robust and extendible framework for medical image registration focused on rapid clinical application deployment. Computers in biology and medicine 41 (6), 340-349. 12, 17

Bonnet, M., Djelloul, M., Tillement, V., Tardivel, C., Mounien, L., Trouslard, J., Troadec, J.-D., Dallaporta, M., 2013. Central nucb2/nesfatin-1expressing neurones belong to the hypothalamic-brainstem circuitry activated by hypoglycaemia. Journal of neuroendocrinology 25 (1), $1-13$. 11

Bookstein, F. L., 1989. Principal warps: Thin-plate splines and the decomposition of deformations. IEEE Transactions on Pattern Analysis \& Machine Intelligence (6), 567-585. 10

Brandt, R., Rohlfing, T., Rybak, J., Krofczik, S., Maye, A., Westerhoff, M., Hege, H.-C., Menzel, R., 2005. Three-dimensional average-shape atlas of the honeybee brain and its applications. Journal of Comparative Neurology 492 (1), 1-19. 19

Braumann, U.-D., Einenkel, J., Horn, L.-C., Kuska, J.-P., Löffler, M., Scherf, N., Wentzensen, N., 2006. Registration of histologic colour images of different staining. In: Bildverarbeitung für die Medizin 2006. Springer, pp. 231-235. 15, 17

Braumann, U.-D., Kuska, J.-P., Einenkel, J., Horn, L.-C., Löffler, M., Höckel, M., 2005. Three-dimensional reconstruction and quantification of cervical carcinoma invasion fronts from histological serial sections. Medical Imaging, IEEE Transactions on 24 (10), 1286-1307. 9, 14, 16, 17, 19,31

Breen, M. S., Lancaster, T. L., Lazebnik, R. S., Nour, S. G., Lewin, J. S., Wilson, D. L., 2003. Three-dimensional method for comparing in vivo interventional $\mathrm{mr}$ images of thermally ablated tissue with tissue response. Journal of Magnetic Resonance Imaging 18 (1), 90-102. 30

Breen, M. S., Lancaster, T. L., Wilson, D. L., 2005a. Correcting spatial distortion in histological images. Computerized Medical Imaging and Graphics 29 (6), 405-417. 10

Breen, M. S., Lazebnik, R. S., Wilson, D. L., 2005b. Three-dimensional registration of magnetic resonance image data to histological sections with model-based evaluation. Annals of biomedical engineering 33 (8), 1100-1112. 5, 10, 26, 29, 31, 32

Brett, M., Johnsrude, I. S., Owen, A. M., 2002. The problem of functional localization in the human brain. Nature reviews neuroscience 3 (3), 243-249. 33

Brey, E. M., King, T. W., Johnston, C., McIntire, L. V., Reece, G. P., Patrick, C. W., 2002. A technique for quantitative three-dimensional analysis of microvascular structure. Microvascular research 63 (3), 279-294. 12 
Bronchti, G., Heil, P., Sadka, R., Hess, A., Scheich, H., Wollberg, Z., 2002. Auditory activation of visualcortical areas in the blind mole rat (spalax ehrenbergi). European Journal of Neuroscience 16 (2), 311-329. 32

Brown, M., Lowe, D. G., 2007. Automatic panoramic image stitching using invariant features. International journal of computer vision 74 (1), 59-73. 12,35

Brown, M., Szeliski, R., Winder, S., 2005. Multi-image matching using multi-scale oriented patches. In: Computer Vision and Pattern Recognition, 2005. CVPR 2005. IEEE Computer Society Conference on. Vol. 1. IEEE, pp. 510-517. 14

Buesa, R. J., 2007. Histology safety: now and then. Annals of diagnostic pathology 11 (5), 334-339. 4

Bürgel, U., Schormann, T., Schleicher, A., Zilles, K., 1999. Mapping of histologically identified long fiber tracts in human cerebral hemispheres to the mri volume of a reference brain: position and spatial variability of the optic radiation. Neuroimage 10 (5), 489-499. 4, 11, 28

Burton, R. A., Plank, G., Schneider, J. E., Grau, V., Ahammer, H., Keeling, S. L., Lee, J., Smith, N. P., Gavaghan, D., Trayanova, N., et al., 2006. Three-dimensional models of individual cardiac histoanatomy: Tools and challenges. Annals of the New York Academy of Sciences 1080 (1), 301-319. 5, 33

Canny, J., 1986. A computational approach to edge detection. IEEE Transactions on pattern analysis and machine intelligence (6), 679-698. 15

Capek, M., Bruza, P., Janacek, J., Karen, P., Kubinova, L., Vagnerova, R., 2009. Volume reconstruction of large tissue specimens from serial physical sections using confocal microscopy and correction of cutting deformations by elastic registration. Microscopy research and technique 72 (2), 110-119. 5, 11

Čapek, M., Janáček, J., Kubínová, L., 2006. Methods for compensation of the light attenuation with depth of images captured by a confocal microscope. Microscopy research and technique 69 (8), 624-635. 11

Cardona, A., Saalfeld, S., Schindelin, J., Arganda-Carreras, I., Preibisch, S., Longair, M., Tomancak, P., Hartenstein, V., Douglas, R. J., 2012. Trakem2 software for neural circuit reconstruction. PloS one 7 (6), e38011. 11

Casasent, D., Psaltis, D., 1976. Position, rotation, and scale invariant optical correlation. Applied optics 15 (7), 1795-1799. 14

Casero, R., Siedlecka, U., Jones, E. S., Gruscheski, L., Gibb, M., Schneider, J. E., Kohl, P., Grau, V., 2017. Transformation diffusion reconstruction of three-dimensional histology volumes from two-dimensional image stacks. Medical image analysis 38, 184-204. 9, 19, 26

Ceritoglu, C., Wang, L., Selemon, L. D., Csernansky, J. G., Miller, M. I., Ratnanather, J. T., 2010. Large deformation diffeomorphic metric mapping registration of reconstructed $3 \mathrm{~d}$ histological section images and in vivo mr images. Frontiers in human neuroscience 4, 43. 9, 24, 25

Chakravarty, M. M., Bertrand, G., Descouteaux, M., Sadikot, A. F., Collins, D. L., 2003. The creation of a brain atlas for image guided neurosurgery using serial histological data. In: International Conference on Medical Image Computing and Computer-Assisted Intervention. Springer, pp. 343-350. 9

Chakravarty, M. M., Bertrand, G., Hodge, C. P., Sadikot, A. F., Collins, D. L., 2006. The creation of a brain atlas for image guided neurosurgery using serial histological data. Neuroimage 30 (2), 359-376. 5, 9, 12, 24, 25, 33

Chappelow, J., Bloch, B. N., Rofsky, N., Genega, E., Lenkinski, R., DeWolf, W., Madabhushi, A., 2011a. Elastic registration of multimodal prostate mri and histology via multiattribute combined mutual information. Medical Physics 38 (4), 2005-2018. 21, 32

Chappelow, J., Tomaszewski, J. E., Feldman, M., Shih, N., Madabhushi, A., 2011b. Histostitcher@: An interactive program for accurate and rapid reconstruction of digitized whole histological sections from tissue fragments. Computerized Medical Imaging and Graphics 35 (7), 557-567. 11, 12

Chen, Q.-s., Defrise, M., Deconinck, F., 1994. Symmetric phase-only matched filtering of fourier-mellin transforms for image registration and recognition. IEEE Transactions on pattern analysis and machine intelligence 16 (12), 1156-1168. 16

Chen, X., Ai, Z., Rasmussen, M., Bajcsy, P., Auvil, L., Welge, M., Leach, L., Vangveeravong, S., Maniotis, A. J., Folberg, R., 2003. Threedimensional reconstruction of extravascular matrix patterns and blood vessels in human uveal melanoma tissue: techniques and preliminary findings. Investigative ophthalmology \& visual science 44 (7), 2834-2840. 4, 13, 31

Chicherova, N., Fundana, K., Müller, B., Cattin, P. C., 2014. Histology to $\mu$ ct data matching using landmarks and a density biased ransac. In: International Conference on Medical Image Computing and Computer-Assisted Intervention. Springer, pp. 243-250. 23

Chklovskii, D. B., Vitaladevuni, S., Scheffer, L. K., 2010. Semi-automated reconstruction of neural circuits using electron microscopy. Current opinion in neurobiology 20 (5), 667-675. 11

Choe, A. S., Gao, Y., Li, X., Compton, K. B., Stepniewska, I., Anderson, A. W., 2011. Accuracy of image registration between mri and light microscopy in the ex vivo brain. Magnetic resonance imaging 29 (5), 683-692. 5, 10, 29, 30

Chow, S. K., Hakozaki, H., Price, D. L., MacLean, N. A., Deerinck, T. J., Bouwer, J. C., Martone, M. E., Peltier, S. T., Ellisman, M. H., 2006. Automated microscopy system for mosaic acquisition and processing. Journal of Microscopy 222 (2), 76-84. 12

Cifor, A., Bai, L., Pitiot, A., 2011. Smoothness-guided 3-d reconstruction of 2-d histological images. Neuroimage 56 (1), 197-211. 13, 18, 30, 31

Cifor, A., Pridmore, T., Pitiot, A., 2009. Smooth 3-d reconstruction for 2-d histological images. In: Information Processing in Medical Imaging. Springer, pp. 350-361. 18

Cohen, F. S., Yang, Z., Huang, Z., Nianov, J., 1998. Automatic matching of homologous histological sections. IEEE transactions on biomedical engineering 45 (5), 642-649. 15, 17

Cohen Fernand, S., Zhaohui, H., Wei, Z., 1995. Invariant matching and identification of curves using b-splines curve representation. IEEE Trans. Image Processing 4 (1), 1-10. 15

Cointepas, Y., Mangin, J.-F., Garnero, L., Poline, J.-B., Benali, H., 2001. Brainvisa: software platform for visualization and analysis of multi-modality brain data. Neuroimage $13(6), 98.11$

Colchester, A., Ourselin, S., Zhu, Y., Bardinet, E., He, Y., Roche, A., Al-Sarraj, S., Nailon, B., Ironside, J., Ayache, N., 2000. 3-d reconstruction of macroscopic optical brain slice images. In: Medical Image Computing and Computer-Assisted Intervention-MICCAI 2000. Springer, pp. 95-105. $13,14,16,30,32$

Collins, D. L., Evans, A. C., 1997. Animal: validation and applications of nonlinear registration-based segmentation. International journal of pattern recognition and artificial intelligence 11 (08), 1271-1294. 25

Collins, D. L., Holmes, C. J., Peters, T. M., Evans, A. C., 1995. Automatic 3-d model-based neuroanatomical segmentation. Human brain mapping 3 (3), 190-208. 12

Collins, D. L., Neelin, P., Peters, T. M., Evans, A. C., 1994. Automatic 3d intersubject registration of mr volumetric data in standardized talairach 
space. Journal of computer assisted tomography 18 (2), 192-205. 12

Coombs, B. D., Rapp, J. H., Ursell, P. C., Reilly, L. M., Saloner, D., 2001. Structure of plaque at carotid bifurcation high-resolution mri with histological correlation. Stroke 32 (11), 2516-2521. 32

Cooper, L., Huang, K., Sharma, A., Mosaliganti, K., Pan, T., 2006. Registration vs. reconstruction: Building 3-d models from 2-d microscopy images. In: Proceedings of the workshop on multiscale biological imaging, data mining and informatics. pp. 57-58. 19

Cooper, L., Sertel, O., Kong, J., Lozanski, G., Huang, K., Gurcan, M., 2009. Feature-based registration of histopathology images with different stains: an application for computerized follicular lymphoma prognosis. Computer methods and programs in biomedicine 96 (3), 182-192. 15, 17

Cornillie, P., Van Den Broeck, W., Simoens, P., 2008. Three-dimensional reconstruction of the remodeling of the systemic vasculature in early pig embryos. Microscopy research and technique 71 (2), 105-111. 11

Cristancho, S., Lingard, L., Forbes, T., Ott, M., Novick, R., 2017. Putting the puzzle together: the role of "problem definition" in complex clinical judgement. Medical education 51 (2), 207-214. 37

Culling, C. F. A., 2013. Handbook of histopathological and histochemical techniques: including museum techniques. Butterworth-Heinemann. 1

Dauguet, J., Bock, D., Reid, R. C., Warfield, S. K., 2007a. Alignment of large image series using cubic b-splines tessellation: Application to transmission electron microscopy data. In: International Conference on Medical Image Computing and Computer-Assisted Intervention. Springer, pp. 710-717. 6

Dauguet, J., Delzescaux, T., Condé, F., Mangin, J.-F., Ayache, N., Hantraye, P., Frouin, V., 2007b. Three-dimensional reconstruction of stained histological slices and 3d non-linear registration with in-vivo mri for whole baboon brain. Journal of neuroscience methods 164 (1), 191-204. 10, 29, 30, 33

Dauguet, J., Mangin, J.-F., Delzescaux, T., Frouin, V., 2004. Robust inter-slice intensity normalization using histogram scale-space analysis. In: International Conference on Medical Image Computing and Computer-Assisted Intervention. Springer, pp. 242-249. 9

Dauguet, J., Peled, S., Berezovskii, V., Delzescaux, T., Warfield, S. K., Born, R., Westin, C.-F., 2007c. Comparison of fiber tracts derived from in-vivo dti tractography with 3d histological neural tract tracer reconstruction on a macaque brain. Neuroimage 37 (2), 530-538. 5, 6

Deboeuf, S., Katzav, E., Boudaoud, A., Bonn, D., Adda-Bedia, M., 2013. Comparative study of crumpling and folding of thin sheets. Physical review letters $110(10), 104301.35$

Delingette, H., Bardinet, E., Rey, D., Lemarechal, J., Montagnat, J., Ourselin, S., Roche, A., Dormont, D., Yelnik, J., Ayache, N., 2001. Yav++: a software platform for medical image processing and visualization. In: Workshop on Interactive Medical Image Visualization and Analysis satellite symposia of MICCAI, IMIVA'01. 29

Delzescaux, T., Dauguet, J., Condé, F., Maroy, R., Frouin, V., 2003. Using 3d non rigid ffd-based method to register post mortem 3d histological data and in vivo mri of a baboon brain. In: Medical Image Computing and Computer-Assisted Intervention-MICCAI 2003. Springer, pp. 965-966. 24,

Demaine, E. D., O'Rourke, J., 2005. A survey of folding and unfolding in computational geometry. Combinatorial and computational geometry 52, 167-211. 35

Dempster, W. T., 1942. The mechanics of paraffin sectioning by the microtome. The Anatomical Record 84 (3), $241-267.35$

Denk, W., Horstmann, H., 2004. Serial block-face scanning electron microscopy to reconstruct three-dimensional tissue nanostructure. PLoS Biol $2(11)$, e329. 6

Deriche, R., 1987. Using canny's criteria to derive a recursively implemented optimal edge detector. International journal of computer vision 1 (2), 167-187. 30

Dezső, K., Papp, V., Bugyik, E., Hegyesi, H., Sáfrány, G., Bödör, C., Nagy, P., Paku, S., 2012. Structural analysis of oval-cell-mediated liver regeneration in rats. Hepatology 56 (4), 1457-1467. 11

Ding, S.-L., Royall, J. J., Sunkin, S. M., Ng, L., Facer, B. A., Lesnar, P., Guillozet-Bongaarts, A., McMurray, B., Szafer, A., Dolbeare, T. A., et al., 2016. Comprehensive cellular-resolution atlas of the adult human brain. Journal of Comparative Neurology 524 (16), 3127-3481. 3

Dorr, A., Lerch, J. P., Spring, S., Kabani, N., Henkelman, R. M., 2008. High resolution three-dimensional brain atlas using an average magnetic resonance image of 40 adult c57bl/6j mice. Neuroimage 42 (1), 60-69. 33

Drew, B., Jones, E. C., Reinsberg, S., Yung, A. C., Goldenberg, S. L., Kozlowski, P., 2010. Device for sectioning prostatectomy specimens to facilitate comparison between histology and in vivo mri. Journal of Magnetic Resonance Imaging 32 (4), 992-996. 22

Dubois, A., Dauguet, J., Herard, A.-S., Besret, L., Duchesnay, E., Frouin, V., Hantraye, P., Bonvento, G., Delzescaux, T., 2007. Automated three-dimensional analysis of histological and autoradiographic rat brain sections: application to an activation study. Journal of Cerebral Blood Flow \& Metabolism 27 (10), 1742-1755. 4, 6, 10, 11, 18, 32

Duck, F. A., 2013. Physical properties of tissues: a comprehensive reference book. Academic press. 37

Edwards, P. J., Nijmeh, A. D., McGurk, M., Odell, E., Fenlon, M. R., Marsden, P. K., Hawkes, D. J., 2005. Validation of pet imaging by alignment to histology slices. In: Medical Image Computing and Computer-Assisted Intervention-MICCAI 2005. Springer, pp. 968-975. 32

Eiben, B., Palm, C., Pietrzyk, U., Amunts, K., Davatzikos, C., 2010. Perspective error correction using registration for blockface volume reconstruction of serial histological sections of the human brain. In: Bildverarbeitung für die Medizin. Citeseer, pp. 301-305. 26

Emmenlauer, M., Ronneberger, O., Ponti, A., Schwarb, P., Griffa, A., Filippi, A., Nitschke, R., Driever, W., Burkhardt, H., 2009. Xuvtools: free, fast and reliable stitching of large 3d datasets. Journal of microscopy 233 (1), 42-60. 12

Falcão, A. X., Udupa, J. K., Samarasekera, S., Sharma, S., Hirsch, B. E., Lotufo, R. d. A., 1998. User-steered image segmentation paradigms: Live wire and live lane. Graphical models and image processing 60 (4), 233-260. 10, 29

Fernandez-Gonzalez, R., Deschamps, T., Idica, A., Malladi, R., de Solorzano, C. O., 2004. Automatic segmentation of histological structures in mammary gland tissue sections. Journal of biomedical optics 9 (3), 444-453. 11

Ferrante, E., Paragios, N., 2017. Slice-to-volume medical image registration: A survey. Medical Image Analysis 39, 101-123. 3, 23

Feuerstein, M., Heibel, H., Gardiazabal, J., Navab, N., Groher, M., 2011. Reconstruction of 3-d histology images by simultaneous deformable registration. In: Medical Image Computing and Computer-Assisted Intervention-MICCAI 2011. Springer, pp. 582-589. 11, 13, 19

Fiala, J. C., 2005. Reconstruct: a free editor for serial section microscopy. Journal of microscopy 218 (1), 52-61. 11

Filippi, M., Rocca, M. A., Barkhof, F., Brück, W., Chen, J. T., Comi, G., DeLuca, G., De Stefano, N., Erickson, B. J., Evangelou, N., et al., 2012. Association between pathological and mri findings in multiple sclerosis. The Lancet Neurology 11 (4), 349-360. 3 
Fischer, B., Modersitzki, J., 2003. Curvature based image registration. Journal of Mathematical Imaging and Vision 18 (1), 81-85. 18, 19

Fischer, H. W., Van Haverbeke, Y., Rinck, P. A., Schmitz-Feuerhake, I., Muller, R. N., 1989. The effect of aging and storage conditions on excised tissues as monitored by longitudinal relaxation dispersion profiles. Magnetic resonance in medicine 9 (3), 315-324. 37

Fischl, B., 2013. Estimating the location of brodmann areas from cortical folding patterns using histology and ex vivo mri. In: Microstructural Parcellation of the Human Cerebral Cortex. Springer, pp. 129-156. 3

Fischler, M. A., Bolles, R. C., 1981. Random sample consensus: a paradigm for model fitting with applications to image analysis and automated cartography. Communications of the ACM 24 (6), 381-395. 16

Fónyad, L., Shinoda, K., Farkash, E. A., Groher, M., Sebastian, D. P., Szász, A. M., Colvin, R. B., Yagi, Y., 2015. 3-dimensional digital reconstruction of the murine coronary system for the evaluation of chronic allograft vasculopathy. Diagnostic pathology 10 (1), 16.11

Frick, A., Möhring, W., Newcombe, N. S., 2014. Development of mental transformation abilities. Trends in cognitive sciences 18 (10), 536-542. 2

Friedrich, F., Beutel, R. G., 2010. The thoracic morphology of nannochorista (nannochoristidae) and its implications for the phylogeny of mecoptera and antliophora. Journal of Zoological systematics and evolutionary Research 48 (1), 50-74. 11

Gaffling, S., Daum, V., Hornegger, J., 2011. Landmark-constrained 3-d histological imaging: A morphology-preserving approach. In: VMV. pp. 309-316. 14, 16, 18, 30, 31

Gaffling, S., Daum, V., Steidl, S., Maier, A., Köstler, H., Hornegger, J., 2015. A gauss-seidel iteration scheme for reference-free 3-d histological image reconstruction. IEEE transactions on medical imaging 34 (2), 514-530. 19, 31

Gaffling, S., Jäger, F., Daum, V., Tauchi, M., Lütjen-Drecoll, E., 2009. Interpolation of histological slices by means of non-rigid registration. In: Bildverarbeitung für die Medizin 2009. Springer, pp. 267-271. 9, 19

Gagnier, K. M., Shipley, T. F., 2013. Completion in the wild: Perception of 3d forms from cross-sections. In: Proceedings of the 35th Annual Meeting of the Cognitive Science Society, Austin, TX. Vol. 31. pp. 2350-2355. 2

Gallagher, A. C., 2012. Jigsaw puzzles with pieces of unknown orientation. In: Computer Vision and Pattern Recognition (CVPR), 2012 IEEE Conference on. IEEE, pp. 382-389. 35

Gangolli, M., Holleran, L., Kim, J. H., Stein, T. D., Alvarez, V., McKee, A. C., Brody, D. L., 2017. Quantitative validation of a nonlinear histology-mri coregistration method using generalized q-sampling imaging in complex human cortical white matter. NeuroImage 153, 152-167. 21, 22, 30, 36

Gareau, D. S., Li, Y., Huang, B., Eastman, Z., Nehal, K. S., Rajadhyaksha, M., 2008. Confocal mosaicing microscopy in mohs skin excisions: feasibility of rapid surgical pathology. Journal of biomedical optics 13 (5), 054001-054001. 11

Gefen, S., Kiryati, N., Nissanov, J., 2008. Atlas-based indexing of brain sections via 2-d to 3-d image registration. Biomedical Engineering, IEEE Transactions on 55 (1), 147-156. 5, 23, 33

Gefen, S., Tretiak, O., Nissanov, J., 2003. Elastic 3-d alignment of rat brain histological images. IEEE transactions on medical imaging 22 (11), 1480-1489. 18

Ghaznavi, F., Evans, A., Madabhushi, A., Feldman, M., 2013. Digital imaging in pathology: whole-slide imaging and beyond. Annual Review of Pathology: Mechanisms of Disease 8, 331-359. 7

Ghorbel, F., 1994. A complete invariant description for gray-level images by the harmonic analysis approach. Pattern recognition letters 15 (10), 1043-1051. 14

Gibson, E., Crukley, C., Gaed, M., Gómez, J. A., Moussa, M., Chin, J. L., Bauman, G. S., Fenster, A., Ward, A. D., 2012. Registration of prostate histology images to ex vivo mr images via strand-shaped fiducials. Journal of Magnetic Resonance Imaging 36 (6), 1402-1412. 5, 16, 23, 30, 32

Gijtenbeek, J. M., Wesseling, P., Maass, C., Burgers, L., van der Laak, J. A., 2006. Three-dimensional reconstruction of tumor microvasculature: simultaneous visualization of multiple components in paraffin-embedded tissue. Angiogenesis 8 (4), 297-305. 12

Goerres, J., Uneri, A., De Silva, T., Ketcha, M., Reaungamornrat, S., Jacobson, M., Vogt, S., Kleinszig, G., Osgood, G., Wolinsky, J.-P., et al., 2017. Spinal pedicle screw planning using deformable atlas registration. Physics in Medicine and Biology 62 (7), 2871. 33

Goldszal, A. F., Tretiak, J., Hand, P. J., Bhasin, S., McEachron, D. L., 1995. Three-dimensional reconstruction of activated columns from 2-[14 c] deoxy-d-glucose data. Neuroimage 2 (1), 9-20. 16

Gonzalez, R. C., 1987. P. wintz digital image processing. Addision-Wesley Publishing Company, 275-281. 11

Gonzalez, R. C., Woods, R. E., 2002. Digital image processing. 9

Gore, J. C., 2015. Biomedical imaging: current and future trends. Physics World. http://medicalphysicsweb.org/cws/article/opinion/ 60791. 3

Goshtasby, A., 1988. Image registration by local approximation methods. Image and Vision Computing 6 (4), 255-261. 22

Goubran, M., Crukley, C., de Ribaupierre, S., Peters, T. M., Khan, A. R., 2013. Image registration of ex-vivo mri to sparsely sectioned histology of hippocampal and neocortical temporal lobe specimens. Neuroimage 83, 770-781. 10, 24, 26, 30, 32,34

Goubran, M., de Ribaupierre, S., Hammond, R. R., Currie, C., Burneo, J. G., Parrent, A. G., Peters, T. M., Khan, A. R., 2015. Registration of in-vivo to ex-vivo mri of surgically resected specimens: A pipeline for histology to in-vivo registration. Journal of neuroscience methods $241,53-65$. 26, 30,32

Graham, L., Orenstein, J. M., 2007. Processing tissue and cells for transmission electron microscopy in diagnostic pathology and research. Nature protocols 2 (10), 2439-2450. 6

Greenspan, H., van Ginneken, B., Summers, R. M., 2016. Guest editorial deep learning in medical imaging: Overview and future promise of an exciting new technique. IEEE Transactions on Medical Imaging 35 (5), 1153-1159. 36

Groen, H. C., Van Walsum, T., Rozie, S., Klein, S., Van Gaalen, K., Gijsen, F. J., Wielopolski, P. A., Van Beusekom, H. M., De Crom, R., Verhagen, H. J., et al., 2010. Three-dimensional registration of histology of human atherosclerotic carotid plaques to in-vivo imaging. Journal of biomechanics 43 (11), 2087-2092. 5, 26, 29, 31, 32

Guest, E., Baldock, R., 1995. Automatic reconstruction of serial sections using the finite element method. Bioimaging 3 (4), 154-167. 16, 17, 30

Guichard, F., Morel, J., Ryan, R., 2004. Contrast invariant image analysis and pdes. preprint. 36

Gurcan, M. N., Boucheron, L. E., Can, A., Madabhushi, A., Rajpoot, N. M., Yener, B., 2009. Histopathological image analysis: a review. Biomedical Engineering, IEEE Reviews in 2, 147-171. 1, 15

Gutierrez-Becker, B., Mateus, D., Peter, L., Navab, N., 2017. Guiding multimodal registration with learned optimization updates. Medical Image Analysis. 36 
Guy, J. R., Sati, P., Leibovitch, E., Jacobson, S., Silva, A. C., Reich, D. S., 2016. Custom fit 3d-printed brain holders for comparison of histology with mri in marmosets. Journal of Neuroscience Methods 257, 55-63. 22

Hammelrath, L., Škokić, S., Khmelinskii, A., Hess, A., van der Knaap, N., Staring, M., Lelieveldt, B. P., Wiedermann, D., Hoehn, M., 2016. Morphological maturation of the mouse brain: An in vivo mri and histology investigation. NeuroImage 125, 144-152. 22

Handschuh, S., Schwaha, T., Metscher, B. D., 2010. Showing their true colors: a practical approach to volume rendering from serial sections. BMC developmental biology $10(1), 41.5$

Haralick, R. M., 1979. Statistical and structural approaches to texture. Proceedings of the IEEE 67 (5), 786-804. 31

Hardy, W. B., 1899. On the structure of cell protoplasm: Part i. the structure produced in a cell by fixative and post-mortem change. the structure of colloidal matter and the mechanism of setting and of coagulation. The Journal of physiology 24 (2), 158. 4

Harkins, K. D., Xu, J., Dula, A. N., Li, K., Valentine, W. M., Gochberg, D. F., Gore, J. C., Does, M. D., 2015. The microstructural correlates of t1 in white matter. Magnetic resonance in medicine. 22

Harteveld, A., Denswil, N., Siero, J., Zwanenburg, J., Vink, A., Pouran, B., Spliet, W., Klomp, D., Luijten, P., Daemen, M., et al., 2016. Quantitative intracranial atherosclerotic plaque characterization at $7 \mathrm{t}$ mri: an ex vivo study with histologic validation. American Journal of Neuroradiology 37 (5), 802-810. 22

Hartkens, T., Rueckert, D., Schnabel, J. A., Hawkes, D. J., Hill, D. L., 2002. Vtk cisg registration toolkit an open source software package for affine and non-rigid registration of single-and multimodal 3d images. In: Bildverarbeitung für die Medizin 2002. Springer, pp. 409-412. 12

Hawrylycz, M. J., Lein, E. S., Guillozet-Bongaarts, A. L., Shen, E. H., Ng, L., Miller, J. A., Van De Lagemaat, L. N., Smith, K. A., Ebbert, A., Riley, Z. L., et al., 2012. An anatomically comprehensive atlas of the adult human brain transcriptome. Nature 489 (7416), 391-399. 3, 37

Heinly, J., Dunn, E., Frahm, J.-M., 2012. Comparative evaluation of binary features. In: Computer Vision-ECCV 2012. Springer, pp. 759-773. 15

Heinrich, M. P., Jenkinson, M., Bhushan, M., Matin, T., Gleeson, F. V., Brady, M., Schnabel, J. A., 2012. Mind: Modality independent neighbourhood descriptor for multi-modal deformable registration. Medical Image Analysis 16 (7), 1423-1435. 36

Hess, A., Lohmann, K., Gundelfinger, E. D., Scheich, H., 1998. A new method for reliable and efficient reconstruction of 3-dimensional images from autoradiographs of brain sections. Journal of neuroscience methods 84 (1), 77-86. 31, 32

Hibbard, L. S., Hawkins, R. A., 1984. Three-dimensional reconstruction of metabolic data from quantitative autoradiography of rat brain. American Journal of Physiology-Endocrinology and Metabolism 247 (3), E412-E419. 32

Hibbard, L. S., Hawkins, R. A., 1988. Objective image alignment for three-dimensional reconstruction of digital autoradiograms. Journal of neuroscience methods 26 (1), 55-74. 32

Hibbard, L. S., McGlone, J. S., Davis, D. W., Hawkins, R. A., 1987. Three-dimensional representation and analysis of brain energy metabolism. Science 236 (4809), 1641-1646. 32

Hill, D. L., Batchelor, P. G., Holden, M., Hawkes, D. J., 2001. Medical image registration. Physics in medicine and biology 46 (3), R1. 13

Holmes, D. P., Crosby, A. J., 2010. Draping films: A wrinkle to fold transition. Physical review letters 105 (3), 038303.10

Hopwood, N., 1999. "giving body" to embryos: Modeling, mechanism, and the microtome in late nineteenth-century anatomy. Isis, 462-496. 2

Hsieh, J.-W., Liao, H.-Y. M., Fan, K.-C., Ko, M.-T., Hung, Y.-P., 1997. Image registration using a new edge-based approach. Computer Vision and Image Understanding 67 (2), 112-130. 14, 16

Hsu, W.-Y., 2011. Analytic differential approach for robust registration of rat brain histological images. Microscopy research and technique 74 (6), 523-530. 14, 16

Hsu, W.-Y., Poon, W.-F., Sun, Y.-N., 2008. Automatic seamless mosaicing of microscopic images: enhancing appearance with colour degradation compensation and wavelet-based blending. Journal of Microscopy 231 (3), 408-418. 11, 14

Hughes, C., Rouvière, O., Mege-Lechevallier, F., Souchon, R., Prost, R., 2013. Robust alignment of prostate histology slices with quantified accuracy. IEEE Transactions on Biomedical Engineering 60 (2), 281-291. 14, 16

Humm, J., Ballon, D., Hu, Y., Ruan, S., Chui, C., Tulipano, P., Erdi, A., Koutcher, J., Zakian, K., Urano, M., et al., 2003. A stereotactic method for the three-dimensional registration of multi-modality biologic images in animals: Nmr, pet, histology, and autoradiography. Medical physics 30 (9), 2303-2314. 6, 24, 25, 32

Humm, J., Macklis, R. M., Lu, X., Yang, Y., Bump, K., Beresford, B., Chin, L., 1995. The spatial accuracy of cellular dose estimates obtained from $3 \mathrm{~d}$ reconstructed serial tissue autoradiographs. Physics in medicine and biology 40 (1), 163. 16

James, A. P., Dasarathy, B. V., 2014. Medical image fusion: A survey of the state of the art. Information Fusion 19, 4-19. 20

Jia, J., Tang, C.-K., 2008. Image stitching using structure deformation. IEEE Transactions on Pattern Analysis and Machine Intelligence 30 (4), 617-631. 12

Jiang, L., Greenwood, T. R., Hove, E. R. A., Chughtai, K., Raman, V., Winnard, P. T., Heeren, R., Artemov, D., Glunde, K., 2013. Combined mr, fluorescence and histology imaging strategy in a human breast tumor xenograft model. NMR in Biomedicine 26 (3), 285-298. 5, 32

Johnson, G. A., Ali-Sharief, A., Badea, A., Brandenburg, J., Cofer, G., Fubara, B., Gewalt, S., Hedlund, L. W., Upchurch, L., 2007. High-throughput morphologic phenotyping of the mouse brain with magnetic resonance histology. Neuroimage 37 (1), 82-89. 33

Johnson, G. A., Badea, A., Brandenburg, J., Cofer, G., Fubara, B., Liu, S., Nissanov, J., 2010. Waxholm space: an image-based reference for coordinating mouse brain research. Neuroimage 53 (2), 365-372. 5, 28, 33

Johnson, G. A., Calabrese, E., Badea, A., Paxinos, G., Watson, C., 2012. A multidimensional magnetic resonance histology atlas of the wistar rat brain. Neuroimage 62 (3), 1848-1856. 28

Ju, T., Warren, J., Carson, J., Bello, M., Kakadiaris, I., Chiu, W., Thaller, C., Eichele, G., 2006. 3d volume reconstruction of a mouse brain from histological sections using warp filtering. Journal of Neuroscience Methods 156 (1), 84-100. 19, 30, 31, 33

Kakar, S., Torbenson, M., Jain, D., Wu, T.-T., Yeh, M., Ferrell, L. D., 2015. Immunohistochemical pitfalls in the diagnosis of hepatocellular adenomas and focal nodular hyperplasia: accurate understanding of diverse staining patterns is essential for diagnosis and risk assessment. Modern Pathology 28 (1), 159-160. 4

Karen, P., Jirkovská, M., Tomori, Z., Demjénová, E., Janáček, J., Kubinova, L., 2003. Three-dimensional computer reconstruction of large tissue volumes based on composing series of high-resolution confocal images by gluemrc and linkmrc software. Microscopy research and technique $62(5), 415-422.11$

Kaye, E. A., Josan, S., Lu, A., Rosenberg, J., Daniel, B. L., Pauly, K. B., 2010. Consistency of signal intensity and t2* in frozen ex vivo heart muscle, 
kidney, and liver tissue. Journal of Magnetic Resonance Imaging 31 (3), 719-724. 37

Khan, A. M., Rajpoot, N., Treanor, D., Magee, D., 2014. A nonlinear mapping approach to stain normalization in digital histopathology images using image-specific color deconvolution. IEEE Transactions on Biomedical Engineering 61 (6), 1729-1738. 9

Khimchenko, A., Deyhle, H., Schulz, G., Schweighauser, G., Hench, J., Chicherova, N., Bikis, C., Hieber, S. E., Müller, B., 2016. Extending two-dimensional histology into the third dimension through conventional micro computed tomography. NeuroImage 139, 26-36. 23

Kilsdonk, I. D., Jonkman, L. E., Klaver, R., van Veluw, S. J., Zwanenburg, J. J., Kuijer, J. P., Pouwels, P. J., Twisk, J. W., Wattjes, M. P., Luijten, P. R., et al., 2016. Increased cortical grey matter lesion detection in multiple sclerosis with $7 \mathrm{t}$ mri: a post-mortem verification study. Brain 139 (5), 1472-1481. 22

Kim, B., Boes, J. L., Frey, K. A., Meyer, C. R., 1997. Mutual information for automated unwarping of rat brain autoradiographs. Neuroimage 5 (1), 31-40. 32

Kim, B., Frey, K. A., Mukhopadhyay, S., Ross, B. D., Meyer, C. R., 1995. Co-registration of mri and autoradiography of rat brain in three-dimensions following automatic reconstruction of $2 d$ data set. In: Computer vision, virtual reality and robotics in medicine. Springer, pp. 262-266. 32

Kim, P., Abkarian, M., Stone, H. A., 2011. Hierarchical folding of elastic membranes under biaxial compressive stress. Nature materials 10 (12), 952-957. 35

Kim, T.-S., Singh, M., Sungkara, W., Zarow, C., Chui, H., 2000. Automatic registration of postmortem brain slices to mri reference volume. Nuclear Science, IEEE Transactions on 47 (4), 1607-1613. 23, 30

Kindle, L., Kakadiaris, I., Ju, T., Carson, J., 2011. A semiautomated approach for artefact removal in serial tissue cryosections. Journal of microscopy 241 (2), 200-206. 10, 12

Klein, S., Staring, M., Murphy, K., Viergever, M. A., Pluim, J. P., 2010. Elastix: a toolbox for intensity-based medical image registration. Medical Imaging, IEEE Transactions on 29 (1), 196-205. 12, 25

Koh, J., Chung, Y. E., Nahm, J. H., Kim, H. Y., Kim, K.-S., Park, Y. N., Kim, M.-J., Choi, J.-Y., 2016. Intrahepatic mass-forming cholangiocarcinoma: prognostic value of preoperative gadoxetic acid-enhanced mri. European radiology 26 (2), 407-416. 22

Kong, W., Kimia, B. B., 2001. On solving 2d and 3d puzzles using curve matching. In: Computer Vision and Pattern Recognition, 2001. CVPR 2001. Proceedings of the 2001 IEEE Computer Society Conference on. Vol. 2. IEEE, pp. II-583. 10

Koshevoy, P., Tasdizen, T., Whitaker, R., 2006. Implementation of an automatic slice-to-slice registration tool. University of Utah, SCI Institute Technical Report UUSCI-2006-018. 14, 16, 17

Kothari, S., Phan, J. H., Wang, M. D., et al., 2013. Eliminating tissue-fold artifacts in histopathological whole-slide images for improved image-based prediction of cancer grade. Journal of pathology informatics 4 (1), 22. 9, 10

Kovačević, N., Henderson, J., Chan, E., Lifshitz, N., Bishop, J., Evans, A., Henkelman, R., Chen, X., 2005. A three-dimensional mri atlas of the mouse brain with estimates of the average and variability. Cerebral cortex 15 (5), 639-645. 33

Kremer, J. R., Mastronarde, D. N., McIntosh, J. R., 1996. Computer visualization of three-dimensional image data using imod. Journal of structural biology $116(1), 71-76.11$

Krinidis, S., Nikou, C., Pitas, I., 2003a. A global energy function for the alignment of serially acquired slices. Information Technology in Biomedicine, IEEE Transactions on 7 (2), 108-113. 13, 19, 31

Krinidis, S., Nikou, C., Pitas, I., 2003b. Reconstruction of serially acquired slices using physics-based modeling. IEEE Transactions on Information Technology in Biomedicine 7 (4), 394-403. 15, 18

Kroon, D.-J., Slump, C. H., 2009. Mri modalitiy transformation in demon registration. In: Biomedical Imaging: From Nano to Macro, 2009. IEEE International Symposium on. IEEE, pp. 963-966. 23

Laissue, P., Reiter, C., Hiesinger, P., Halter, S., Fischbach, K., Stocker, R., 1999. Three-dimensional reconstruction of the antennal lobe in drosophila melanogaster. Journal of Comparative Neurology 405 (4), 543-552. 11

Lazebnik, R. S., Lancaster, T. L., Breen, M. S., Lewin, J. S., Wilson, D. L., 2003. Volume registration using needle paths and point landmarks for evaluation of interventional mri treatments. IEEE transactions on medical imaging 22 (5), 653-660. 29, 30

Le Nobin, J., Rosenkrantz, A. B., Villers, A., Orczyk, C., Deng, F.-M., Melamed, J., Mikheev, A., Rusinek, H., Taneja, S. S., 2015. Image guided focal therapy for magnetic resonance imaging visible prostate cancer: defining a 3-dimensional treatment margin based on magnetic resonance imaging histology co-registration analysis. The Journal of urology 194 (2), 364-370. 5, 11, 32

Lebenberg, J., Hérard, A.-S., Dubois, A., Dauguet, J., Frouin, V., Dhenain, M., Hantraye, P., Delzescaux, T., 2010. Validation of mri-based 3d digital atlas registration with histological and autoradiographic volumes: an anatomofunctional transgenic mouse brain imaging study. Neuroimage 51 (3), 1037-1046. 6, 28, 29, 30, 31, 33

Lee, J. S., Ahn, S.-H., Lee, D. S., Oh, S. H., Kim, C. S., Jeong, J. M., Park, K. S., Chung, J.-K., Lee, M. C., 2005. Voxel-based statistical analysis of cerebral glucose metabolism in the rat cortical deafness model by $3 \mathrm{~d}$ reconstruction of brain from autoradiographic images. European journal of nuclear medicine and molecular imaging 32 (6), 696-701. 10, 13, 18, 32

Leong, F. W., Brady, M., McGee, J. O., 2003. Correction of uneven illumination (vignetting) in digital microscopy images. Journal of clinical pathology $56(8), 619-621.11$

Leutenegger, S., Chli, M., Siegwart, R. Y., 2011. Brisk: Binary robust invariant scalable keypoints. In: 2011 International conference on computer vision. IEEE, pp. 2548-2555. 15

Levinthal, C., Ware, R., 1972. Three dimensional reconstruction from serial sections. 12

Li, C., Xu, C., Gui, C., Fox, M. D., 2005. Level set evolution without re-initialization: a new variational formulation. In: 2005 IEEE Computer Society Conference on Computer Vision and Pattern Recognition (CVPR'05). Vol. 1. IEEE, pp. 430-436. 10

Li, G., Nikolova, S., Bartha, R., 2006. Registration of in vivo magnetic resonance t 1-weighted brain images to triphenyltetrazolium chloride stained sections in small animals. Journal of neuroscience methods 156 (1), 368-375. 22, 32

Li, X., J. H., Mori, S., 2001. Diffeomap. Kennedy Krieger Insitute \& Johns Hopkins University, https : //www .mristudio.org/. 25

Li, X., Yankeelov, T. E., Rosen, G. D., Gore, J. C., Dawant, B. M., 2009. Enhancement of histological volumes through averaging and their use for the analysis of magnetic resonance images. Magnetic resonance imaging 27 (3), 401-416. 5, 9, 10, 24, 25, 30, 31

Lin, F., Shi, J., 2015. Standardization of diagnostic immunohistochemistry. In: Handbook of Practical Immunohistochemistry. Springer, pp. 17-30. 37 
Litjens, G., Kooi, T., Bejnordi, B. E., Setio, A. A. A., Ciompi, F., Ghafoorian, M., van der Laak, J. A., van Ginneken, B., Sánchez, C. I., 2017. A survey on deep learning in medical image analysis. arXiv preprint arXiv:1702.05747. 36

Liu, Y., Sajja, B. R., Uberti, M. G., Gendelman, H. E., Kielian, T., Boska, M. D., 2012. Landmark optimization using local curvature for point-based nonlinear rodent brain image registration. Journal of Biomedical Imaging 2012, 1. 30

Lobachev, O., Ulrich, C., Steiniger, B. S., Wilhelmi, V., Stachniss, V., Guthe, M., 2017. Feature-based multi-resolution registration of immunostained serial sections. Medical Image Analysis 35, 288-302. 14, 16, 17

Lockwood-Estrin, G., Thom, M., Focke, N. K., Symms, M. R., Martinian, L., Sisodiya, S. M., Duncan, J. S., Eriksson, S. H., 2012. Correlating 3t mri and histopathology in patients undergoing epilepsy surgery. Journal of neuroscience methods 205 (1), 182-189. 6, 32

Lohmann, G., 1998. Extracting line representations of sulcal and gyral patterns in mr images of the human brain. IEEE Transactions on Medical Imaging 17 (6), 1040-1048. 30

Lohmann, K., Gundelfinger, E. D., Scheich, H., Grimm, R., Tischmeyer, W., Richter, K., Hess, A., 1998. Brainview: a computer program for reconstruction and interactive visualization of 3d data sets. Journal of neuroscience methods 84 (1), 143-154. 11

Lopez, X. M., D’Andrea, E., Barbot, P., Bridoux, A.-S., Rorive, S., Salmon, I., Debeir, O., Decaestecker, C., 2013. An automated blur detection method for histological whole slide imaging. PloS one 8 (12), e82710. 8

Lopez Gonzalez, M., Foo, S., Holmes, W., Stewart, W., Muir, K., Condon, B., Welch, G., Forbes, K., 2016. Atherosclerotic carotid plaque composition: A $3 \mathrm{t}$ and $7 \mathrm{t}$ mri-histology correlation study. Journal of Neuroimaging. 22

Lopresti, V., Macagno, E., Levinthal, C., 1973. Structure and development of neuronal connections in isogenic organisms: cellular interactions in the development of the optic lamina of daphnia. Proceedings of the National Academy of Sciences 70 (2), $433-437.12$

Lowe, D. G., 1999. Object recognition from local scale-invariant features. In: Computer vision, 1999. The proceedings of the seventh IEEE international conference on. Vol. 2. Ieee, pp. 1150-1157. 14

Ma, B., Zimmermann, T., Rohde, M., Winkelbach, S., He, F., Lindenmaier, W., Dittmar, K. E., 2007. Use of autostitch for automatic stitching of microscope images. Micron 38 (5), 492-499. 12

Ma, Y., Hof, P., Grant, S., Blackband, S., Bennett, R., Slatest, L., McGuigan, M., Benveniste, H., 2005. A three-dimensional digital atlas database of the adult c57bl/6j mouse brain by magnetic resonance microscopy. Neuroscience 135 (4), 1203-1215. 33

Macenko, M., Niethammer, M., Marron, J. S., Borland, D., Woosley, J. T., Guan, X., Schmitt, C., Thomas, N. E., 2009. A method for normalizing histology slides for quantitative analysis. In: ISBI. Vol. 9. pp. 1107-1110. 9

Madabhushi, A., Lee, G., 2016. Image analysis and machine learning in digital pathology: challenges and opportunities. 1, 3, 37

Magee, D., Song, Y., Gilbert, S., Roberts, N., Wijayathunga, N., Wilcox, R., Bulpitt, A., Treanor, D., 2015. Histopathology in 3d: From three-dimensional reconstruction to multi-stain and multi-modal analysis. Journal of pathology informatics 6. 5, 31

Magee, D., Treanor, D., Crellin, D., Shires, M., Smith, K., Mohee, K., Quirke, P., 2009. Colour normalisation in digital histopathology images. In: Proc Optical Tissue Image analysis in Microscopy, Histopathology and Endoscopy (MICCAI Workshop). Vol. 100. Citeseer. 9

Mailly, P., Haber, S. N., Groenewegen, H. J., Deniau, J.-M., 2010. A 3d multi-modal and multi-dimensional digital brain model as a framework for data sharing. Journal of neuroscience methods 194 (1), 56-63. 5, 33

Maintz, J. A., Viergever, M. A., 1998. A survey of medical image registration. Medical image analysis 2 (1), 1-36. 13, 30

Mair, E., Hager, G. D., Burschka, D., Suppa, M., Hirzinger, G., 2010. Adaptive and generic corner detection based on the accelerated segment test. In: European conference on Computer vision. Springer, pp. 183-196. 15

Majka, P., Wójcik, D. K., 2015. Possum-a framework for three-dimensional reconstruction of brain images from serial sections. Neuroinformatics, 1-14. 11, 25, 31

Malandain, G., Bardinet, E., 2003. Intensity compensation within series of images. In: International Conference on Medical Image Computing and Computer-Assisted Intervention. Springer, pp. 41-49. 9, 10

Malandain, G., Bardinet, É., Nelissen, K., Vanduffel, W., 2004. Fusion of autoradiographs with an mr volume using 2-d and 3-d linear transformations. NeuroImage 23 (1), 111-127. 2, 6, 10, 13, 24, 25, 30, 32

Mallat, S., Zhong, S., 1992. Characterization of signals from multiscale edges. IEEE Transactions on pattern analysis and machine intelligence 14 (7), 710-732. 14

Mangin, J.-F., Coulon, O., Frouin, V., 1998. Robust brain segmentation using histogram scale-space analysis and mathematical morphology. In: International Conference on Medical Image Computing and Computer-Assisted Intervention. Springer, pp. 1230-1241. 10

Mansoori, T., Plank, G., Burton, R., Schneider, J., Kohl, P., Gavaghan, D., Grau, V., 2007. An iterative method for registration of high-resolution cardiac histoanatomical and mri images. In: Biomedical Imaging: From Nano to Macro, 2007. ISBI 2007. 4th IEEE International Symposium on. IEEE, pp. 572-575. 31

Markelj, P., Tomaževič, D., Likar, B., Pernuš, F., 2012. A review of 3d/2d registration methods for image-guided interventions. Medical image analysis 16 (3), 642-661. 23

Marr, D., 1982. Vision: A computational investigation into the human representation and processing of visual information, henry holt and co. Inc., New York, NY 2, 4-2. 36

Martel, G., Kiss, S., Gilbert, G., Anne-Archard, N., Richard, H., Moser, T., Laverty, S., 2016. Differences in the vascular tree of the femoral trochlear growth cartilage at osteochondrosis-susceptible sites in foals revealed by swi $3 \mathrm{t}$ mri. Journal of Orthopaedic Research. 22

Mathiisen, T. M., Lehre, K. P., Danbolt, N. C., Ottersen, O. P., 2010. The perivascular astroglial sheath provides a complete covering of the brain microvessels: an electron microscopic 3d reconstruction. Glia 58 (9), 1094-1103. 11

Mattes, D., Haynor, D. R., Vesselle, H., Lewellen, T. K., Eubank, W., 2003. Pet-ct image registration in the chest using free-form deformations. IEEE transactions on medical imaging 22 (1), 120-128. 27

Mazaheri, Y., Bokacheva, L., Kroon, D.-J., Akin, O., Hricak, H., Chamudot, D., Fine, S., Koutcher, J. A., 2010. Semi-automatic deformable registration of prostate $\mathrm{mr}$ images to pathological slices. Journal of Magnetic Resonance Imaging 32 (5), 1149-1157. 5, 21, 22, 31, 32

Mazziotta, J. C., Toga, A. W., Evans, A., Fox, P., Lancaster, J., 1995. A probabilistic atlas of the human brain: theory and rationale for its development the international consortium for brain mapping (icbm). Neuroimage 2 (2PA), 89-101. 3

McCann, M. T., Ozolek, J. A., Castro, C. A., Parvin, B., Kovacevic, J., 2015. Automated histology analysis: Opportunities for signal processing. IEEE Signal Processing Magazine 32 (1), 78. 12 
Mertzanidou, T., Hipwell, J. H., Reis, S., Bejnordi, B. E., Hermsen, M., Dalmis, M., Vreemann, S., Platel, B., van der Laak, J., Karssemeijer, N., et al., 2016. Whole mastectomy volume reconstruction from $2 \mathrm{~d}$ radiographs and its mapping to histology. In: International Workshop on Digital Mammography. Springer, pp. 367-374. 13, 32

Mertzanidou, T., Hipwell, J. H., Reis, S., Hawkes, D. J., Bejnordi, B. E., Dalmis, M., Vreemann, S., Platel, B., der Laak, J., Karssemeijer, N., et al., 2017. 3d volume reconstruction from serial breast specimen radiographs for mapping between histology and 3d whole specimen imaging. Medical Physics. 32

Meyer, C., Ma, B., Kunju, L. P., Davenport, M., Piert, M., 2013. Challenges in accurate registration of 3-d medical imaging and histopathology in primary prostate cancer. European journal of nuclear medicine and molecular imaging 40 (1), 72-78. 22

Meyer, C. R., Moffat, B. A., Kuszpit, K., Bland, P. L., Chenevert, T., Rehemtulla, A., Ross, B., 2006. A methodology for registration of a histological slide and in vivo mri volume based on optimizing mutual information. Molecular imaging 5 (1), 16. 5, 29

Miao, S., Wang, Z. J., Liao, R., 2016. A cnn regression approach for real-time 2d/3d registration. IEEE transactions on medical imaging 35 (5), 1352-1363. 36

Micheva, K. D., Smith, S. J., 2007. Array tomography: a new tool for imaging the molecular architecture and ultrastructure of neural circuits. Neuron 55 (1), 25-36. 11

Mikula, S., Denk, W., 2015. High-resolution whole-brain staining for electron microscopic circuit reconstruction. Nature methods 12 (6), 541-546. 6

Milidonis, X., Marshall, I., Macleod, M. R., Sena, E. S., 2015. Magnetic resonance imaging in experimental stroke and comparison with histology systematic review and meta-analysis. Stroke 46 (3), 843-851. 37

Millman, R. S., Parker, G. D., 1977. Elements of differential geometry. 17

Mishchenko, Y., 2009. Automation of 3d reconstruction of neural tissue from large volume of conventional serial section transmission electron micrographs. Journal of neuroscience methods 176 (2), 276-289. 11

Modat, M., Cash, D. M., Daga, P., Winston, G. P., Duncan, J. S., Ourselin, S., 2014. Global image registration using a symmetric block-matching approach. Journal of Medical Imaging 1 (2), 024003-024003. 12

Modat, M., Ridgway, G. R., Taylor, Z. A., Lehmann, M., Barnes, J., Hawkes, D. J., Fox, N. C., Ourselin, S., 2010. Fast free-form deformation using graphics processing units. Computer methods and programs in biomedicine 98 (3), 278-284. 12

Modersitzki, J., 2003. Numerical methods for image registration. Oxford university press. 20

Modersitzki, J., 2004. Numerical methods for image registration. Oxford University Press on Demand. 17, 19

Mokhtarian, F., Mackworth, A., 1986. Scale-based description and recognition of planar curves and two-dimensional shapes. IEEE transactions on pattern analysis and machine intelligence (1), 34-43. 17

Moré, J. J., 1978. The levenberg-marquardt algorithm: implementation and theory. In: Numerical analysis. Springer, pp. 105-116. 16

Morel, J.-M., Yu, G., 2011. Is sift scale invariant? Inverse Problems and Imaging 5 (1), 115-136. 36

Mori, K., 2016. From macro-scale to micro-scale computational anatomy: a perspective on the next 20 years. 3

Mortensen, E., Morse, B., Barrett, W., Udupa, J., 1992. Adaptive boundary detection using 'live-wire' two-dimensional dynamic programming. IEEE. 10, 29

Mosaliganti, K., Pan, T., Sharp, R., Ridgway, R., Iyengar, S., Gulacy, A., Wenzel, P., de Bruin, A., Machiraju, R., Huang, K., et al., 2006. Registration and 3d visualization of large microscopy images. In: Medical imaging. International Society for Optics and Photonics, pp. 61442V-61442V. 12

Müller, M., Yigitsoy, M., Heibel, H., Navab, N., 2014. Deformable reconstruction of histology sections using structural probability maps. In: Medical Image Computing and Computer-Assisted Intervention-MICCAI 2014. Springer, pp. 122-129. 19, 31

Nagara, K., Roth, H. R., Nakamura, S., Oda, H., Moriya, T., Oda, M., Mori, K., 2017. Micro-ct guided 3d reconstruction of histological images. In: International Workshop on Patch-based Techniques in Medical Imaging. Springer, pp. 93-101. 15, 16, 22, 31

Nakagawa, Y., Sekiya, I., Kondo, S., Tabuchi, T., Ichinose, S., Koga, H., Tsuji, K., Muneta, T., 2016. Relationship between mri t1rho value and histological findings of intact and radially incised menisci in microminipigs. Journal of Magnetic Resonance Imaging 43 (2), $434-445.22$

Nesbit, G. M., Forbes, G. S., Scheithauer, B. W., Okazaki, H., Rodriguez, M., 1991. Multiple sclerosis: histopathologic and mr and/or ct correlation in 37 cases at biopsy and three cases at autopsy. Radiology 180 (2), 467-474. 20

Nikou, C., Heitz, F., Nehlig, A., Namer, I. J., Armspach, J.-P., 2003. A robust statistics-based global energy function for the alignment of serially acquired autoradiographic sections. Journal of neuroscience methods 124 (1), 93-102. 10, 13, 18, 31, 32

Nir, G., Sahebjavaher, R. S., Kozlowski, P., Chang, S. D., Jones, E. C., Goldenberg, S. L., Salcudean, S. E., 2014. Registration of whole-mount histology and volumetric imaging of the prostate using particle filtering. Medical Imaging, IEEE Transactions on 33 (8), 1601-1613. 5, 20, 23, $30,31,32$

Noroozi, M., Favaro, P., 2016. Unsupervised learning of visual representations by solving jigsaw puzzles. In: European Conference on Computer Vision. Springer, pp. 69-84. 35

Nyul, L. G., Udupa, J. K., 1999. On standardizing the mr image intensity scale. image 1081. 9

Oh, S. W., Harris, J. A., Ng, L., Winslow, B., Cain, N., Mihalas, S., Wang, Q., Lau, C., Kuan, L., Henry, A. M., et al., 2014. A mesoscale connectome of the mouse brain. Nature 508 (7495), 207-214. 3

Onozato, M. L., Hammond, S., Merren, M., Yagi, Y., 2013. Evaluation of a completely automated tissue-sectioning machine for paraffin blocks Journal of clinical pathology 66 (2), 151-154. 11

Osechinskiy, S., Kruggel, F., 2010. Slice-to-volume nonrigid registration of histological sections to mr images of the human brain. Anatomy Research International 2011. 5, 23, 24, 30

Ou, Y., Davatzikos, C., 2009. Dramms: deformable registration via attribute matching and mutual-saliency weighting. In: Information Processing in Medical Imaging. Springer, pp. 50-62. 5, 11, 12, 24, 26, 30, 31, 32

Ourselin, S., Bardinet, E., Dormont, D., Malandain, G., Roche, A., Ayache, N., Tande, D., Parain, K., Yelnik, J., 2001a. Fusion of histological sections and mr images: towards the construction of an atlas of the human basal ganglia. In: Medical Image Computing and Computer-Assisted Intervention-MICCAI 2001. Springer, pp. 743-751. 33

Ourselin, S., Roche, A., Prima, S., Ayache, N., 2000. Block matching: A general framework to improve robustness of rigid registration of medical images. In: International Conference on Medical Image Computing And Computer-Assisted Intervention. Springer, pp. 557-566. 18, 19, 20, 28

Ourselin, S., Roche, A., Subsol, G., Pennec, X., Ayache, N., 2001b. Reconstructing a 3d structure from serial histological sections. Image and vision 
computing 19 (1), 25-31. 13, 16, 18

Paikin, G., Tal, A., 2015. Solving multiple square jigsaw puzzles with missing pieces. In: 2015 IEEE Conference on Computer Vision and Pattern Recognition (CVPR). IEEE, pp. 4832-4839. 10

Palm, C., Axer, M., Gräßel, D., Dammers, J., Lindemeyer, J., Zilles, K., Pietrzyk, U., Amunts, K., 2010. Towards ultra-high resolution fibre tract mapping of the human brain-registration of polarised light images and reorientation of fibre vectors. Frontiers in human neuroscience 4, 9. 5, 6, 10,31

Palm, C., Penney, G. P., Crum, W. R., Schnabel, J. A., Pietrzyk, U., Hawkes, D. J., 2008. Fusion of rat brain histology and mri using weighted multi-image mutual information. In: Medical Imaging. International Society for Optics and Photonics, pp. 69140M-69140M. 5

Palokangas, S., Selinummi, J., Yli-Harja, O., 2007. Segmentation of folds in tissue section images. In: 2007 29th Annual International Conference of the IEEE Engineering in Medicine and Biology Society. IEEE, pp. 5641-5644. 10

Park, H., Piert, M. R., Khan, A., Shah, R., Hussain, H., Siddiqui, J., Chenevert, T. L., Meyer, C. R., 2008. Registration methodology for histological sections and in vivo imaging of human prostate. Academic radiology 15 (8), 1027-1039. 29

Paxinos, G., Huang, X.-F., Toga, A. W., 2000. The rhesus monkey brain in stereotaxic coordinates. 30

Peng, T., Wang, L., Bayer, C., Conjeti, S., Baust, M., Navab, N., 2014. Shading correction for whole slide image using low rank and sparse decomposition. In: International Conference on Medical Image Computing and Computer-Assisted Intervention. Springer, pp. 33-40. 11

Penzias, G., Janowczyk, A., Singanamalli, A., Rusu, M., Shih, N., Feldman, M., Stricker, P. D., Delprado, W., Tiwari, S., Böhm, M., et al., 2016. Autostitcher: An automated program for efficient and robust reconstruction of digitized whole histological sections from tissue fragments. Scientific Reports 6, 29906. 12

Pernkopf, F., Bouchaffra, D., 2005. Genetic-based em algorithm for learning gaussian mixture models. IEEE Transactions on Pattern Analysis and Machine Intelligence 27 (8), 1344-1348. 15

Peters, S. R., 2003. The art of embedding tissue for frozen section. part i: a system for precision face down cryoembedding of tissues using freezing temperature-embedding wells. Journal of histotechnology 26 (1), 11-19. 5

Peters, S. R., 2010. A practical guide to frozen section technique. Springer. 6, 7

Pfaff, T., Narain, R., de Joya, J. M., O’Brien, J. F., 2014. Adaptive tearing and cracking of thin sheets. ACM Transactions on Graphics (TOG) 33 (4), 110. 10, 35

Piccinini, F., Bevilacqua, A., Lucarelli, E., 2013a. Automated image mosaics by non-automated light microscopes: the micromos software tool. Journal of microscopy 252 (3), 226-250. 12

Piccinini, F., Bevilacqua, A., Smith, K., Horvath, P., 2013b. Vignetting and photo-bleaching correction in automated fluorescence microscopy from an array of overlapping images. In: 2013 IEEE 10th International Symposium on Biomedical Imaging. IEEE, pp. 464-467. 11

Pichat, J., Iglesias, J. E., Nousias, S., Yousry, T., Ourselin, S., Modat, M., 2017. Part-to-whole registration of histology and mri using shape elements. arXiv preprint arXiv:1708.08117. 37

Pichat, J., Modat, M., Yousry, T., Ourselin, S., 2015. A multi-path approach to histology volume reconstruction. In: Biomedical Imaging (ISBI), 2015 IEEE 12th International Symposium on. IEEE, pp. 1280-1283. 9, 12, 13

Piert, M., Park, H., Khan, A., Siddiqui, J., Hussain, H., Chenevert, T., Wood, D., Johnson, T., Shah, R. B., Meyer, C., 2009. Detection of aggressive primary prostate cancer with 11c-choline pet/ct using multimodality fusion techniques. Journal of Nuclear Medicine 50 (10), 1585-1593. 29

Pitiot, A., Bardinet, E., Thompson, P. M., Malandain, G., 2006. Piecewise affine registration of biological images for volume reconstruction. Medical image analysis $10(3), 465-483.13,19$

Pluim, J. P., Maintz, J. A., Viergever, M. A., 2000. Image registration by maximization of combined mutual information and gradient information. In: International Conference on Medical Image Computing and Computer-Assisted Intervention. Springer, pp. 452-461. 17

Pocivavsek, L., Dellsy, R., Kern, A., Johnson, S., Lin, B., Lee, K. Y. C., Cerda, E., 2008. Stress and fold localization in thin elastic membranes. Science 320 (5878), 912-916. 35

Preibisch, S., Saalfeld, S., Tomancak, P., 2009. Globally optimal stitching of tiled 3d microscopic image acquisitions. Bioinformatics 25 (11), 1463-1465. 12

Prescott, J., Clary, M., Wiet, G., Pan, T., Huang, K., 2006. Automatic registration of large set of microscopic images using high-level features. In: 3rd IEEE International Symposium on Biomedical Imaging: Nano to Macro, 2006. IEEE, pp. 1284-1287. 15, 17

Priester, A., Natarajan, S., Khoshnoodi, P., Margolis, D. J., Raman, S. S., Reiter, R. E., Huang, J., Grundfest, W., Marks, L. S., 2016. Mri underestimation of prostate cancer geometry: Use of patient-specific molds to correlate images with whole-mount pathology. The Journal of Urology. 3

Prima, S., Ourselin, S., Ayache, N., 2002. Computation of the mid-sagittal plane in 3-d brain images. Medical Imaging, IEEE Transactions on 21 (2), 122-138. 29

Purea, A., Webb, A. G., 2006. Reversible and irreversible effects of chemical fixation on the nmr properties of single cells. Magnetic resonance in medicine 56 (4), 927-931. 37

Qiu, X., Pridmore, T., Pitiot, A., 2009. Correcting distorted histology slices for 3d reconstruction. Med Image Underst Anal. 10

Ramsay, J. G., 1962. Interference patterns produced by the superposition of folds of similar type. The Journal of Geology, 466-481. 35

Rangarajan, A., Chui, H., Mjolsness, E., Pappu, S., Davachi, L., Goldman-Rakic, P., Duncan, J., 1997. A robust point-matching algorithm for autoradiograph alignment. Medical Image Analysis 1 (4), 379-398. 15, 16, 18, 32

Rasband, W., Bright, D., 1995. Nih image-a public domain image-processing program for the macintosh. Microbeam Analysis 4 (3), 137-149. 11

Rey Otero, I., Delbracio, M., 2014. Anatomy of the SIFT Method. Image Processing On Line 4, 370-396. 14

Rey-Otero, I., Delbracio, M., 2015. Is repeatability an unbiased criterion for ranking feature detectors? SIAM Journal on Imaging Sciences 8 (4), 2558-2580. 36

Rey-Otero, I., Morel, J.-M., Delbracio, M., 2015. An analysis of the factors affecting keypoint stability in scale-space. Journal of Mathematical Imaging and Vision, 1-19. 36

Reyes-Aldasoro, C. C., 2009. Retrospective shading correction algorithm based on signal envelope estimation. Electronics letters 45 (9), 454.11

Riddle, A., Dean, J., Buser, J. R., Gong, X., Maire, J., Chen, K., Ahmad, T., Cai, V., Nguyen, T., Kroenke, C. D., et al., 2011. Histopathological correlates of magnetic resonance imaging-defined chronic perinatal white matter injury. Annals of neurology 70 (3), 493-507. 12 
Roberts, N., Magee, D., Song, Y., Brabazon, K., Shires, M., Crellin, D., Orsi, N. M., Quirke, R., Quirke, P., Treanor, D., 2012. Toward routine use of $3 \mathrm{~d}$ histopathology as a research tool. The American journal of pathology 180 (5), 1835-1842. 31

Rohde, G. K., Aldroubi, A., Dawant, B. M., 2003. The adaptive bases algorithm for intensity-based nonrigid image registration. Medical Imaging, IEEE Transactions on 22 (11), 1470-1479. 25, 29

Rohlfing, T., 2012. Image similarity and tissue overlaps as surrogates for image registration accuracy: widely used but unreliable. IEEE transactions on medical imaging 31 (2), 153-163. 31

Rohr, K., Stiehl, H. S., Sprengel, R., Beil, W., Buzug, T. M., Weese, J., Kuhn, M., 1996. Point-based elastic registration of medical image data using approximating thin-plate splines. In: Visualization in Biomedical Computing. Springer, pp. 297-306. 28

Rolls, G., 2012. Fixation and fixatives (2)-factors influencing chemical fixation, formaldehyde and glutaraldehyde. Leica Biosystems. http://www.leicabiosystems.com/pathologyleaders/fixation-and-fixatives-2-factors-influencing-chemicalfixation-formaldehyde-and-glutaraldehyde. 4

Rolls, G., et al., 2008. 101 steps to better histology. Melbourne: Leica Microsystems. 7

Rosai, J., 2007. Why microscopy will remain a cornerstone of surgical pathology. Laboratory investigation 87 (5), $403-408.4$

Rousseeuw, P. J., 1984. Least median of squares regression. Journal of the American statistical association 79 (388), 871-880. 18

Rueckert, D., Sonoda, L. I., Hayes, C., Hill, D. L., Leach, M. O., Hawkes, D. J., 1999. Nonrigid registration using free-form deformations: application to breast mr images. Medical Imaging, IEEE Transactions on 18 (8), 712-721. 22, 25, 29

Ruiz, A., Ujaldon, M., Cooper, L., Huang, K., 2009. Non-rigid registration for large sets of microscopic images on graphics processors. Journal of signal processing systems 55 (1-3), 229-250. 15, 20

Rusu, M., Golden, T., Wang, H., Gow, A., Madabhushi, A., 2015. Framework for 3d histologic reconstruction and fusion with in vivo mri: Preliminary results of characterizing pulmonary inflammation in a mouse model. Medical physics 42 (8), 4822-4832. 13, 24, 25, 31

Saalfeld, S., Cardona, A., Hartenstein, V., Tomančák, P., 2010. As-rigid-as-possible mosaicking and serial section registration of large sstem datasets. Bioinformatics 26 (12), i57-i63. 11, 14, 16

Saalfeld, S., Fetter, R., Cardona, A., Tomancak, P., 2012. Elastic volume reconstruction from series of ultra-thin microscopy sections. Nature methods 9 (7), 717-720. 11, 13, 14, 16, 19

Saalfeld, S., Tomancák, P., 2008. Automatic landmark correspondence detection for imagej. In: Proceedings of the ImageJ User and Developer Conference. pp. 128-133. 14

Sakoe, H., Chiba, S., 1978. Dynamic programming algorithm optimization for spoken word recognition. Acoustics, Speech and Signal Processing, IEEE Transactions on $26(1), 43-49.19$

Saleem, K. S., Logothetis, N. K., 2012. A combined MRI and histology atlas of the rhesus monkey brain in stereotaxic coordinates. Academic Press. 33

Samavati, N., McGrath, D. M., Lee, J., van der Kwast, T., Jewett, M., Ménard, C., Brock, K. K., et al., 2011. Biomechanical model-based deformable registration of mri and histopathology for clinical prostatectomy. Journal of pathology informatics 2 (2), 10. 5, 32, 34

Schilling, K. G., Janve, V., Gao, Y., Stepniewska, I., Landman, B. A., Anderson, A. W., 2018. Histological validation of diffusion mri fiber orientation distributions and dispersion. NeuroImage 165, 200-221. 29

Schmitt, O., Modersitzki, J., Heldmann, S., Wirtz, S., Fischer, B., 2007. Image registration of sectioned brains. International Journal of Computer Vision 73 (1), 5-39. 19

Schneider, C. A., Rasband, W. S., Eliceiri, K. W., et al., 2012. Nih image to imagej: 25 years of image analysis. Nat methods 9 (7), 671-675. 11

Schormann, T., Dabringhaus, A., Zilles, K., 1995. Statistics of deformations in histology and application to improved alignment with mri. Medical Imaging, IEEE Transactions on 14 (1), 25-35. 5, 28

Schormann, T., von Matthey, M., Dabringhaus, A., Zilles, K., 1993. Alignment of 3-d brain data sets originating from mr and histology. Bioimaging $1(2), 119-128.28$

Schormann, T., Zilles, K., 1998. Three-dimensional linear and nonlinear transformations: an integration of light microscopical and mri data. Human brain mapping 6 (5-6), 339-347. 4, 27, 28

Schubert, N., Axer, M., Schober, M., Huynh, A.-M., Huysegoms, M., Palomero-Gallagher, N., Bjaalie, J. G., Leergaard, T. B., Kirlangic, M. E., Amunts, K., et al., 2016. 3d reconstructed cyto-, muscarinic m2 receptor, and fiber architecture of the rat brain registered to the waxholm space atlas. Frontiers in neuroanatomy 10. 7

Schwier, M., Böhler, T., Hahn, H. K., Dahmen, U., Dirsch, O., et al., 2013. Registration of histological whole slide images guided by vessel structures Journal of pathology informatics 4 (2), 10. 12, 15, 17, 31

Sederberg, T. W., Parry, S. R., 1986. Free-form deformation of solid geometric models. ACM SIGGRAPH computer graphics 20 (4), $151-160.19$

Seeley, E. H., Wilson, K. J., Yankeelov, T. E., Johnson, R. W., Gore, J. C., Caprioli, R. M., Matrisian, L. M., Sterling, J. A., 2014. Co-registration of multi-modality imaging allows for comprehensive analysis of tumor-induced bone disease. Bone 61, 208-216. 6, 32

Seewann, A., Kooi, E.-J., Roosendaal, S., Pouwels, P., Wattjes, M., Van der Valk, P., Barkhof, F., Polman, C., Geurts, J., 2012. Postmortem verification of ms cortical lesion detection with 3d dir. Neurology 78 (5), 302-308. 3

Shepherd, T. M., Thelwall, P. E., Stanisz, G. J., Blackband, S. J., 2009. Aldehyde fixative solutions alter the water relaxation and diffusion properties of nervous tissue. Magnetic resonance in medicine 62 (1), 26-34. 37

Shi, S.-R., Key, M. E., Kalra, K. L., 1991. Antigen retrieval in formalin-fixed, paraffin-embedded tissues: an enhancement method for immunohistochemical staining based on microwave oven heating of tissue sections. Journal of Histochemistry \& Cytochemistry 39 (6), $741-748$.

Shirley, P., 2001. Color transfer between images. IEEE Corn 21, 34-41. 9

Shojaii, R., Bacopulos, S., Yang, W., Karavardanyan, T., Spyropoulos, D., Raouf, A., Martel, A., Seth, A., 2014. Reconstruction of 3-dimensional histology volume and its application to study mouse mammary glands. JoVE (Journal of Visualized Experiments) (89), e51325-e51325. 15

Shojaii, R., Martel, A. L., 2009. A novel edge point selection method for registration of histology images. In: Optical Tissue Image analysis in Microscopy, Histopathology and Endoscopy.(OPTIMHisE) Workshop, MICCAI. 15, 17

Simonetti, A. W., Elezi, V. A., Farion, R., Malandain, G., Segebarth, C., Rémy, C., Barbier, E. L., 2006. A low temperature embedding and section registration strategy for $3 \mathrm{~d}$ image reconstruction of the rat brain from autoradiographic sections. Journal of neuroscience methods 158 (2), 
242-250. 14

Singh, M., Rajagopalan, A., Kim, T.-S., Hwang, D., Chui, H., Zhang, X.-L., Lee, A.-Y., Zarow, C., 2008. Co-registration of in vivo human mri brain images to postmortem histological microscopic images. International journal of imaging systems and technology 18 (5-6), 325-335. 5, 23, 30, 32

Singh, R. K., Barbosa-Lorenzi, V. C., Lund, F. W., Grosheva, I., Maxfield, F. R., Haka, A. S., 2016. Degradation of aggregated ldl occurs in complex extracellular sub-compartments of the lysosomal synapse. J Cell Sci, jcs-181743. 11

Sled, J. G., Zijdenbos, A. P., Evans, A. C., 1998. A nonparametric method for automatic correction of intensity nonuniformity in mri data. IEEE transactions on medical imaging 17 (1), 87-97. 11

Solomon, J., Vouga, E., Wardetzky, M., Grinspun, E., 2012. Flexible developable surfaces. In: Computer Graphics Forum. Vol. 31. Wiley Online Library, pp. 1567-1576. 10

Song, Y., Treanor, D., Bulpitt, A. J., Magee, D. R., et al., 2013. 3d reconstruction of multiple stained histology images. Journal of pathology informatics 4 (2), 7. 5, 15, 31

Song, Y., Treanor, D., Bulpitt, A. J., Wijayathunga, N., Roberts, N., Wilcox, R., Magee, D. R., 2014. Unsupervised content classification based nonrigid registration of differently stained histology images. IEEE Transactions on Biomedical Engineering 61 (1), 96-108. 15, 17

Sorzano, C. Ó. S., Thévenaz, P., Unser, M., 2005. Elastic registration of biological images using vector-spline regularization. Biomedical Engineering, IEEE Transactions on 52 (4), 652-663. 12

Sotiras, A., Davatzikos, C., Paragios, N., 2013. Deformable medical image registration: A survey. IEEE transactions on medical imaging 32 (7), 1153-1190. 13, 36

Steenbergen, P., Haustermans, K., Lerut, E., Oyen, R., De Wever, L., Van den Bergh, L., Kerkmeijer, L. G., Pameijer, F. A., Veldhuis, W. B., Pos, F. J., et al., 2015. Prostate tumor delineation using multiparametric magnetic resonance imaging: Inter-observer variability and pathology validation. Radiotherapy and Oncology 115 (2), 186-190. 22

Stille, M., Smith, E. J., Crum, W. R., Modo, M., 2013. 3d reconstruction of 2d fluorescence histology images and registration with in vivo mr images: Application in a rodent stroke model. Journal of neuroscience methods 219 (1), 27-40. 5, 6, 10, 12, 24, 25, 32

Streicher, J., Weninger, W. J., Müller, G. B., 1997. External marker-based automatic congruencing: a new method of $3 \mathrm{~d}$ reconstruction from serial sections. The Anatomical Record 248 (4), 583-602. 4

Stüben, K., Trottenberg, U., 1982. Multigrid methods: Fundamental algorithms, model problem analysis and applications. Springer. 28

Sze, G., De Armond, S. J., Brant-Zawadzki, M., Davis, R. L., Norman, D., Newton, T. H., 1986. Foci of mri signal (pseudo lesions) anterior to the frontal horns: histologic correlations of a normal finding. American journal of neuroradiology 7 (3), 381-387. 20

Tan, Y., Hua, J., Dong, M., 2007. Feature curve-guided volume reconstruction from 2d images. In: Biomedical Imaging: From Nano to Macro, 2007. ISBI 2007. 4th IEEE International Symposium on. IEEE, pp. 716-719. 15, 18

Taxy, J. B., 2009. Frozen section and the surgical pathologist: a point of view. Archives of pathology \& laboratory medicine 133 (7), $1135-1138$. 6

Tekalp, A. M., 1995. Digital video processing. Prentice-Hall, Inc. 11

Thevenaz, P., Ruttimann, U. E., Unser, M., 1998. A pyramid approach to subpixel registration based on intensity. Image Processing, IEEE Transactions on 7 (1), 27-41. 11, 12, 28

Thévenaz, P., Unser, M., 2007. User-friendly semiautomated assembly of accurate image mosaics in microscopy. Microscopy research and technique 70 (2), 135-146. 12

Toews, M., Wells, W., 2009. Sift-rank: Ordinal description for invariant feature correspondence. In: Computer Vision and Pattern Recognition, 2009. CVPR 2009. IEEE Conference on. IEEE, pp. 172-177. 14

Toews, M., Zöllei, L., Wells, W. M., 2013. Feature-based alignment of volumetric multi-modal images. In: International Conference on Information Processing in Medical Imaging. Springer, pp. 25-36. 36

Traboulsee, A., Simon, J., Stone, L., Fisher, E., Jones, D., Malhotra, A., Newsome, S., Oh, J., Reich, D., Richert, N., et al., 2016. Revised recommendations of the consortium of ms centers task force for a standardized mri protocol and clinical guidelines for the diagnosis and follow-up of multiple sclerosis. American Journal of Neuroradiology 37 (3), 394-401. 37

Trahearn, N., Epstein, D., Snead, D., Cree, I., Rajpoot, N., 2014. A fast method for approximate registration of whole-slide images of serial sections using local curvature. In: SPIE Medical Imaging. International Society for Optics and Photonics, pp. 90410E-90410E. 17

Trivedi, H., Turkbey, B., Rastinehad, A. R., Benjamin, C. J., Bernardo, M., Pohida, T., Shah, V., Merino, M. J., Wood, B. J., Linehan, W. M., et al., 2012. Use of patient-specific mri-based prostate mold for validation of multiparametric mri in localization of prostate cancer. Urology 79 (1), 233-239. 22

Uberti, M., Liu, Y., Dou, H., Mosley, R. L., Gendelman, H. E., Boska, M., 2009. Registration of in vivo mr to histology of rodent brains using blockface imaging. In: SPIE Medical Imaging. International Society for Optics and Photonics, pp. 726213-726213. 10, 29

Ulrich, C., Lobachev, O., Steiniger, B., Guthe, M., 2014. Imaging the vascular network of the human spleen from immunostained serial sections. In: VCBM. Citeseer, pp. 69-78. 14, 16, 17, 32

Vahadane, A., Peng, T., Sethi, A., Albarqouni, S., Wang, L., Baust, M., Steiger, K., Schlitter, A. M., Esposito, I., Navab, N., 2016. Structure-preserving color normalization and sparse stain separation for histological images. 9

Van de Plas, R., Yang, J., Spraggins, J., Caprioli, R. M., 2015. Image fusion of mass spectrometry and microscopy: a multimodality paradigm for molecular tissue mapping. Nature methods 12 (4), 366-372. 37

Van Der Kolk, A., Zwanenburg, J., Denswil, N., Vink, A., Spliet, W., Daemen, M., Visser, F., Klomp, D., Luijten, P., Hendrikse, J., 2015. Imaging the intracranial atherosclerotic vessel wall using $7 \mathrm{t}$ mri: initial comparison with histopathology. American Journal of Neuroradiology 36 (4), 694-701. 22

Vandenberghe, M. E., Hérard, A.-S., Souedet, N., Sadouni, E., Santin, M. D., Briet, D., Carré, D., Schulz, J., Hantraye, P., Chabrier, P.-E., et al., 2016. High-throughput 3d whole-brain quantitative histopathology in rodents. Scientific reports 6. 28

Vicory, J., Couture, H. D., Thomas, N. E., Borland, D., Marron, J. S., Woosley, J., Niethammer, M., 2015. Appearance normalization of histology slides. Computerized Medical Imaging and Graphics 43, 89-98. 9

Viergever, M. A., Maintz, J. A., Klein, S., Murphy, K., Staring, M., Pluim, J. P., 2016. A survey of medical image registration-under review. Medical Image Analysis. 13

Viola, P., Wells III, W. M., 1997. Alignment by maximization of mutual information. International journal of computer vision 24 (2), 137-154. 29 
Vovk, U., Pernus, F., Likar, B., 2007. A review of methods for correction of intensity inhomogeneity in mri. IEEE transactions on medical imaging 26 (3), 405-421. 11

Wang, C.-W., Chen, H.-C., 2013. Improved image alignment method in application to x-ray images and biological images. Bioinformatics 29 (15), 1879-1887. 14, 16

Wang, C.-W., Gosno, E. B., Li, Y.-S., 2015. Fully automatic and robust 3d registration of serial-section microscopic images. Scientific reports 5. 6, $12,14,16$

Wang, C.-W., Ka, S.-M., Chen, A., 2014. Robust image registration of biological microscopic images. Scientific reports 4. 6, 11 , 14, 16

Wang, Y.-Y., Chang, S.-C., Wu, L.-W., Tsai, S.-T., Sun, Y.-N., 2007. A color-based approach for automated segmentation in tumor tissue classification. In: 2007 29th Annual International Conference of the IEEE Engineering in Medicine and Biology Society. IEEE, pp. 6576-6579. 9

Ward, A. D., Crukley, C., McKenzie, C. A., Montreuil, J., Gibson, E., Romagnoli, C., Gomez, J. A., Moussa, M., Chin, J., Bauman, G., et al., 2012. Prostate: registration of digital histopathologic images to in vivo $\mathrm{mr}$ images acquired by using endorectal receive coil. Radiology 263 (3), 856-864. 5, 30, 32

Weinstein, R. S., Graham, A. R., Richter, L. C., Barker, G. P., Krupinski, E. A., Lopez, A. M., Erps, K. A., Bhattacharyya, A. K., Yagi, Y., Gilbertson, J. R., 2009. Overview of telepathology, virtual microscopy, and whole slide imaging: prospects for the future. Human pathology 40 (8), 1057-1069. 7

Wells, W. M., Viola, P., Atsumi, H., Nakajima, S., Kikinis, R., 1996. Multi-modal volume registration by maximization of mutual information. Medical image analysis $1(1), 35-51.25$

Weninger, W. J., Meng, S., Streicher, J., Müller, G. B., 1998. A new episcopic method for rapid 3-d reconstruction: applications in anatomy and embryology. Anatomy and embryology 197 (5), 341-348. 4

Wentzensen, N., Braumann, U.-D., Einenkel, J., Horn, L.-C., Doeberitz, M. v. K., Löffler, M., Kuska, J.-P., 2007. Combined serial section-based 3d reconstruction of cervical carcinoma invasion using h\&e/p16ink4a/cd3 alternate staining. Cytometry Part A 71 (5), 327-333. 17, 31

Winkler, H., 2007. 3d reconstruction and processing of volumetric data in cryo-electron tomography. Journal of structural biology 157 (1), $126-137$. 11

Wirtz, S., Fischer, B., Modersitzki, J., Schmitt, O., 2004. Superfast elastic registration of histologic images of a whole rat brain for 3d reconstruction. In: Medical Imaging 2004. International Society for Optics and Photonics, pp. 328-334. 19, 30

Wirtz, S., Papenberg, N., Fischer, B., Schmitt, O., 2005. Robust and staining-invariant elastic registration of a series of images from histologic slices. In: Medical imaging. International Society for Optics and Photonics, pp. 1256-1262. 19

Woods, R. P., Grafton, S. T., Holmes, C. J., Cherry, S. R., Mazziotta, J. C., 1998a. Automated image registration: I. general methods and intrasubject, intramodality validation. Journal of computer assisted tomography 22 (1), 139-152. 12, 25

Woods, R. P., Grafton, S. T., Watson, J. D., Sicotte, N. L., Mazziotta, J. C., 1998b. Automated image registration: Ii. intersubject validation of linear and nonlinear models. Journal of computer assisted tomography 22 (1), 153-165. 12

Xiao, G., Bloch, B. N., Chappelow, J., Genega, E. M., Rofsky, N. M., Lenkinski, R. E., Tomaszewski, J., Feldman, M. D., Rosen, M., Madabhushi, A., 2011. Determining histology-mri slice correspondences for defining mri-based disease signatures of prostate cancer. Computerized Medical Imaging and Graphics 35 (7), 568-578. 22, 32, 37

Xu, Y., Pickering, J. G., Nong, Z., Gibson, E., Arpino, J.-M., Yin, H., Ward, A. D., 2015. A method for 3d histopathology reconstruction supporting mouse microvasculature analysis. PloS one 10 (5), e0126817. 15, 20, 32

Xu, Y., Weaver, J. B., Healy, D. M., Lu, J., 1994. Wavelet transform domain filters: a spatially selective noise filtration technique. IEEE transactions on image processing $3(6), 747-758.14$

Yagi, Y., Gilbertson, J. R., 2005. Digital imaging in pathology: the case for standardization. Journal of telemedicine and telecare 11 (3), 109-116. 8

Yang, S., Yang, Z., Fischer, K., Zhong, K., Stadler, J., Godenschweger, F., Steiner, J., Heinze, H.-J., Bernstein, H.-G., Bogerts, B., et al., 2013. Integration of ultra-high field mri and histology for connectome based research of brain disorders. Front Neuroanat 7 (31). 4,9

Yang, Z., Richards, K., Kurniawan, N. D., Petrou, S., Reutens, D. C., 2012. Mri-guided volume reconstruction of mouse brain from histological sections. Journal of neuroscience methods 211 (2), 210-217. 5, 9, 10, 24, 25, 30, 37

Yelnik, J., Bardinet, E., Dormont, D., Malandain, G., Ourselin, S., Tandé, D., Karachi, C., Ayache, N., Cornu, P., Agid, Y., 2007. A three-dimensional, histological and deformable atlas of the human basal ganglia. i. atlas construction based on immunohistochemical and mri data. Neuroimage 34 (2), 618-638. 6, 9, 26, 28, 29, 33

Yigitsoy, M., Navab, N., 2013. Structure propagation for image registration. IEEE transactions on medical imaging 32 (9), 1657-1670. 10, 12

Yoo, T. S., Ackerman, M. J., Lorensen, W. E., Schroeder, W., Chalana, V., Aylward, S., Metaxas, D., Whitaker, R., 2002. Engineering and algorithm design for an image processing api: a technical report on itk-the insight toolkit. Studies in health technology and informatics, 586-592. 12

Yu, W., 2004. Practical anti-vignetting methods for digital cameras. IEEE Transactions on Consumer Electronics 50 (4), 975-983. 11

Yushkevich, P. A., Avants, B. B., Ng, L., Hawrylycz, M., Burstein, P. D., Zhang, H., Gee, J. C., 2006. 3d mouse brain reconstruction from histology using a coarse-to-fine approach. In: Biomedical Image Registration. Springer, pp. 230-237. 6, 10, 13, 24, 25, 33

Yushkevich, P. A., Avants, B. B., Pluta, J., Das, S., Minkoff, D., Mechanic-Hamilton, D., Glynn, S., Pickup, S., Liu, W., Gee, J. C., et al., 2009. A high-resolution computational atlas of the human hippocampus from postmortem magnetic resonance imaging at 9.4 t. Neuroimage 44 (2), 385-398. 3

Yushkevich, P. A., Wisse, L., Adler, D., Ittyerah, R., Pluta, J. B., Robinson, J. L., Schuck, T., Trojanowski, J. Q., Grossman, M., Detre, J. A., et al., 2016. A framework for informing segmentation of in vivo mri with information derived from ex vivo imaging: Application in the medial temporal lobe. In: Engineering in Medicine and Biology Society (EMBC), 2016 IEEE 38th Annual International Conference of the. IEEE, pp. $6014-6017$. $11,24,34$

Zarow, C., Kim, T.-S., Singh, M., Chui, H., 2004. A standardized method for brain-cutting suitable for both stereology and mri-brain co-registration. Journal of neuroscience methods 139 (2), 209-215. 23

Zhan, Y., Ou, Y., Feldman, M., Tomaszeweski, J., Davatzikos, C., Shen, D., 2007. Registering histologic and mr images of prostate for image-based cancer detection. Academic radiology 14 (11), 1367-1381. 5, 32

Zhao, W., Young, T. Y., Ginsberg, M. D., 1993. Registration and three-dimensional reconstruction of autoradiographic images by the disparity analysis method. Medical Imaging, IEEE Transactions on 12 (4), 782-791. 14, 17, 32 
Zhu, S. C., Yuille, A., 1996. Region competition: Unifying snakes, region growing, and bayes/mdl for multiband image segmentation. IEEE transactions on pattern analysis and machine intelligence 18 (9), 884-900. 10

Zitova, B., Flusser, J., 2003. Image registration methods: a survey. Image and vision computing 21 (11), 977-1000. 13 